\title{
Performance Improvements in Software-defined and Virtualized Wireless Networks
}

\author{
by \\ Chengchao Liang, B.Eng., M.Eng \\ A dissertation submitted to the Faculty of Graduate and Postdoctoral Affairs \\ in partial fulfillment of the requirements for the degree of
}

\section{Doctor of Philosophy}

in

Electrical and Computer Engineering

Ottawa-Carleton Institute for Electrical and Computer Engineering

Department of Systems and Computer Engineering

Carleton University

Ottawa, Ontario, Canada

May, 2017

(c) Copyright

Chengchao Liang, 2017 


\section{Abstract}

In this dissertation, we investigate performance improvements in software-defined and virtualized wireless networks with advanced in-network caching and mobile edge computing (MEC).

Wireless network virtualization (WNV), software-defined networking (SDN), MEC and in-network caching are new promising technologies in next generation wireless networks. Traditionally, in-network caching and MEC have been addressed separately from WNV and SDN. However, it is necessary to jointly consider in-network caching and MEC with WNV and SDN together to provide better services in future wireless networks. Therefore, in this dissertation, we propose to integrate WNV and SDN with in-network caching and MEC in order to improve the end-to-end network performance in wireless networks.

We firstly show that jointly considering WNV and in-network caching is necessary and develop an efficient alternating direction method of multipliers (ADMM)-based distributed virtual resource allocation and in-network caching scheme. Secondly, motivated by the experience of user equipment admission control in traditional wireless networks, we propose a novel concept of virtual network (VN) admission control for wireless virtualization. By limiting the number of VNs embedded in the physical network, VN admission control can effectively guarantee the quality of service experienced by users of VNs and maximize the utilization of the physical networks at the same time. Thirdly, we propose to jointly optimize video streaming, bandwidth 
provisioning and caching strategies in software-defined wireless networks (SDWNs) with limited network resources and quality of service (QoE) requirements. We design a novel mechanism to jointly provide proactive caching, bandwidth provisioning and adaptive video streaming. Lastly, in addition to caching, we integrate MEC into the considered SDWN to enhance the video service in next generation wireless networks. We utilize dual-decomposition method and ADMM design a decentralized algorithm to solve the proposed problems. Simulation results are presented to show the effectiveness of the proposed schemes. 
To my dear, Jinhong 


\section{Acknowledgments}

To pen down this section of my dissertation means I have almost arrived at the destination that I had dreamed every night. This exiting and unbelievable journey started when I wrote my first email to my supervisor, Prof. F. Richard Yu. Thus, I would like to begin this section by thanking him. Without his invaluable support and wonderful supervision, this would have been impossible. His technical insight and on-going encouragement have been a constant source of the motivation. The innumerable discussions with him and his ideas on the research projects have been the most dispensable input for my research. His comments and suggestions on my works not only increase the quality of the research but also provide a source of thought for my career. This work is as much his contribution as is mine for the fact that he has always wholeheartedly supported every decision I have made during this wonderful study. He has better prepared me for the world and I will always be indebted to him for anything I achieve in my future life.

I would like to thank Prof. Halim Yanikomeroglu, for his valuable comments and suggestions. I am privileged to have been working with him in the $5 \mathrm{G}$ research team and am grateful for the time and patience that he donated towards teaching me. I also would like to thank Prof. Chadi Assi, Prof. Amiya Nayak, and Prof. Minyi Huang for serving on my dissertation committee. Special thanks go to Dr. Ngoc Dao, Dr. Gamini Senarath and Dr. Hamid Farmanbar, who provided me valuable and constructive suggestions on my works. 
Many thanks to the fellows at Carleton University, including Dr. Yegui Cai, Dr. Zhexiong Wei, Mr. Yanwei Wang, Mr. Zhiyuan Yin, Dr. Gang Liu, Dr. Lei Chen, Dr. Kan Wang and all other students and visitors in Lab AA509.

Besides, I want to thank Prof. Qianbin Chen and Prof. Zhanjun Liu my master thesis advisors, who encouraged me to pursuit a $\mathrm{PhD}$ degree four years ago.

Special thanks also go to friends Haomin and Zhen who give me in Canada a memorable and often breathtakingly beautiful adventure. I also want to express my thanks to Xuesong, Ziyue, Rui, Man, Zicheng, Jiajie, Chang, and any other individual who generously provides friendship in Ottawa to make my life here amazing.

I cannot image how many sacrifices were made by my family members when I spent long hours in the office, when I was absent from countless familial events and failed in my responsibilities to the family. There is no word to express my gratitude to my family, and in particular, my grandmother, my parents and my wife. This achievement would have not been possible without their unconditional love, support and encouragement. Thanks for always believing in me and making things easy just so that I didnt have to worry about anything but my research. At last, I want to say thanks to my grandfather who was an excellent engineer and brought the heart of engineering to me. I believe he can see my achievement even we are not in the same world. This one is for you all. 


\section{Table of Contents}

Abstract $\quad$ ii

Acknowledgments $\quad$ v

Table of Contents vii

List of Tables $\quad$ xi

List of Figures $\quad$ xii

List of Abbreviations $\quad$ xiv

1 Introduction 1

1.1 Background . . . . . . . . . . . . . . . . . . 1

1.1.1 Enhangcing Content Delivery in Wireless Networks . . . . . . 1

1.1.2 Enhangcing Flexibility in Wireless Networks . . . . . . . . . . 3

1.2 Motivations ..................... 4

1.3 Literature Review . . . . . . . . . . . . . . . . . 6

1.3.1 Enhancing Video Streaming with In-network Caching . . . . . 6

1.3.2 In-network Caching and MEC in Wireless Networks . . . . . . 8

1.3.3 Resource Allocation in Virtualized Wireless Networks . . . . . 9

1.3.4 Traffic Engineering in SDWNs . . . . . . . . . . . 9 
1.4 Dissertation Organization and Contributions . . . . . . . . . 10

1.4.1 List of Publications . . . . . . . . . . . . . . . . . . 12

2 Virtual Resource Allocation and Caching $\quad 15$

2.1 Introduction . . . . . . . . . . . . . . . . . 15

2.2 Framework . . . . . . . . . . . . . . . . . 17

2.3 System Model and Problem Statement _ . . . . . . . . . . . . 22

2.3 .1 Network Model . . . . . . . . . . . . . . . . . . . . . . . 23

2.3 .2 Problem Formulation . . . . . . . . . . . . . . . . 28

2.3.3 Problem Transformation . . . . . . . . . . . . . . . . . . . 31

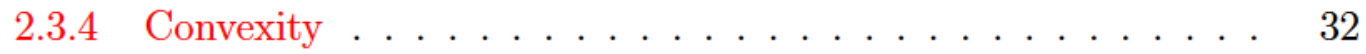

2.4 Virtual Resource Allocation and In-Network Caching via ADMM _. 35

$2.4 .1\left\{\hat{\mathbf{x}}^{j}, \tilde{\mathbf{y}}_{j}, \tilde{\mathbf{z}}_{j}\right\}_{j \in \mathcal{J} \text {-update } \ldots \ldots \ldots \ldots \ldots \ldots \ldots \ldots \ldots \ldots}$

$2.4 .2\{$ x $\}$-update and $\left\{\lambda_{j}\right\}$-update $\ldots \ldots \ldots \ldots \ldots \ldots$

2.4 .3 Stop Criteria and Convergence. . . . . . . . . . . . . . 39

2.4.4 Feasibility, Complexity and Implementations . . . . . . . . 40

2.5 Simulation Results and Discussions _ . . . . . . . . . . . 43

2.5.1 Convergence of the Proposed ADMM-based Scheme . . . . . . 44

2.5.2 The Effects of Virtualization . . . . . . . . . . . . . . . 45

2.5.3 The Effects of Caching with Virtualization . . . . . . . . 48

2.6 Conclusions . . . . . . . . . . . . . . . . . . . . . . 51

3 Robust Admission Control of VNs $\quad 52$

3.1 Introduction . . . . . . . . . . . . . . . . . . . 52

3.2 System Model and Problem Statement _ . . . . . . . . . . . . 54

3.2 .1 Network Model . . . . . . . . . . . . . . . . . . . . . 54

3.2 .2 Problem Statement . . . . . . . . . . . . . . . . . . . . 59 
3.3 Proposed Scheme . . . . . . . . . . . . . . . . . . . . . 61

3.3.1 Robust Admission Control and Resource Leasing . . . . . . 61

3.3.2 Robust Bandwidth Provisioning . . . . . . . . . . . . . 65

3.4 Simulation Results and Discussions _ . . . . . . . . . . . . 68

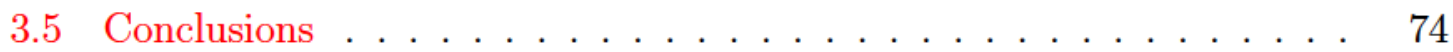

4 Enhancing QoE-aware Caching in SDWNs $\quad 76$

4.1 Introduction . . . . . . . . . . . . . . . . . 76

4.2 System Model and Problem Formulation . . . . . . . . . . . . 79

4.2 .1 Network Model . . . . . . . . . . . . . . . . . . . . . 79

4.2 .2 Problem Formulation . . . . . . . . . . . . . 85

4.3 QoE-aware Joint Bandwidth Provisioning and Caching . . . . . . 88

4.3.1 Proposed Caching Decoupling via Dual Decomposition . . . . 88

4.3.2 Upper Bound Approach to Solving (4.14) . . . . . . . . . . 90

4.3.3 Rounding Methods Based on Marginal Benefits . . . . . . . 93

4.3.4 Computational Complexity, Convergence and Optimality . . . 95

4.3.5 Implementation Design in SDWNs $\ldots \ldots \ldots \ldots$

4.4 Simulation Results and Discussions _ . . . . . . . . . . . . 99

4.4 .1 Algorithm Performance . . . . . . . . . . . . . . . . . . 100

4.4 .2 Network Performance . . . . . . . . . . . . . . . . . . . 101

4.4 .3 Utilization . . . . . . . . . . . . . . . . . . . . . . 104

4.5 Conclusions . . . . . . . . . . . . . . . . . . . 106

5 Enhancing Video Rate Adaptation in SDWNs 112

5.1 Introduction . . . . . . . . . . . . . . . . . . 112

5.2 System Model and Problem Formulation . . . . . . . . . . . . . 114

5.2 .1 Network Model . . . . . . . . . . . . . . . . . . . . . . . . 114 
5.2 .2 Problem Formulation . . . . . . . . . . . . . . . . . . . 119

5.3 Proposed SDN-assisted Video Rate Adaptation with MECC . . . . 122

5.3 .1 Problem Decomposition . . . . . . . . . . . . . . . . 122

5.3.2 Video Rate Adaptation Based on Network Information . . . . 126

5.3.3 Computing Resource Scheduling Based on Network Information 127

5.3.4 ADMM-based Algorithms of Bandwidth Provisioning . . . . 128

5.3 .5 Practical Implementation . . . . . . . . . . . . . . . 131

5.4 Simulation Results and Discussions _. . . . . . . . . . . 133

5.4 .1 Parameters Setup . . . . . . . . . . . . . . . . 133

5.4 .2 Performance Metrics and Schemes . . . . . . . . . . . . 134

5.4 .3 Simulation Results . . . . . . . . . . . . . . . . 135

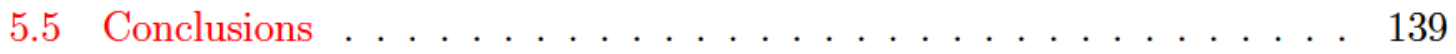

6 Conclusions and Future Works 141

6.1 Conclusions . . . . . . . . . . . . . . . . . . . . . . 141

6.2 Future Works . . . . . . . . . . . . . . . . . . . . . 143

$\begin{array}{ll}\text { List of References } & 145\end{array}$

Appendix A Introduction to ADMM with Consensus Constraint 156

$\begin{array}{ll}\text { Appendix B Computational Complexity Analysis } & 159\end{array}$ 


\section{List of Tables}

2.1 Virtualization scheme in the proposed framework . . . . . . . . 21

2.2 Notations of Chapter $2 . \quad \ldots \ldots \ldots \ldots$

2.3 Algorithm for updating local variables . . . . . . . . . . . . . 38

2.4 Algorithm for virtual resource allocation . . . . . . . . . . . 41

3.1 Notations of Chapter $3 \ldots \ldots \ldots \ldots \ldots$

4.1 Notations of Chapter $4 . \ldots \ldots \ldots$

4.2 Algorithm for the wireless edge caching . . . . . . . . . . . 93

4.3 Algorithm for the proposed joint allocation . . . . . . . . . 95

4.4 Simulation parameters . . . . . . . . . . . . . . . . . . . . . . . 101

4.5 U-vMOS of video resolutions. . . . . . . . . . . . . . . . . . . . 102

5.1 Notations of Chapter $5 . \quad \ldots \ldots \ldots \ldots$

5.2 Algorithm for video rate adaptation and scheduling . . . . . . 126

5.3 Agorithm for bandwidth provisioning . . . . . . . . . . . . . . 129

5.4 Network parameters settings . . . . . . . . . . . . . . . . . . . 133 


\section{List of Figures}

2.1 An example framework of information-centric wireless network virtualization. . . . . . . . . . . . . . . . . . . . . . . . . . 19

2.2 Convergence of ADMM and the effect of $\rho \ldots \ldots \ldots \ldots \ldots$

2.3 Comparison of the incomes of the MVNO . . . . . . . . . 46

2.4 Comparison of the maximum number of satisfied users. . . . . . 47

2.5 The effects of the required data rate. The number of users $=30 \ldots \quad 48$

2.6 Comparison of the backhaul access costs. . . . . . . . . . . . . 49

2.7 Comparison of the average Delay. The number of users $=30 \ldots \ldots \quad 49$

2.8 Comparison of the MVNO utility. . . . . . . . . . . . . 50

3.1 System model of the virtualized wireless network. . . . . . . . . . 54

3.2 The two-stage VN embedding mechanism including admission control, resource borrowing and resource allocation. . . . . . . . . . . 60

3.3 The effect of user arrival rate in VNs on the blocking probability of VNs. 71

3.4 The effect of user arrival rate in VNs on the reserved physical resource. 72

3.5 Performance of different admission control schemes. . . . . . . . . 73

3.6 Effects of varying flows rate on the bandwidth provisioning. . . . . 73

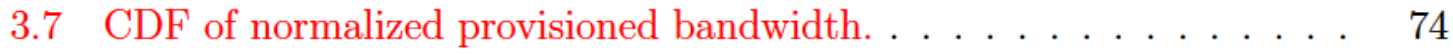

4.1 Network architecture of the cache-enabled SDWN. . . . . . . . . . 79

4.2 The flow diagram in the proposed cache-enabled flow control in SDWNs. 97

4.3 Signaling exchange in cache-enabled SDWNs. . . . . . . . . . 98 
4.4 Convergence and optimality of the proposed scheme. . . . . . . 103

4.5 CDF of round trip delay (backhaul and RAN only) of users. . . . . . 104

4.6 Average mobile network delay with different network setups. . . . . 107

4.7 Average vMoS with different network setups. . . . . . . . . . 108

4.8 Average hit ratio with different network setups. . . . . . . . . . . 109

4.9 Average hitting events with different network setups. . . . . . . . . 110

4.10 Average backhaul load with different network setups. . . . . . . . . 111

5.1 MEC-enabled software-defined mobile network. . . . . . . . . . . 117

5.2 Structure and information exchange of the proposed scheme. . . . . . 131

5.3 Signaling exchange in cache-enabled SDWNs. . . . . . . . . . . . 132

5.4 The mean U-vMoS with different network setups. . . . . . . . 136

5.5 The distribution of video resolutions with different network setups. . 137

5.6 Average load of MEC servers with different network setups. . . . . . 139 


\title{
List of Abbreviations
}

\author{
3GPP 3rd Generation Partnership Project \\ 5G Fifth Generation Mobile Network \\ ABR Adaptive BitRate \\ ADMM Alternating Direction Method of Multipliers \\ BS Base Stations \\ CapEx Capital Expenses \\ CN Core Network \\ CoMP Coordinated Multi-Point operation \\ CSI Channel Status Information \\ DASH Dynamic Adaptive Streaming over HTTP \\ HD High-Definition \\ HetNet Heterogeneous Network \\ HTTP Hypertext Transfer Protocol \\ ICN Information-centric Networking
}




\begin{tabular}{|c|c|}
\hline $\mathrm{InP}$ & Infrastructure Provider \\
\hline LTE & Long Term Evolution \\
\hline MEC & Mobile Edge Computing \\
\hline MECC & Mobile Edge Computing and Caching \\
\hline MIMO & Multiple-Input-Multiple-Output \\
\hline MNO & Mobile Network Operator \\
\hline MOS & Mean Opinion Score \\
\hline MVNO & Mobile Virtual Network Operator \\
\hline NFV & Network Function Virtualization \\
\hline OpEx & Operation Expenses \\
\hline PRB & Physical Resource Block \\
\hline QoE & Quality of Experience \\
\hline QoS & Quality of Service \\
\hline RAN & Radio Access Network \\
\hline $\mathrm{RO}$ & Robust Optimization \\
\hline SBS & Small Base Stations \\
\hline SDMN & Software-Defined Mobile Networks \\
\hline SDN & Software-Defined Networking \\
\hline SINR & Signal to Interference-plus-Noise Ratio \\
\hline
\end{tabular}




$\begin{array}{ll}\text { SP } & \text { Service Provider } \\ \text { TE } & \text { Traffic Engineering } \\ \text { MBS } & \text { Macro Base Stations } \\ \text { U-vMOS } & \text { Unified Video Mean Opinion Score } \\ \text { UE } & \text { User Equipment } \\ \text { VB } & \text { Virtual Bearer } \\ \text { VN } & \text { Virtual Network } \\ \text { VNF } & \text { Virtual Network function } \\ \text { VPN } & \text { Virtual Private Network } \\ \text { WAN } & \text { Wide Area Network } \\ \text { WLAN } & \text { Wireless Local Area Network } \\ \text { WNV } & \text { Wireless Network Virtualization } \\ \text { WVN } & \text { Wireless Virtual Network }\end{array}$




\section{Chapter 1}

\section{Introduction}

\section{$1.1 \quad$ Background}

According to [1], global mobile data traffic will increase nearly eightfold from 2015 to 2020, and 75 percent of them will be video. Thus, the enhancement of content delivery and the improvement of efficient network operation challenge the development of wireless networks (e.g., the fifth generation [5G] mobile networks). To address these challenges, recent advances of information and communications technologies can be explored, such as In-network Caching [2], Wireless Network Virtualization (WNV) [3,4], Software-defined Wireless Networks (SDWNs) [5], and Mobile Edge Computing (MEC) [6].

\subsubsection{Enhangcing Content Delivery in Wireless Networks}

A new technology, information-centric networking (ICN), has attracted great interests from both academia and industry [7-9]. The basic principle behind ICN is to promote content to a first-class citizen in the network. A significant advantage of ICN is to provide native support for scalable and highly efficient content retrieval, 
and meanwhile with enhanced capability for mobility and security. ICN can realize in-network caching to reduce the duplicate content transmissions in networks. A number of research efforts have been dedicated to ICN, including the EU funded projects Publish-Subscribe Internet Technology (PURSUIT) [10] and the US funded projects Named Data Networking (NDN) [11].

Particularly, caching content (e.g., videos) at mobile edge node (e.g., base stations [BSs] and routers) has been proposed as one of the key enablers in next generation mobile networks $[2,12,13]$. The investigation on exploiting caching in future mobile networks has shown that access delays, traffic loads, and network costs can be potentially reduced by caching contents in mobile networks [2].

Meanwhile, to serve as a complement of cloudlet based mobile cloud computing, a new cloud computing paradigm named MEC is proposed and has attracted great interest in 5G recently [6] . Being placed at the edge of radio access networks, the MEC servers can provide sufficient computation resource in physical proximity to users, which guarantees the fulfillment of the demand of fast interactive response by low-latency connections. Moreover, as computational servers are deployed at BSs of the radio access network (RAN), MEC moves computational resources closer to users, which can efficiently improve the quality of services for applications that require intensive computations (e.g., video processing and tracking). Therefore, MEC is envisioned as a promising technique to offer agile and ubiquitous computation augmenting services for mobile devices, by endowing considerable computational capability to mobile communication networks [14]. Moreover, the network can proactively optimize the delivery of content, as RAN-aware content optimization and content caching are two important applications provided by MEC [15]. As the local cache is proposed to be implemented at the MEC server, in-network caching can be integrated with MEC at the mobile edge. 


\subsubsection{Enhangcing Flexibility in Wireless Networks}

Network virtualization has been actively used in Internet research and aims to overcome the resistance of the current Internet to fundamental architecture changes [16-18]. It currently has been considered as one of the most promising technologies for the future Internet [19]. With the tremendous growth in wireless traffic and services, it is natural to extend virtualization to wireless networks. With WNV, mobile network infrastructure can be decoupled from the services that it provides, where differentiated services can coexist on the same infrastructure, maximizing its utilization [4,20-22]. Consequently, multiple wireless virtual networks operated by different SPs can dynamically share the physical substrate wireless networks operated by mobile network operators (MNOs). Since WVN enables the sharing of infrastructure and radio spectrum resources, the capital expenses (CapEx) and the operation expenses $(\mathrm{OpEx})$ of RANs, as well as core networks $(\mathrm{CNs})$, can be reduced significantly. Moreover, Mobile Virtual Network Operator (MVNOs) who may provide some specific telecom services (e.g., VoIP, video call, over-the-top services) can help MNOs attract more users, while MNOs can produce more revenue by leasing the isolated virtualized networks to them and evaluating some new services [23,24]. Meanwhile, WNV provides easier migration to newer products or technologies while supporting legacy products by isolating part of the network [3,23]. In addition, the emerging HetNet need a convergent and powerful network management mechanism, which can be provided by WNV [25].

Software-defined networking (SDN) is an emerging networking paradigm that starts to remove the limitations of current network infrastructures by decoupling the control plane from the data plane [26,27]. In a broader view, SDNs can enable the reduction of complexity and cost of networks, equip programmability into wireless networks, accelerate evolution of networks, and even further catalyze fundamental 
changes in the mobile ecosystem [28-31]. Considering most of data are generating from wireless netwokrs, SDWN has been proposed to support SDN in wireless networks. The successful deployment of SDWNs will depend critically on our ability to jointly provision the backhaul and RANs [32]. Moreover, with the programmability, SDN is considered as a promising candidate to enhance traffic engineering (TE), which is a key component in communication networks [33]. Generally speaking, TE is used to optimize the network and provision services with requirements by directing traffic in networks $[33,34]$.

\subsection{Motivations}

Although some excellent works have been done on WNV and caching, these two important areas have traditionally been addressed separately in the literature. However, as shown in the following, it is necessary to consider these two advanced technologies together to provide better services in next generation wireless networks [35]. Therefore, we jointly study WNV and ICN so as to improve the end-to-end network performance. On one hand, WNV enables the sharing of not only the infrastructure, but also the content, among users from different service providers. Consequently, the CapEx and OpEx of RANs, as well as CNs, can be reduced significantly. On the other hand, as content retrieval (instead of other traditional parameters, such as spectrum efficiency) is put as a high priority in ICN, the processes in WNV (e.g., virtual resource abstracting, slicing, sharing and control) will be significantly affected by ICN. Therefore, jointly considering wireless network virtualization and ICN could improve the end-to-end network performance.

Meanwhile, to effectively guarantee the quality of service (QoS) experienced by users of wireless virtual networks (WVNs) and maximize the utilization of the physical networks, admission control that is very important to embed wireless virtual 
networks has not been studied very well. According to [4], admission control for virtual network is different from traditional user-based admission control. The objective of admission control in wireless virtualization is to maximize the embedded WVNs instead of accessed users. In other word, the network needs to maximize utilization (revenue) while guaranteeing the QoS of existing users by controlling the admission of coming WVNs. In the admission control for WVNs, wireless networks need to conduct relative accurate estimation of physical capacity (e.g., number of users or aggregated throughput) and ensure that the virtual resources allocated to WVNs do not exceed the capacity of underlaying physical networks. However, this is complicated in wireless environment because the number of users, distribution of users and their traffic change dynamically in a particular geographic area, which causes unpredictable aggregated throughput in this area. Obviously, these dynamical properties lead uncertain data and parameters when system optimize the performance. Thus, a suitable solution to cope with these uncertainty is necessary.

The video service is replacing voice and other applications to become the fundamental service in mobile networks [1,36-38]. Moreover, high-definition (HD) videos (e.g., 720p, 1080p, and beyond) that request at least 5-20 Mbps user data rate will be ubiquitous [39], which will bring great challenges to the design and operation of 5G and beyond. As the quality of experience (QoE) of streaming video services mainly includes video resolutions, buffering delays and the frozen (stalling) time [40,41], the SDWN (e.g., provision QoS [42]), in-network caching (e.g., reduce delay [43]) and mobile edge computing (e.g., reduce delay [44], rate adaptive [40]) appear as promising candidates to enhance QoE. However, to the best of our knowledge, QoE-aware joint optimization of the network and cache resources in SDWNs has been largely ignored in the existing research. Unfortunately, the combination of those issues are 
not straightforward, as several challenges are induced by this joint optimization observed as follows. First, bandwidth provisioning in SDWNs should be content and computing-aware, which means it should assign network resources to users based on the caching status and the improvement of the QoE. Second, to enhance the hitting ratio of caches (utilization), caching strategies should be proactive according to the current traffic and resource status, behaviors of users, as well as the requirements of the QoE. Third, since video SDN flows from service providers usually have minimum requirements, the overall QoE performance of the network needs to be guaranteed.

\subsection{Literature Review}

\subsubsection{Enhancing Video Streaming with In-network Caching}

In the line of caching at wireless network, the authors of [45] formulate a delay minimization problem by optimally caching contents at the small BSs (SBSs). This research firstly clusters users with similar content and then deploy a reinforcement learning algorithm to optimize its caching strategy accordingly. [46] proposes a scheme that BSs opportunistically employ Coordinated Multi-Point operation (CoMP) transmission by caching a portion of files so that Multiple-Input-Multiple-Output (MIMO) cooperation gain can be achieved without payload backhaul, while [47] proposes to utilize caching for release part of fronthaul in C-RAN. Unfortunately, the research does not take the real-time mobile network and traffic status into account. In [48], the proposed cache allocation policy considers both the backhaul consumption of SBSs and local storage constraints. [49] proposes a caching selection and BS assignment problem with jointly considering traffic load of radio and backhaul links. However, the cache placement in [49] is static, which is difficult to change dynamically according to users requests. [50] introduces dynamic caching into BS selection from the aspect 
of energy saving. In [51], dynamic caching is proposed to consider the mobility of users, but this research focus more on the cache resource and relationship between nodes. Network resources, such as the backhaul capacity and the spectrum, are not discussed in this research.

Video quality adaptation with radio resource allocation in mobile networks (e.g., especially Long Term Evolution [LTE]) are studied in a number of studies (e.g., [52-56]). A QoE-based evaluation methodology is presented in [52] to assess video capacity of a LTE system for a required target QoE. [53] focuses on video optimization at the base station to maximize the aggregate quality of all the video flows and data flows that it serves. In [54], the authors jointly consider rate allocation and video quality adaptation with time-domain resource partitioning in Heterogeneous Networks (HetNets). Essaili et al. [55] propose a proactive and reactive QoE optimization approaches for adapting the adaptive Hypertext Transfer Protocol (HTTP) video delivery in LTE networks. [56] considers the problem of optimal rate allocation and admission control for adaptive video streaming sessions in wireless networks with user dynamics. Moreover, many studies about video quality adaptation for general networks can be introduced into mobile networks. [57] provides a comprehensive survey on quality of experience of HTTP adaptive streaming.

Joint optimization of video streaming and in-network caching in HetNets can be traced back to [43]. This research suggests that SBSs form a wireless distributed caching network that can efficiently transmit video files to users. Meanwhile, Ahlehagh and Dey [58] take the backhaul and the radio resource into account to realize video-aware caching strategis in RANs for the assurance of maximizing the number of concurrent video sessions. [59], instead of RANs, moves attention of in-network video caching to core networks of LTE. To utilize new technologies in next generation networks, [60] continues the research in specific to the video. [40] conducts a 
comprehensive research that investigates opportunities and challenges of combining the advantages of ABR and RAN caching to increase the video capacity and QoE of wireless networks. Another research combining the caching and video service is [61] where collaborative caching is studied with jointly considering scalable video coding. However, RAN and video quality requirements are not considered.

\subsubsection{In-network Caching and MEC in Wireless Networks}

In [6], an application called RAN-aware content optimization has been proposed as one of the basic service scenarios of MEC. This application can provide an estimated throughput of the wireless link to the video server so that the video rate selection and congestion control can response to the network condition fast. [62] extends this application to which realizes context-aware content localization in order to enhance user QoE in video distribution applications. As video analytics and cache are enabled by MEC, to further utilize the powerful computing resource of MEC server at edge nodes, [40,44,62] propose to jointly combine the advantages of caching and processing to increase the throughput of mobile networks and QoE of users. In [40], the MEC server transcodes a video with higher rate version in the cache to satisfy a request for a lower rate version according to the optimization of the video rate adaptation and the network condition. Along with this research, [44] designs a scheme where multiple MEC caching and servers are collaborative to provide the caching and processing of videos. Compared to those research, our present study introduces TE and SDWN into this joint framework of video caching and processing to enhance the network performance. 


\subsubsection{Resource Allocation in Virtualized Wireless Networks}

Several research projects have been started around the world in the area of wireless network virtualization, such as Environment for Network Innovations (GENI) [63] and Virtualized dIstributed plaTfoRms of smart Objects (VITRO) [64]. The authors of [65] propose a wireless local area network (WLAN) virtualization approach to extend the virtual network embedding from wired networks to wireless networks. Virtualizing eNodeB in 3rd Generation Partnership Project (3GPP) is investigated in [66] from the views of node virtualization and software defined networks.

In addition, some research has been done in resource allocation of virtualized wireless networks, such as virtual resource allocation [67], full-duplex relay scenario [68], scheduling [69] and spectrum sharing [70].

\subsubsection{Traffic Engineering in SDWNs}

TE has been used as a tool to optimize the performance of communication netorks and provision service for a long time [33]. By routing data flows and alloating bandwidth of links to each flow, TE guarantees the QoS requirements of servcies and maximizes the desired network utility. With recent advances of SDN, TE can benefit from the programmability and the flexibility of SDN [71,72]. An optimization of traffic engineering in the SDN controller is formulated by [71] and it shows that the introduction of SDN gets significant improvements in network utilization. The study in [72] tries to leverage SDN to get the enhancement of broadcast communications. The most related studies of our work are TE in SDWN [32,34,42,73]. [34] proposes a multi-path traffic engineering formulation for downlink transmission considering both backhaul and radio access constraints. Moreover, the link buffer status is used as feedback to assist the adjustment of flow allocation. Based on [34], the authors of [42] extend 
TE of SDWNs to real-time video traffic specifically. This research proposes an online method to estimate the effective rate of video flows dynamically. Min flow rate maximization of SDWNs is investigated in [32] with jointly considering TE and physical layer interference management problem by weighted-minimum mean square error algorithm. [73] proposes a weighted cost-minimization problem of TE with jointly considering the traffic load balancing and control-channel setup cost. Compared to those studies, our work exploits TE of SDWN in a MEC and caching-enabled HetNet where content can be retrieved from multiple places.

\subsection{Dissertation Organization and Contributions}

In Chapter 2, we show that jointly considering WNV and ICN is necessary. Specifically, we propose an information-centric wireless network virtualization framework for enabling WNV and ICN. Then, we formulate the virtual resource allocation and in-network caching strategy as an optimization problem, considering not only the revenue earned by serving end users but also the cost of leasing infrastructure. In addition, with recent advances in distributed convex optimization, we develop an efficient alternating direction method of multipliers (ADMM)-based distributed virtual resource allocation and in-network caching scheme. Simulation results are presented to show the effectiveness of the proposed scheme.

In Chapter 3, motivated by the experience of user equipment (UE) admission control in traditional wireless networks, we propose a novel concept of WVN admission control for wireless virtualization. By limiting the number of WVNs embedded in the

physical network, WVN admission control can effectively guarantee QoS experienced by users of WVNs and maximize the utilization of the physical networks at the same time. Specifically, we propose a two-stage WVN embedding mechanism that can decouple short-term physical resource allocation from long-term admission control 
and resource leasing. With recent advances in robust optimization, we formulate the WVN admission control problem as a robust optimization problem. Both the longterm admission control and short-term resource allocation problems are transformed to convex problems, which can be solved efficiently. Simulation results are presented to show the effectiveness of the proposed scheme.

In Chapter 4, we propose to enhance the QoE-aware wireless edge caching with bandwidth provisioning in SDWNs. Specifically, we design a novel mechanism to jointly provide proactive caching, bandwidth provisioning and adaptive video streaming. The caches are requested to retrieve data in advance dynamically according to the behaviors of users, the current traffic and the resource status. Then, we formulate a novel optimization problem regarding the QoE-aware bandwidth provisioning in SDWNs with jointly considering in-network caching strategy. The caching problem is decoupled from the bandwidth provisioning problem by deploying the dualdecomposition method. Additionally, we relax the binary variables to real numbers so that those two problems are formulated as a linear problem and a convex problem, respectively, which can be solved efficiently. Moreover, a practical implementation of SDWNs is proposed, and the corresponding messages exchange of the control plan in SDWNs is designed. Simulation results are presented to show that the latency is decreased and the utilization of caches is improved in the proposed scheme.

In Chapter 5, we jointly consider SDWNs, in-network caching, and MEC to enhance the video service in next generation mobile networks. We use a novel video experience evaluation standard called Unified Video Mean Opinion Score (U-vMOS), which is a more advanced measurement of the video quality based on the well-known video mean opinion score (MOS). With the objective of maximizing the mean UvMOS, an optimization problem is formulated. Due to the coupling of video data rate, computing resource, and traffic engineering (bandwidth provisioning and paths 
selection), the problem becomes intractable in practice. Thus, we utilize dualdecomposition method to decouple those three sets of variables. By this decoupling, video rate adaptation is performed at users with network assistants. End nodes are able to schedule computing resource independently. Traffic engineering is performed by the SDN controller and BSs. Furthermore, to address the challenges of dynamic change of network status and the drawbacks caused by the frequent exchange of information, we design a decentralized algorithm based on ADMM to solve the traffic engineering problem. Extensive simulations are conducted with different system configurations to show the effectiveness of the proposed scheme.

We conclude this study and give related future works in Chapter 6 .

\subsubsection{List of Publications}

\section{Journals}

C. Liang, Y. He, F. Richard Yu, N. Zhao, "Enhancing Video Rate Adaptation with Mobile Edge Computing and Caching in Software-defined Mobile Networks," submitted to IEEE Trans. Multimedia, Feb. 2017. (Chapter 5)

C. Liang, F. Richard Yu, "Enhancing QoE-aware Wireless Edge Caching with Software-defined Wireless Networks," (major revision) submitted to IEEE Trans. Wireless Comm., Dec. 2016. (Chapter 4)

C. Liang, F. Richard Yu, H. Yao, and Z. Han, "Virtual Resource Allocation in Information-Centric Wireless Virtual Networks," IEEE Trans. Veh. Tech., vol. 65, no. 12, pp. 9902-9914, Dec. 2016. (Chapter 2)

C. Liang, F. Richard Yu, and X. Zhang, "Information-Centric Network Function Virtualization Over 5G Mobile Wireless Networks," IEEE Network, vol. 29, no. 3, pp. 68-74, May 2015. (Chapters 1, 2, 3, 4, 5)

C. Liang and F. Richard Yu, "Wireless Network Virtualization: A Survey, Some 
Research Issues and Challenges," IEEE Comm. Surveys and Tutorials, vol. 17, no. 1, pp. 358-380, Firstquarter 2015. (Chapters 1, 2, 3)

C. Liang and F. Richard Yu, "Wireless Virtualization for Next Generation Mobile Cellular Networks," IEEE Wireless Comm., vol. 22, no. 1, pp. 61-69, Feb. 2015. (Chapters 1, 2,3)

\section{Conferences}

C. Liang and F. Richard Yu, "Enhancing Mobile Edge Caching with Bandwidth Provisioning in Software-defined Mobile Networks," accepted for publication in Proc. IEEE ICC'17, Paris, France, May 2017. (Chapters 4)

C. Liang, Y. He, F. Richard Yu, and N. Zhao,, "Energy-Efficient Resource Allocation in Software-Defined Mobile Networks with Mobile Edge Computing and Caching," accepted for publication in IEEE Infocom'17 Workshops, Atlanta, GA, USA, Apr. 2017. (Chapter 5)

C. Liang, F. Richard Yu, "Bandwidth Provisioning in Cache-enabled Softwaredefined Mobile Networks: A Robust Optimization Approach," in Proc. IEEE VTC'16-Fall, Montreal, Canada, Sept. 2016. (Chapter 3)

C. Liang and F. Richard Yu, "Mobile Virtual Network Admission Control and Resource Allocation for Wireless Network Virtualization: A Robust Optimization Approach," in Proc. IEEE Globecom'15, San Diego, CA, Dec. 2015. (Chapter 3)

C. Liang and F. Richard Yu, "Virtual Resource Allocation in Information-Centric Wireless Virtual Networks," in Proc. IEEE ICC'15, London, UK, June 2015. (Chapter 2)

C. Liang and F. Richard Yu, "Distributed Resource Allocation in Virtualized Wireless Cellular Networks based on ADMM," in Proc. IEEE Infocom'15 Workshops, HongKong, P.R. China, Apr. 2015. (Chapter 2)

\section{Patent}


C. Liang, F. Richard Yu, N. Dao, S. Gamini and H. Farmanbar, "Data prefetching in mobile networks", Filed by Huawei, Canada, US provisional patent application no: 85194217US01, Nov. 2016 


\section{Chapter 2}

\section{Virtual Resource Allocation and Caching}

\section{$2.1 \quad$ Introduction}

With virtualization, physical cellular network infrastructure resources and physical radio resources can be abstracted and sliced into virtual cellular network resources holding certain corresponding functionalities, and shared by multiple parties through isolating each other. In other words, virtualizing mobile cellular networks is the process of abstracting, slicing, isolating and sharing the physical resources in mobile cellular networks. Generally speaking, the physical resources in cellular networks are comprised of radio spectrum resource and infrastructure resources, including RANs, CNs, etc.

Another new technology, ICN, has attracted great interests from both academia and industry [7-9]. The basic principle behind ICN is to promote content to a firstclass citizen in the network. A significant advantage of ICN is to provide native support for scalable and highly efficient content retrieval, and meanwhile with enhanced capability for mobility and security. ICN can realize in-network caching to reduce the duplicate content transmissions in networks. 
Although some excellent works have been done on WNV and ICN, these two important areas have traditionally been addressed separately in the literature. However, as shown in the following, it is necessary to consider these two advanced technologies together to provide better services in next generation wireless networks [35]. Therefore, we jointly study WNV and ICN so as to improve the end-to-end network performance. On one hand, wireless network virtualization enables the sharing of not only the infrastructure, but also the content, among users from different service providers. Consequently, the CapEx and OpEx of wireless access networks, as well as core networks, can be reduced significantly. On the other hand, as content retrieval (instead of other traditional parameters, such as spectrum efficiency) is put as a high priority in ICN, the processes in WNV (e.g., virtual resource abstracting, slicing, sharing and control) will be significantly affected by ICN. Therefore, jointly considering WNV and ICN is able to improve the end-to-end network performance. The distinctive features of this chapter are as follows.

- We propose an information-centric wireless network virtualization framework for enabling both WNV and ICN in next generation wireless mobile cellular networks.

- We formulate the virtual resource allocation and in-network caching strategy as an optimization problem, which maximizes the utility of MVNOs, considering not only the revenue earned by serving end users but also the cost of leasing infrastructure.

- The formulated problem has high computational complexity, and the centralized solution may suffer from signaling overhead, outdated dynamics information, and scalability issues. Therefore, with recent advances in distributed convex optimization [74], we develop an efficient ADMM-based distributed virtual resource allocation and in-network caching scheme. 
- Extensive simulations are conducted with different system configurations to show the effectiveness of the proposed scheme. It is shown that we can take the advantages of both WNV and in-network caching with the proposed schemes.

The rest of this chapter is organized as follows. The proposed information-centric wireless network virtualization framework is presented in Section 2.2. Section 2.3 introduces the system model and formulates the proposed problem. Section 2.4 presents two proposed algorithms solved the proposed problem and the corresponding analysis. Simulation results are discussed in Section 2.5. Finally, we conclude this chapter in Section 2.6.

\subsection{Framework}

Due to the trend of decoupling network services (functions) from specific network hardware, more flexible networking is preferred in future mobile network frameworks (e.g., SDN). Unlike the current physical networks (e.g., cellular and core networks) that are designed for all different services, a specific virtual network (VN) operated by one MVNO may be requested by only one service type (e.g., streaming video or online game). Thus, it is important to consider the performance of MVNOs. In this chapter, we adopt the business model comprised of three roles, Infrastructure Providers (InPs), MVNOs, Service Providers (SPs), given in [24] explained as follows:

- InPs operate several physical mobile networks. Each InP has their own RAN including amount of macro base stations (MBSs) and SBSs to cover a certain area. These physical mobile networks can be shared with each other, which means a wireless virtual network (WVN) can be embedded into networks belonged to different InPs.

- $M V N O$ leases physical mobile networks from InPs so that MVNs can be created, 
embedded and operated. Moreover, we assume the MVNO can lease physical resources from any InPs through paying fees, because network sharing has been considered in next generation mobile networks.

- $S P s$ request MVNs from MVNOs and directly provide services (e.g., real time video and online game) to end users.

Traditionally, dedicated physical resources from specific operators are used for content delivery. As these physical resources cannot be shared by different operators, different types of content delivery increase the complexity of the network, as well as the CapEx and OpEx [17]. Moreover, content delivery is a very volatile market with new protocols, content formats, device types, etc. With dedicated physical resources, operators do not have the flexibility to react on these rapid changes. Fortunately, WNV enables the sharing of not only the infrastructure, but also the content, among different service providers. Consequently, the CapEx and OpEx of RANs, CNs as well as content delivery, can be reduced significantly.

On the other hand, virtual resource allocation is a significant challenge of WNV. Virtual resource allocation schemes need to decide how to embed a virtual wireless network on physical networks (e.g., nodes, links and resources that should be picked and optimized). As content retrieval (instead of other traditional parameters, such as spectrum efficiency) is put as a high priority in ICN, the processes in wireless network virtualization (e.g., virtual resource abstracting, slicing, sharing and control) will be significantly affected by ICN. Therefore, jointly considering wireless network virtualization and ICN can improves the end-to-end network performance.

We propose a framework of information-centric wireless network virtualization, as shown in Fig. 2.1. In this example, based on the requests of services 1 and 2, two virtual networks are created and embedded on the substrate physical wireless networks. In the virtual network on the left hand side, ICN functions are integrated 


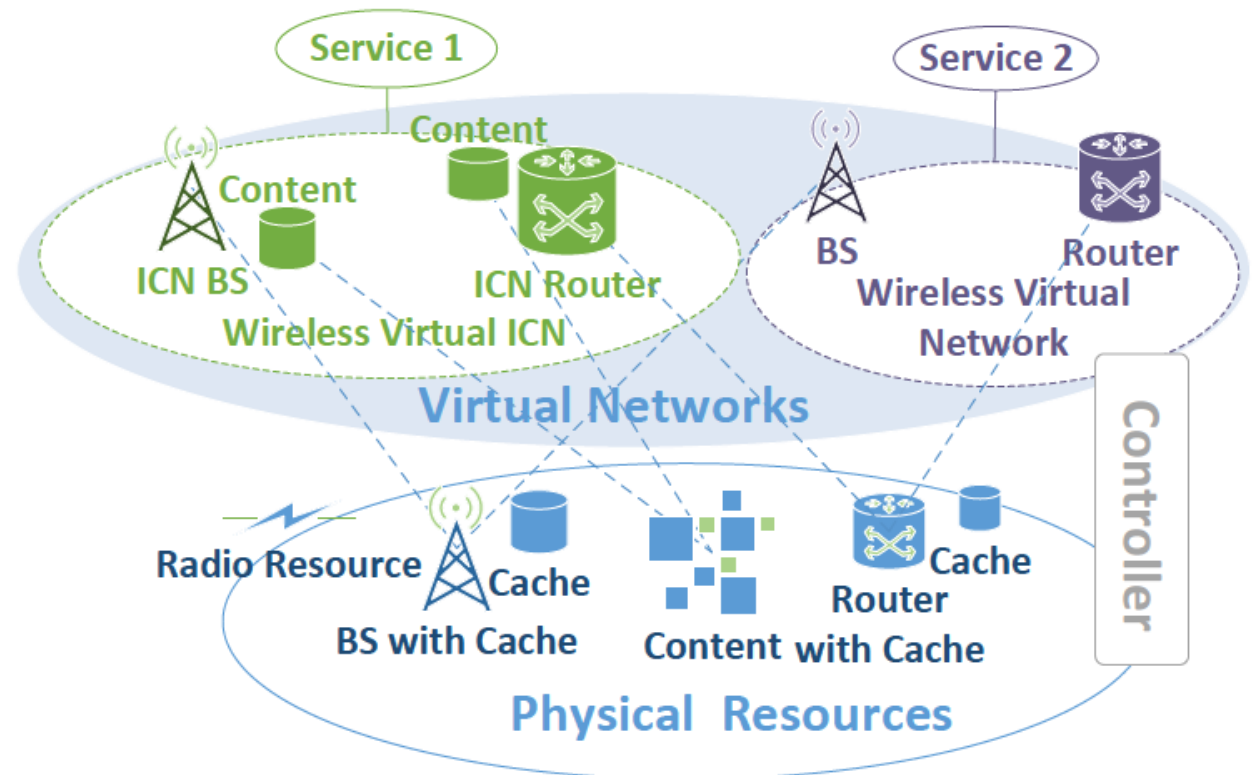

Figure 2.1: An example framework of information-centric wireless network virtualization.

and cached content can be shared. The quality-of-service (QoS) requirements and properties of content are different for different services. A personal real-time service (e.g., call, text, video meeting, etc.) prefers to request a virtual network without ICN because the contents in these services are private and with very low reuse probability. In contrast, a virtual network with ICN functions is more attractive to a streaming video service (e.g., Youtube, Netflex, etc.). Virtual ICN can provide less delay and efficient backhaul access. End users logically connect to the virtual network from where they subscribe to the service, while they physically connect to the physical network. A virtual wireless network controller needs to be deployed at the network to realize the virtualization process [17].

In this subsection, we describe a novel virtualization scheme in the proposed information-centric wireless network virtualization framework, based on the observation that MVNOs will play a key role in future mobile network markets [20]. As mentioned in the above subsections, MVNOs will lease substrate mobile networks 
from InPs and manage these networks through their own policies. After getting substrate networks, MVNOs have to virtualize and slice them, then provide virtual networks to SPs. Two important properties of virtual network are isolation and customization (or programmable) [4]. Therefore, inspired by the virtual time tagging scheme [23] and virtual resource block and bearer [75], in this chapter, we propose a flexible and simple virtualization scheme by introducing a new logical element called virtual bearer $(\mathrm{VB})$ in the proposed framework.

Specifically, each VB is a bandwidth-time resource unit. VB looks very similar to the physical resource block (PRB) in LTE systems. They are both the isolated resource units. However, there are some differences between them. Firstly, the timescale is different. The time length of each PRB is a time slot (e.g., 0.5ms in LTE), but SPs and MVNO can negotiate and pre-define the time length of VBs (might be range from seconds to days). For instance, a video SP may ask several minutes as the time length of the VB. Secondly, the owners of VBs are SPs, who may be not familiar with physical wireless networks, are just interested in QoS provided to end users. Thus, the bandwidth of VBs is data rate instead of spectrum. Moreover, the latency requirement can also be one of attributes of the VB. A well-defined VB between SPs and MVNOs is very important. By contrast, PRBs are owned and controlled by InPs. Generally speaking, VB in virtualization is a resource unit related to network services.

After defining VBs, the virtualization process is summarized in Algorithm 2.1. The VBs are generated by MVNOs based on the current status of network and content cache. At each scheduling period, SPs can allocate VBs to their own end users by their requirements of services. Let $\bar{r}$ be the data rate of each $\mathrm{VB}, \bar{t}$ be the length of these VBs, and $n_{k}$ be the number of VBs allocated to user $k$. An MVNO has to allocate proper and enough physical resources to user $k$, so that the data of user $k$ can be 
Table 2.1: Virtualization scheme in the proposed framework

Virtualizing Radio Resource and Cache in an information-centric wireless network

1: Initialization

2: Slicing: The MVNO generates certain number of virtual bearers for SPs based on the network capacity and the current network status.

3: Define virtual resources: The MVNO defines the properties (e.g., time length, data rate, and priority) for each virtual bearer based on the pre-agreements with SPs.

4: Provide virtual resources: The MVNO delivers virtual bearers to the corresponding SPs.

5: Scheduling

6: Programming: Each SP allocates appropriate number of virtual bearers to each end user based on its QoS (e.g., delay and data rate).

7: MVNO receives the scheduling information about the next potential served users from SPs.

8: Isolating: The MVNO converts the properties of each virtual bearer to data rate requirements and prepares the physical resources for each users.

9: Caching: Based on the content of users, MVNO should jointly decide the caching strategy.

10: Mapping The MVNO allocates physical resources (e.g., BS, radio resource, and cache space) to each end user based on the current network status and cache status.

transmitted by at least $\bar{r} n_{k}$ in the coming $t$ seconds. This mapping process includes not only spectrum, time slot and power, but also BSs selection (user association) and content cache, which will be discussed in the next subsection. Since the wireless channel is varying with time, it should be noted that $\bar{r} n_{k}$ is the minimum requirement of data rate, thus the actual data rate may be higher than $\bar{r} n_{k}$ to meet the requirement.

With our proposed virtualization scheme, the two important performance metrics, delay and data rate, can both be controlled by SPs. SPs can allocate resources based on their own transmission policies and even networking protocols, so that customization is realized. By using the correct mapping, isolation is also guaranteed, because 
VBs are independent with each other. An SP does not need to know the existence of other SPs, and its behavior of transmission management will not affect other SPs. Compared to existing mechanisms (e.g., defining multiple QoS levels in LTE), our proposed virtualization scheme is more flexible for SPs.

To summarize, the two important components in our proposed information-centric wireless network virtualization architecture are the virtual resource allocation scheme and the in-network caching strategy. The virtualization procedure performed by MVNOs can be considered as the mapping process between virtual resources and physical resources. Thus, MVNOs need to allocate appropriate physical resources so that the requirements of virtual resources are satisfied. The in-network caching strategy decides whether or not the content should be cached at BSs based on potential benefits (e.g., release of redundant traffic and decrease of delay). The problem is how to design an efficient scheme to consider these metrics together in the framework of information-centric wireless network virtualization.

Therefore, a feasible and efficient algorithm to enable the mapping process is the key of successfully implementing virtualization in the proposed framework. MVNOs can benefit from this algorithm, not only because it provides the solution of virtualization, but also because it can maximize MVNOs' revenue through allocating resources. We will formulate the virtual resource allocation and in-network caching strategy as an optimization problem in the following.

\subsection{System Model and Problem Statement}

In this section, we present the system model for the mobile network, virtualization, and caching. The problem is formulated after the related assumptions are given. In this chapter, we use bold capital letter to indicate matrices and vectors. $|\mathcal{S}|$ is used to indicate the number of elements of the set $\mathcal{S}$. $\lfloor a\rfloor$ means taking the maximum 
integer value that is less than $a .[x]^{+}=\max \{0, x\}$ denotes the projection function to the non-negative orthant. Other notations are summarized in Table 2.2.

\subsubsection{Network Model}

We consider a mobile cellular network with $J$ BSs. The set of BSs is $\mathcal{J}$, and $j$ is used to indicate one of the BSs. In this chapter, the cellular network is limited in an area, where there are only one macro BS and several small cell BSs. These BSs belong to different InPs with different leasing prices. Even we only consider a single macrocell system in this chapter, it can be easily extended to the multi-cell case. Let $\mathcal{K}$ denote the set of SPs. For each SP $k$, each allocated user is denoted by $i_{k}$, and $\mathcal{I}_{k}$ is the set of users of SP $k$.

The licensed spectrum used by different InPs is orthogonal, which means there is no interference between InPs. The spectrum used within in one $\mathrm{InP}$ is overlaid, which means downlink interference between macrocell and small cells as well as among small cells are both considered. The spectrum bandwidth allocated to BS $j$ is $B_{j} \mathrm{~Hz}$, and the backhaul capacity of BS $j$ is $C_{j}$ bps. Fixed equal power allocation mechanism is used, where the normalized transmit power on BS $j$ is $p_{j}$ Watts/Hz. Therefore, by using the Shannon bound, the spectrum efficiency of user $i_{k}$ who associates with BS $j$ is

$$
r_{i_{k} j}=\log _{2}\left(1+\gamma_{i_{k} j}\right)
$$

where

$$
\gamma_{i_{k} j}=\frac{g_{i_{k} j} p_{j}}{\sum_{l, l \neq j} g_{i_{k} l} p_{l}+\sigma}
$$

is the signal to interference-plus-noise ratio (SINR) between user $i_{k}$ and BS $j . g_{i_{k} j}$ 
Table 2.2: Notations of Chapter 2.

\begin{tabular}{|c|c|}
\hline Notation & Definition \\
\hline $\mathcal{J}$ & the set of mobile BS nodes (e.g., MBSs, SBSs) \\
\hline $\mathcal{K}$ & the set of SPs \\
\hline $\mathcal{I}$ & the set of UEs \\
\hline$B_{j}(\mathrm{~Hz})$ & the total spectrum bandwidth of BS $j$ \\
\hline$C_{j}$ & the backhaul capacity of BS $j$ \\
\hline$p_{j}(\mathrm{Watt} / \mathrm{Hz})$ & the normalized transmission power of BS $j$ \\
\hline$r_{i_{k} j}$ & received spectrum efficiency of user $i_{k}$ and BS $j$ \\
\hline$\gamma_{i_{k} j}$ & SINR of user $i_{k}$ and BS $j$ \\
\hline$g_{i_{k} j}$ & large scale channel gain between user $i_{k}$ and BS $j$ \\
\hline$x_{i_{k} j} \in\{0,1\}$ & the association indicator \\
\hline$\sigma(\mathrm{Watt} / \mathrm{Hz})$ & noise power spectrum density \\
\hline$y_{i_{k} j} \in[0,1]$ & the percentage of radio resource allocated by BS $j$ to user $i_{k}$ \\
\hline$R_{i_{k}}$ & the expected instantaneous data rate of user $i_{k}$ \\
\hline$\pi_{j}^{r}, \pi_{j}^{b}$ & the unit price of spectrum and backhaul of BS $j$ \\
\hline $\bar{R}_{i_{k}}$ & the requirements of data rate \\
\hline$\phi_{k}$ & the access fee of VN requested by SP $k$, charged by the MVNO \\
\hline$z_{i j} \in\{0,1\}$ & the caching strategy \\
\hline$q_{i_{k}}$ & the request rate of the content requested by user $i_{k}$ \\
\hline$o_{i j}$ & the gain getting through caching the content \\
\hline$\Delta C_{j}(\mathrm{bps})$ & the expected released bandwidth of backhaul in the future \\
\hline $\bar{R}_{j}^{b h}$ & the average single user data rate of BS $j$ \\
\hline$\Phi_{i_{k} j}^{a}, \Phi_{i_{k} j}^{c}$ & the net revenue of allocating radio resource and the saved cost \\
\hline$\chi_{j}$ & the feasible local variable set of BS $j$ \\
\hline$\lambda_{i l}^{j}$ & the Lagrange multipliers \\
\hline$[t]$ & the iteration step \\
\hline$\rho$ & the predefined augmented Lagrangian parameter \\
\hline
\end{tabular}


is the large-scale channel gain that includes path-loss and shadowing. $\sigma$ is the power spectrum density of additive white Gaussian noise.

Let $x_{i_{k} j}$ denote the association indicator, where $x_{i_{k} j}=1$ means that user $i_{k}$ associates to BS $j$; otherwise $x_{i_{k} j}=0$. Practically, each user only associates to only one BS; thus $\sum_{j \in \mathcal{J}} x_{i_{k} j}=1 . y_{i_{k} j} \in[0,1]$ is used to denote the percentage of radio resource allocated by $\mathrm{BS} j$ to user $i_{k}$, obviously

$$
\sum_{k \in \mathcal{K}, i_{k} \in \mathcal{I}_{k}} y_{i_{k} j} \leq 1
$$

The expected instantaneous data rate of user $i_{k}$ is

$$
R_{i_{k}}=\sum_{j \in \mathcal{J}} x_{i_{k} j} y_{i_{k} j} B_{j} r_{i_{k} j}
$$

In our model, we assume the backhaul bandwidth usage of user $i_{k}$ is the same as instantaneous data rate $R_{i_{k} j}$ [76]. Thus, the total required bandwidth of BS $j$ is $\sum_{k \in \mathcal{K}, i_{k} \in \mathcal{I}_{k}} R_{i_{k} j}$. Since the capacity of backhaul is limited, the following constraint of every BS mush hold,

$$
\sum_{k \in \mathcal{K}, i_{k} \in \mathcal{I}_{k}} x_{i_{k} j} y_{i_{k} j} B_{j} r_{i_{k} j} \leq C_{j}
$$

In our business model, the task of MVNOs is to lease physical resources from InPs and slice (virtualize) them to virtual network provided to SPs. Specifically, MVNO should dynamically pay the usage of radio resource (e.g., spectrum) to InPs of RANs and bandwidth (e.g., data rate) consumption to InPs of backhaul. To lease physical resources to MVNOs, InPs charge fees based on MVNOs' usage. without losing generality, we assume that the InPs of RANs and backhaul are different. The unit price of spectrum of BS $j$ is defined as $\pi_{j}^{r}$ per $\mathrm{Hz}$ and the price of backhaul is 
defined as $\pi_{j}^{b}$ per bps. We set different prices for BSs to reflect the various CAPEX and OPEX on BSs.

With virtualization where slices are bandwidth-based, each SP can schedule the next serving users and allocate necessary bandwidth to users based on their own QoS requirements. Assuming the pre-agreed bandwidth of slice allocated to SP $k$ is $\bar{R}_{k}^{s p}$, SP can allocate any data rate $\bar{R}_{i_{k}}$ to its serving user $i_{k}$ under the constraint $\sum_{i_{k} \in \mathcal{I}_{k}} \bar{R}_{i_{k}} \leq \bar{R}_{k}^{s p}$, where $\mathcal{I}_{k}$ is the set of the scheduled users of SP $i$. Thus, when the MVNO conducts the allocation of substrate resources to user $i_{k}, \bar{r}_{i_{k}}$ requested by SP $k$ should be guaranteed; otherwise the SP will not pay for this user since the agreement is not satisfied. Similar to InPs, multiple SPs are considered in our model. The access fee of VN requested by SP $k$, charged by the MVNO, is defined as $\phi_{k}$ per bps.

The caching strategy can be controlled by a binary parameter $z \in\{0,1\}[77]$. If BS $j$ caches the content that requested by user $i_{k}, z_{i_{k} j}=1$; otherwise $z_{i_{k} j}=0$. For the sake of releasing notation, we use the first user's index to index the content requested by it. Therefore, the expected reward (gain) of a certain caching strategy $\left\{z_{1 j}, z_{2 j}, \ldots, z_{I j}\right\}$ proposed in [77] is shown as

$$
\text { Reward of Caching }=\sum_{k \in \mathcal{K}} q_{k} o_{i_{k} j} z_{i_{k} j}
$$

where $q_{i_{k}}$ is the request rate of the content requested by user $i_{k}$, and $o_{i_{k} j}$ is the gain getting through caching the content.

The authors in [2] and [78] claimed that the caching gain $o_{i j}$ of mobile networks can be alleviation of bandwidth and reduction of delay. In this chapter, we choose the alleviation of backhaul bandwidth as the gain (reward) of caching. Therefore, (2.6) 
can be represented as the expected released bandwidth of backhaul in the future

$$
\Delta C_{j}=\sum_{k \in \mathcal{K}, i_{k} \in \mathcal{I}_{k}} q_{i_{k}} \bar{R}_{j}^{b h} x_{i_{k} j} z_{i_{k} j}
$$

where $\bar{R}_{j}^{b h}$ is the average single user data rate of BS $j . \Delta C_{j}$ is an estimated gain of caching, which can reflect the long term benefit of introducing cache into virtualized networks. It should be noted that the storage of BS $j$ may be limited. Thus, the cached content cannot be larger than the remaining space of cache $Z_{j}$ at $\mathrm{BS} j$. Specifically, the following constraint must hold

$$
\sum_{k \in \mathcal{K}, i_{k} \in \mathcal{I}_{k}} x_{i_{k} j} z_{i_{k} j} s_{i_{k}} \leq Z_{j}
$$

where $s_{i_{k}}$ is the size of the content requested by user $i_{k}$. In this chapter, we assume the size of all the content are the same, then $s_{i_{k}}=1$.

Obviously, the performance of caching significantly depends on the request rate (relative popularity of content) [79]. From observations and statistics [79], if the size of a content is a constant, the quest rate $q(n)$ for the $n$-th most popular content follows Zipf popularity distribution, i.e., $q(n)=1 / n^{\alpha}$, where $\alpha$ is constant. Typically, $\alpha$ is 0.56 [43]. $q(n)$ is also called user request profile (URP). Thus, if the content requested by user $i_{k}$ is known, the request rate $q_{i_{k}}$ can be derived from $q(n)$. Actually, the request rate of content is an uncertain field as the modeling of request rate is still studied by many researches. Nevertheless, this is beyond the scope of this dissertation, since we focus on a caching strategy with known request rate. 


\subsubsection{Problem Formulation}

\section{Utility Function}

Let us consider the case where user $i$ is associated with BS $j$. The revenue, radio resource cost and backhaul cost are $\phi_{k} B_{j} r_{i_{k}}, \pi_{j}^{r} B_{j}$ and $\pi_{j}^{b} B_{j} r_{i_{k} j}$, respectively. Thus, the net revenue of allocating radio resource to user $i$ is defined as

$$
\Phi_{i_{k} j}^{a}=\phi_{k} B_{j} r_{i_{k} j}-\pi_{j}^{r} B_{j}-\pi_{j}^{b} B_{j} r_{i_{k} j}
$$

According to (2.8), the expected saved backhaul by caching the content of user $i$ is $q_{i_{k}} \bar{R}_{j}$. Thus, the saved cost can be defined as

$$
\Phi_{i_{k} j}^{c}=\pi_{j}^{b} q_{i_{k}} \bar{R}_{j}
$$

Since the proportion of radio resource allocated to user $i$ is controlled by $y_{i_{k} j}$ and the caching strategy is $z_{i_{k} j}$, we can formulate the total utility of the MVNO by

$$
U_{\text {total }}=\sum_{\mathcal{I}, \mathcal{K}, \mathcal{J}} u\left(\Phi_{i_{k} j}^{a} y_{i_{k} j} x_{i_{k} j}+\Phi_{i_{k} j}^{c} z_{i_{k} j} x_{i_{k} j}\right)
$$

where $u(\cdot)$ is a utility function that is a nondecreasing and convex function normally. In this chapter, we adopt the well-known logarithmic function to our utility function. The logarithmic function has been widely used in the literature [80]. Namely,

$$
u(x)= \begin{cases}\log x & x>0 \\ -\infty & \text { otherwise }\end{cases}
$$




\section{Formulation}

In this subsection, we will formulate an optimization problem to maximize the aggregate utility of the MVNO. We provide a short overview of this subsection in the following list:

- Formulation: an optimization problem is introduced, which involves finding the association indicator $\left\{x_{i_{k} j}\right\}$, resources allocation parameters $\left\{y_{i_{k} j}\right\}$ and $\left\{z_{i_{k} j}\right\}$ corresponding to caching strategy.

- Relaxation: the binary condition of $\left\{x_{i_{k} j}\right\}$ and $\left\{z_{i_{k} j}\right\}$ are relaxed.

- Convexity: the convexity of the relaxed problem is shown by introducing an equivalent problem.

The aggregate utility maximization problem is shown as follows:

$$
\begin{aligned}
& \max _{\substack{x_{i_{k} j}, z_{i_{k} j} j\{0,1\} \\
y_{i_{k} j} \in \mathcal{R}+}} \sum_{\mathcal{I}, \mathcal{K}, \mathcal{J}} x_{i_{k} j} u\left(\Phi_{i_{k} j}^{a} y_{i_{k} j}+\Phi_{i_{k} j}^{c} z_{i_{k} j}\right) \\
& \text { s.t. } \quad C 1: \quad \quad \sum_{\mathcal{J}} x_{i_{k} j}=1, \quad \forall i, k \\
& C 2: \quad \sum_{\mathcal{I}, \mathcal{K}} x_{i_{k} j} y_{i_{k} j} \leq 1, \quad \forall j \\
& C 3: \quad \sum_{\mathcal{I}, \mathcal{K}} x_{i_{k} j} y_{i_{k} j} B_{j} r_{i_{k} j} \leq C_{j}, \quad \forall j \\
& C 4: \quad \sum_{\mathcal{J}} x_{i_{k} j} y_{i_{k} j} B_{j} r_{i_{k} j} \geq \bar{R}_{i_{k}}, \quad \forall i, k \\
& C 5: \quad \sum_{\mathcal{I}, \mathcal{K}} x_{i_{k} j} z_{i_{k} j} \leq Z_{j}, \quad \forall j .
\end{aligned}
$$

It is equivalent to take $x_{i_{k} j}$ outside the utility function without losing any optimality. If $x=1$, we have $x u(y, z)=u(x, y, z)$; if $x=0$ that means user is not served by BS so that no resource will be allocated, $u(x, y, z)=0$ and $x u(y, z)=0 . x_{i_{k} j}$ and $z_{i_{k} j}$ are Boolean. The first constraint in problem (2.13) enforces that users can only 
be associated with one BS at the same time. Constraints $\mathrm{C} 2$ and $\mathrm{C} 3$ reflect that the sum of allocated resource of all users being served by BS $j$ cannot exceed the total radio resource and backhaul bandwidth. Inequality (2.13) $\mathrm{C} 4$ is due to the minimum virtualization data rate requirements. The constraints of (2.13) C5 ensure that the caching strategy is limited in the empty space of the cache of each BS. $x_{i_{k} j} y_{i_{k} j}$ and $x_{i_{k} j} z_{i_{k} j}$ appeared in $\mathrm{C} 2$ to $\mathrm{C} 5$ are equivalent to $y_{i_{k} j}$ and $z_{i_{k} j}$. Explanation is in Proposition 2.3.1. We use $x_{i_{k} j} y_{i_{k} j}$ instead of $y_{i_{k} j}$, because it is more convenient to transfer it to a convex problem.

Problem (2.13) is difficult to solve based on the following observations:

- The feasible set of (2.13) is non-convex as a result of the binary variables $\left\{x_{i_{k} j}\right\}$ and $\left\{z_{i_{k} j}\right\}$.

- The objective function is not convex due to the product relationship between $\left\{x_{i_{k} j}\right\}$ and convex function of $\left\{y_{i_{k} j}\right\}$ as well as $\left\{z_{i_{k} j}\right\}$.

- The size of the problem is very large. The number of variables in this problem can reach more than $I^{J}$. In future cellular networks, the density and number of small cells will rise significantly so that the number of variables $\left\{x_{i_{k} j}\right\},\left\{y_{i_{k} j}\right\}$ as well as $\left\{z_{i_{k} j}\right\}$ will become very large. In addition, even only considering the user association problem, a central algorithm has the complexity of $O\left(I^{J}\right)$ to find a global optimum, which makes it unsuitable for practical networks. Moreover, in this Chapter, only small cells are considered, while in future networks, relays, and pico cells need to be considered as well, which result in even larger size.

As is well known, a mixed discrete and non-convex optimization problem is expected to be NP-hard [81,82], and it is very challenging to find its global optimum. Thus, we have to simplify problem (2.13). A relaxation of the binary conditions of $\left\{x_{i_{k} j}\right\}$ and $\left\{z_{i_{k} j}\right\}$ constitutes the first step of solving the problem. 


\subsubsection{Problem Transformation}

Following the approach in [81] and [82], we relax $x_{i_{k} j}$ and $z_{i_{k} j}$ in the problem (2.13) to be real value variables, $0 \leq x_{i_{k} j} \leq 1$ and $0 \leq z_{i_{k} j} \leq 1$. The relaxed $x_{i_{k} j}$ can be sensible and meaningfully interpreted as the time sharing factor that represents the ratio of time when user $i_{k}$ associates with BS $j$ [82]. Similar to $x_{i_{k}}$, the relaxed $z_{i_{k} j}$ can be interpreted as the time fraction of sharing one unit cache.

However, even after relaxing the variables, the problem is still non-convex due to the non-convex objective function. Thus, to make problem (2.13) tractable and solvable, a second step is necessary. Firstly, we give a proposition of the equivalent problem of (2.13).

Proposition 2.3.1 If we define $\tilde{y}_{i_{k} j}=y_{i_{k} j} x_{i_{k} j}, \quad \tilde{z}_{i_{k} j}=z_{i_{k} j} x_{i_{k} j}$ and $x_{i_{k} j} u\left[\left(a_{i_{k} j} \tilde{i}_{i_{k} j}+b_{i_{k} j} \tilde{z}_{i_{k} j}\right) / x_{i_{k} j}\right]=0$ for $x_{i_{k} j}=0$, there exists an equivalent formulation of problem (2.13) as follows:

$$
\begin{array}{crr}
\max & \sum_{x_{i_{k} j}, \tilde{y}_{i_{k} j}, \tilde{z}_{i_{k} j} \in[0,1]} x_{i_{k} j} u\left(\frac{\Phi_{i_{k} j}^{a} \tilde{y}_{i_{k} j}+\Phi_{i_{k} j}^{c} \tilde{z}_{i_{k} j}}{x_{i_{k} j}}\right) \\
\text { s.t. } & C 1: & \sum_{\mathcal{J}} x_{i_{k} j}=1, \quad \forall i, k \\
& \tilde{C} 2: & \sum_{\mathcal{I}, \mathcal{K}} \tilde{y}_{i_{k} j} \leq 1, \quad \forall j \\
\tilde{C} 3: & \sum_{\mathcal{I}, \mathcal{K}} \tilde{y}_{i_{k} j} B_{j} r_{i_{k} j}-C_{j} \leq 0, \quad \forall j \\
\tilde{C} 4: & \bar{R}_{i_{k}}-\sum_{\mathcal{J}} \tilde{y}_{i_{k} j} B_{j} r_{i_{k} j} \leq 0, \quad \forall i, k \\
\tilde{C} 5: & \sum_{\mathcal{I}, \mathcal{K}} \tilde{z}_{i_{k} j}-Z_{j} \leq 0, \quad \forall j .
\end{array}
$$

Proof This proof of Proposition 2.3.1 is motivated by [83]. The relaxed problem (2.13) can be recovered by substitution of variable $\tilde{y}_{i_{k} j}=y_{i_{k} j} x_{i_{k} j}$ and $\tilde{z}_{i_{k} j}=z_{i_{k} j} x_{i_{k} j}$ into problem (2.14) except $x_{i_{k} j}=0$. Due to the loss of definition when $x_{i_{k} j}=0$, it 
is not a one-to-one mapping. However, if $x_{i_{k} j}=0, y_{i_{k} j}=0$ certainly holds because of the optimality. Obviously, BS $j$ does not allocate any resources to any user if the user does not associate with BS $j$. Thus, it becomes a one-to-one mapping when the completed mapping between $\left\{x_{i_{k} j}, y_{i_{k} j}, z_{i_{k} j}\right\}$ and $\left\{x_{i_{k} j}, \tilde{y}_{i_{k} j}, \tilde{z}_{i_{k} j}\right\}$ is

$$
y_{i_{k} j}= \begin{cases}\tilde{y}_{i_{k} j} / x_{i_{k} j} & x_{i_{k} j}>0 \\ 0 & \text { otherwise }\end{cases}
$$

and

$$
z_{i_{k} j}= \begin{cases}\tilde{z}_{i_{k} j} / x_{i_{k} j} & x_{i_{k} j}>0 \\ 0 & \text { otherwise. }\end{cases}
$$

With Proposition 2.3.1 and the well-known perspective function [84], we can have the following theory that gives the convexity of problem (2.14).

\subsubsection{Convexity}

Theorem 2.3.2 If problem (2.14) is feasible, it is jointly convex with respect to all optimization variables $x_{i_{k} j}, \tilde{y}_{i_{k} j}, \tilde{z}_{i_{k} j}, \forall i, j, k$.

Proof The proof of the convexity is similar to [83], we describe briefly as follows. Firstly, we prove the continuity of the function $f(t, x)=x \log (t / x), t \geq 0, x \geq 0$ at the point $x=0$. Let $s=t / x$,

$$
f(t, 0)=\lim _{x \rightarrow 0} x \cdot \log \frac{t}{x}=\lim _{s \rightarrow \infty} \frac{t}{s} \log s=t \lim _{s \rightarrow \infty} \frac{\log s}{s}=0 .
$$

$f(t, x)=x \log (t / x), t \geq 0, x \geq 0$ is the well-known perspective operation [84] of $\log$, 
where convexity is preserved. The perspective function of a concave function is a concave function.

Since $x_{i_{k} j} \log \left[\left(a_{i_{k} j} \tilde{y}_{i_{k} j}+b_{i_{k} j} \tilde{z}_{i_{k} j}\right) / x_{i_{k} j}\right]=0$ for $x_{i_{k} j}=0$ and $\left(a_{i_{k} j} \tilde{y}_{i_{k} j}+b_{i_{k} j} \tilde{z}_{i_{k} j}\right)$ is nothing but a linear combination of $y$ and $z, x_{i_{k} j} \log \left[\left(a_{i_{k} j} \tilde{y}_{i_{k} j}+b_{i_{k} j} \tilde{z}_{i_{k} j}\right) / x_{i_{k} j}\right]$ is the perspective function of $\log \left(a_{i_{k} j} \tilde{y}_{i_{k} j}+b_{i_{k} j} \tilde{z}_{i_{k} j}\right)$ that is concave. Our objective function in problem (2.14) is a sum of concave functions, and all of the constraints are linear. Thus, problem (2.14) is a convex problem.

Since problem (2.14) is a convex problem, a lot of methods (e.g., interior point method) can be used to solve it. However, as we mentioned above, with the increase in the number of BSs, the size of problem will be very large. Practically, even with a powerful computing center, the overhead of delivering enough local information (e.g., channel status information [CSI]) to a global center is extremely inefficient. Therefore, for the purpose of implementation, a distributed algorithm running on each BS should be adopted. But, due to the constrains $C 1, \tilde{C} 3-\tilde{C} 5$ of problem (2.14), the problem is not separable with respect to the BSs. Specifically, the user association indicator that represents the connection between users and BSs is a global variable. Thus, in order to achieve a decentralized optimization algorithm, this coupling has to be decoupled appropriately, which is discussed in the following.

\section{Decoupling of Association Indicators}

In order to solve problem (2.14), we introduce local copies of the global association indicators. Each local variable can be considered as the preference of each BS about the association of users. Let us introduce a set of new variables to represent the local copies of our association indicators. To lighten the notation, from now on, we use $i$ to denote all the users instead of $i_{k}$. If we define $\mathrm{x}$ as the vector of association indicators 
$\left\{x_{i l}, \forall l, k\right\}^{1}$, the local copy of $\mathbf{x}$ at BS $j$ is denoted as $\hat{\mathbf{x}}^{j}$. Formally,

$$
\hat{x}_{i l}^{j}=x_{i l}, \forall j, k, l .
$$

By means of the local vectors $\hat{\mathbf{x}}^{j}, \tilde{\mathbf{y}}_{j}$ and $\tilde{\mathbf{z}}_{j}$, let us define a feasible local variable set for each BS $j \in \mathcal{J}$,

$$
\chi_{j}=\left\{\begin{array}{l|l}
\hat{\mathbf{x}}^{j}, \tilde{\mathbf{y}}_{j}, \tilde{\mathbf{z}}_{j} & \begin{array}{l}
\hat{x}_{i l}^{j}, \tilde{y}_{i_{k} j}, \tilde{z}_{i_{k} j} \in[0,1], \forall i, j, l \\
\sum_{\mathcal{J}} \hat{x}_{i l}^{j}=1, \forall j, k \\
\sum_{\mathcal{K}} \tilde{y}_{i j} \leq 1, \forall j \\
\sum_{\mathcal{K}} \tilde{y}_{i j} B_{j} r_{i j} \leq C_{j}, \forall j \\
\sum_{\mathcal{J}} \tilde{y}_{i j} B_{j} r_{i j} \geq r_{k}, \forall k \\
\sum_{\mathcal{K}} \tilde{z}_{i j} \leq Z_{j}, \forall j
\end{array}
\end{array}\right\} .
$$

and an associated local utility function, respectively, as

$$
g_{j}= \begin{cases}-\sum_{\mathcal{K}} \hat{x}_{i l}^{j} u\left[\frac{\left(\Phi_{i j}^{a} \tilde{i}_{i j}+\Phi_{i j}^{c} \tilde{z}_{i j}\right)}{\hat{x}_{i l}^{j}}\right] & \hat{\mathbf{x}}^{j}, \tilde{\mathbf{y}}_{j}, \tilde{\mathbf{z}}_{j} \in \chi_{j} \\ \infty & \text { otherwise }\end{cases}
$$

With this notation, the global consensus problem of problem (2.14) can be written

\footnotetext{
${ }^{1}$ Note that we need to differentiate the real BS from the copy of other BSs. Therefore, we keep the index of BS as $j$, and we introduce another index, $l$, to denote the copied information of other BSs in BS $j$.
} 
as

$$
\begin{aligned}
& \min \quad G\left(\hat{\mathbf{x}}^{j}, \tilde{\mathbf{y}}_{j}, \tilde{\mathbf{z}}_{j}\right)=\sum_{\mathcal{J}} g_{j}\left(\hat{\mathbf{x}}^{j}, \tilde{\mathbf{y}}_{j}, \tilde{\mathbf{z}}_{j}\right) \\
& \text { s.t. } \quad \hat{x}_{i l}^{j}=x_{i l}, \forall i, j, l .
\end{aligned}
$$

Obviously, our objective function is separable across the BSs in the network. However, the global association variables are still involved in the consensus constraints. In the next section, we will apply ADMM to solve problem (2.19) in a distributed manner.

\subsection{Virtual Resource Allocation and In-Network Caching via ADMM}

In this section, we apply ADMM to solve problem (2.19). The proposed algorithm for virtual resource allocation and in-network caching via ADMM is described. A brief description of ADMM is given in Appendix A.

According to [74], our problem (2.19) is a global consensus problem, since the constraint is that all the local variables should agree. The initial step to apply ADMM to problem (2.19) is that an augmented Lagrangian with corresponding global consensus constrains should be formed. Let $\lambda_{i l}^{j}, \forall j \in \mathcal{J}, l \in \mathcal{J}, i \in \mathcal{I}$ be the Lagrange multipliers corresponding to the consensus constraints in problem (2.19). The augmented 
Lagrangian for problem (2.19) is

$$
\begin{aligned}
& \mathfrak{L}_{\rho}\left(\left\{\hat{\mathbf{x}}^{j}, \tilde{\mathbf{y}}_{j}, \tilde{\mathbf{z}}_{j}\right\}_{j \in \mathcal{J}},\{\mathbf{x}\},\left\{\lambda^{j}\right\}\right)=\sum_{j \in \mathcal{J}} g_{j}\left(\hat{\mathbf{x}}^{j}, \tilde{\mathbf{y}}_{j}, \tilde{\mathbf{z}}_{j}\right) \\
& +\sum_{\substack{i \in \mathcal{I} \\
j, l \in \mathcal{J}}} \lambda_{i l}^{j}\left(\hat{x}_{i l}^{j}-x_{i l}\right)+\frac{\rho}{2} \sum_{\substack{i \in \mathcal{I} \\
j, l \in \mathcal{J}}}\left(\hat{x}_{i l}^{j}-x_{i l}\right)^{2},
\end{aligned}
$$

where $\lambda^{j}$ is the vector of the Lagrange multipliers and $\rho \in \mathbb{R}_{++}$is a positive constant parameter for adjusting the convergence speed of the ADMM [74]. Based on the iteration of ADMM with consensus constraint [74], the ADMM method applied to problem (2.19) consists of following sequential optimization steps:

$$
\begin{aligned}
& \left\{\hat{\mathbf{x}}^{j}, \tilde{\mathbf{y}}_{j}, \tilde{\mathbf{z}}_{j}\right\}_{j \in \mathcal{J}}^{[t+1]}:= \\
& \arg \min \left\{\begin{array}{l}
g_{j}\left(\hat{\mathbf{x}}^{j}, \tilde{\mathbf{y}}_{j}, \tilde{\mathbf{z}}_{j}\right) \\
+\sum_{\substack{i \in \mathcal{I} \\
l \in \mathcal{J}}} \lambda_{i l}^{j[t]}\left(\hat{x}_{i l}^{j}-x_{i l}^{[t]}\right) \\
+\frac{\rho}{2} \sum_{\substack{i \in \mathcal{I} \\
l \in \mathcal{J}}}\left(\hat{x}_{i l}^{j}-x_{i l}^{[t]}\right)^{2}
\end{array}\right\} . \\
& \{\mathbf{x}\}^{[t+1]}:= \\
& \arg \min \left\{\begin{array}{c}
\sum_{j \in \mathcal{J}} \sum_{\substack{i \in \mathcal{I} \\
l \in \mathcal{J}}} \lambda_{i l}^{j[t]}\left(\hat{x}_{i l}^{j[t+1]}-x_{i l}\right) \\
+\frac{\rho}{2} \sum_{j \in \mathcal{J}} \sum_{\substack{i \in \mathcal{I} \\
l \in \mathcal{J}}}\left(\hat{x}_{i l}^{j[t+1]}-x_{i l}\right)^{2}
\end{array}\right\} . \\
& \left\{\lambda_{j}\right\}_{j \in \mathcal{J}}^{[t+1]}:=\lambda_{j}^{[t]}+\rho\left(\hat{\mathbf{x}}^{j[t+1]}-\mathbf{x}^{[t+1]}\right) .
\end{aligned}
$$


Obviously, the first step (2.21) and third step (2.23) can be completely decentralized, which find the local optimum association, radio resource allocation and caching strategies, as well as local Lagrange multipliers. The second step (2.22) can be optimized by a center controller of MVNO. Each step will be presented in the following part of this section.

\subsection{1 $\left\{\hat{\mathbf{x}}^{j}, \tilde{\mathbf{y}}_{j}, \tilde{\mathbf{z}}_{j}\right\}_{j \in \mathcal{J} \text {-update }}$}

In step 1, local association, radio resource allocation and caching strategies are separable across each BS $j$. Therefore, $\left\{\hat{\mathbf{x}}^{j}, \tilde{\mathbf{y}}_{j}, \tilde{\mathbf{z}}_{j}\right\}_{j \in \mathcal{J}}$-update can be decomposed into $J$ subproblems, which can be solved locally at each BS. Thus, each BS $j$ solves the following optimization problem at iteration $t$ :

$$
\begin{aligned}
& \min _{\left\{\hat{\mathbf{x}}^{j}, \tilde{\mathbf{y}}_{j}, \tilde{\mathbf{z}}_{j}\right\}} \mathfrak{L}_{\rho}^{j}=g_{j}\left(\hat{\mathbf{x}}^{j}, \tilde{\mathbf{y}}_{j}, \tilde{\mathbf{z}}_{j}\right)+h_{j}\left(\hat{\mathbf{x}}^{j}\right) \\
& \text { s.t. } \quad \sum_{l \in \mathcal{J}} \hat{x}_{i l}^{j}=1, \forall i,
\end{aligned}
$$

where

$$
h_{j}\left(\hat{\mathbf{x}}^{j}\right)=\sum_{\substack{i \in \mathcal{I} \\ l \in \mathcal{J}}} \lambda_{i l}^{j}\left(\hat{x}_{i l}^{j}-x_{i l}\right)+\frac{\rho}{2} \sum_{\substack{i \in \mathcal{I} \\ l \in \mathcal{J}}}\left(\hat{x}_{i l}^{j}-x_{i l}\right)^{2} .
$$

Apparently, problem (2.24) is a convex problem but still intractable to compute the closed form solution. Hence, it should be solved by some general numerical methods (e.g., interior-point methods and successive approximation methods) that provide efficient ways. In this chapter, we use the primal-dual interior-point method [84] that provides efficient way for convex problems. The algorithm is summarized in Table 2.3 . 
Table 2.3: Algorithm for updating local variables

Primal-dual interior-point method for $\left\{\hat{\mathbf{x}}^{j}, \tilde{\mathbf{y}}_{j}, \tilde{\mathbf{z}}_{j}\right\}_{j \in \mathcal{J}}$-update

1: Initialization

2: Given $\left\{\hat{\mathbf{x}}^{j}, \tilde{\mathbf{y}}_{j}, \tilde{\mathbf{z}}_{j}\right\} \in \chi_{j}, \nu>0, \mu>1, \epsilon_{\text {feas }}>0, \epsilon>0$

3: for $\left\|r_{\text {pri }}\right\|_{2} \geq \epsilon_{\text {feas }},\left\|r_{\text {dual }}\right\|_{2} \geq \epsilon_{\text {feas }}$, and $\hat{\eta} \geq \epsilon$ do

4: $\quad$ Determine $s$. Set $t:=\mu m / \hat{\eta}$.

5: $\quad$ Compute primal-dual search direction $\Delta d_{p d}$.

6: $\quad$ Line Search and update.

7: $\quad$ Determine step length $s>0$ and set $d:=d+s \Delta d_{p d}$.

8: end for

9: Output the optimal variable $\left\{\hat{\mathbf{x}}^{j}, \tilde{\mathbf{y}}_{j}, \tilde{\mathbf{z}}_{j}\right\}$ and transmit it to the MVNO central controller.

In Table $2.3, \hat{\eta}$ is the surrogate duality gap. $m$ is the number of constraints, which is 5 in problem (2.24). $t$ used in the computing of $\Delta d_{p d}$ is set to a factor $\mu$ times $m / \hat{\eta} . \Delta d_{p d}$ is the primal-dual search direction. Detailed step-by-step description of the primal-dual interior method is given in [84], where the theory background and analysis of the primal-dual interior-point method can be also found. The only thing we should note is giving the initial feasible solution. It is easy to set the variables except $\left\{\hat{\mathbf{x}}^{j}, \tilde{\mathbf{y}}_{j}, \tilde{\mathbf{z}}_{j}\right\}$. Considering some special cases, it is not very hard to provide a feasible solution of $\left\{\hat{\mathbf{x}}^{j}, \tilde{\mathbf{y}}_{j}, \tilde{\mathbf{z}}_{j}\right\} \in \chi_{j}$. Let us consider a very simple example. Supposing that at least one user is in the coverage of BS $j$ where the QoS requested by SP can be guaranteed, BS $j$ set $\hat{x}_{i j}^{j}=1$ and remaining $\left\{\hat{x}_{i^{\prime} j}^{j}\right\}=\{0\}, i^{\prime} \neq i$, which means BS $j$ only associates with user $i$. Since only one user is associated with BS $j$, all the radio resource and cache will be allocated to this user. The last four sets of constraints in (2.18) are satisfied automatically. Moreover, in our algorithm, BS $j$ also needs to give the opinion of other BSs' association without knowing the CSI. Thus, BS $j$ randomly chooses BS $j^{\prime} \in \mathcal{J}, j^{\prime} \neq j$, for the rest of users $i^{\prime} \in \mathcal{I}, i^{\prime} \neq i$, such that each user has associated with one and only one BS. After this, the first sets of constraints 
are guaranteed.

Recall that we have relaxed the association indicator to a real value between zero and one instead of a binary variable in Subsection 2.3.3. Thus, we have to recover it to binary after we get the optimum solution of (2.21). The binary recovery deals with computing the marginal benefit for each user $k$. Then, the indicator $\{\mathrm{x}\}$ can be recovered by

$$
x_{i l}^{j}=\left\{\begin{array}{l}
1 \text { if } Q_{n l}^{j}=\max _{k}\left\{Q_{i l}^{j}, \forall k\right\} \text { and } Q_{i l}^{j}>0, \\
0 \text { otherwise }
\end{array}\right.
$$

where $Q_{i l}^{j}=\partial \mathfrak{L}_{\rho}^{j} / \partial x_{i l}^{j}$ is the first partial derivation of $x_{i l}^{j}$.

\subsection{2 $\{\mathrm{x}\}$-update and $\left\{\lambda_{j}\right\}$-update}

Compared with the updating of local variables, $\{\mathrm{x}\}$-update and $\left\{\lambda_{j}\right\}$-update are quite simple since they are only an un-constrained quadratic optimization problems. After the collection of $\left\{\hat{\mathbf{x}}^{j}, \tilde{\mathbf{y}}_{j}, \tilde{\mathbf{z}}_{j}\right\}$ and $\left\{\lambda_{j}\right\},\{\mathbf{x}\}$-update can be done by many efficient ways [84]. In this chapter, we use an interior-point method in the simulations.

\subsubsection{Stop Criteria and Convergence}

Based on the discussion in [74], our ADMM-based algorithm iterates satisfy residual convergence, objective convergence and dual variable convergence as $t \rightarrow \infty$, because our objective function $\sum_{\mathcal{J}} g_{j}\left(\hat{\mathbf{x}}^{j}, \tilde{\mathbf{y}}_{j}, \tilde{\mathbf{z}}_{j}\right)$ is closed, proper and convex and the Lagrangian $\mathfrak{L}_{\rho}$ has saddle point. The convexity of the objective function has been proven in Section 2.3.4. Obviously, all of our variables are bounded and our objective function is also bounded, so that the optimal solution $\left(\hat{\mathbf{x}}^{j \star}, \tilde{\mathbf{y}}_{j}^{\star}, \tilde{\mathbf{z}}_{j}^{\star}\right)$ of problem (2.19) satisfies $G\left(\hat{\mathbf{x}}^{j \star}, \tilde{\mathbf{y}}_{j}^{\star}, \tilde{\mathbf{z}}_{j}^{\star}\right)<\infty$. Since problem (2.19) is convex problem that means the strong 
duality holds [84], $\mathfrak{L}_{\rho}\left(\left\{\hat{\mathbf{x}}^{j}, \tilde{\mathbf{y}}_{j}, \tilde{\mathbf{z}}_{j}\right\}_{j \in \mathcal{J}}^{\star},\{\mathbf{x}\}^{\star},\left\{\lambda^{j}\right\}^{\star}\right) \leq \mathfrak{L}_{\rho}\left(\left\{\hat{\mathbf{x}}^{j}, \tilde{\mathbf{y}}_{j}, \tilde{\mathbf{z}}_{j}\right\}_{j \in \mathcal{J}},\{\mathbf{x}\},\left\{\lambda^{j}\right\}^{\star}\right)$

In [74], it is suggested that a reasonable termination criterion is that the primal residuals $s_{j}^{[t+1]}$ and dual residuals $s_{d}^{[t+1]}$ must be small,

$$
\left\|s_{j}^{[t+1]}\right\|_{2} \leq \zeta^{p r i}, \forall j
$$

and

$$
\left\|s_{d}^{[t+1]}\right\|_{2} \leq \zeta^{\text {dual }}
$$

where $\zeta^{\text {pri }}>0$ and $\zeta^{\text {dual }}>0$ are the feasibility tolerances for the primal and dual feasibility conditions, respectively. The primal residuals (that is the residual for the primal feasibility condition) of $j$ th BSs at iteration $t+1$ in this chapter can be defined as

$$
s_{j}^{[t+1]}=\hat{\mathbf{x}}^{j[t+1]}-\mathbf{x}^{[t+1]} .
$$

The dual residuals (a residual for the dual feasibility condition) at iteration $t+1$ can be defined as

$$
s_{d}^{[t+1]}=\mathrm{x}^{[t+1]}-\mathrm{x}^{[t]} .
$$

Detailed description of stop criteria of ADMM can be seen in [74].

\subsubsection{Feasibility, Complexity and Implementations}

Our proposed problem becomes infeasible if the resource allocator is unable to satisfy the QoS requirements of at least one of the virtual network users. The feasibility of problem (2.14) can be guaranteed by an admission control policy. The admission 
Table 2.4: Algorithm for virtual resource allocation

\section{Distributed virtual resource allocation via ADMM}

1: Initialization

2: At each BS $j$, collect CSI of all users within its coverage.

3: Initialize $\mathbf{x}^{0} \in \chi, \boldsymbol{\lambda}^{0}>\mathbf{0}$ and a stop criterion threshold $\zeta>0$ at the MVNO controller.

4: for $t=0,1,2, \ldots$, do

5: $\quad$ Broadcast $\mathbf{x}^{[t]}$ and $\lambda^{[t]}$ to each BSs;

6: $\quad$ At each BS $j$, solve problem (2.24) to update $\left\{\hat{\mathbf{x}}^{j}, \tilde{\mathbf{y}}_{j}, \tilde{\mathbf{z}}_{j}\right\}_{j \in \mathcal{J}}^{[t+1]}$.

7: At the controller of MVNO, update $\mathrm{x}^{[t+1]}$ by combining the results of $\left\{\hat{\mathbf{x}}^{j}, \tilde{\mathbf{y}}_{j}, \tilde{\mathbf{z}}_{j}\right\}_{j \in \mathcal{J}}^{[t+1]}$ from each BS.

8: $\quad$ Update $\mathbf{x}^{[t+1]}$ according to (2.22) and broadcast $\mathbf{x}^{[t]}$ to each BSs

9: $\quad$ Update $\boldsymbol{\lambda}^{[t]}$ via (2.23) at the controller of MVNO

10: $\quad$ if $s_{\text {dual }}^{[t+1]}=\mathrm{x}^{[t+1]}-\mathrm{x}^{[t]}$ and $\left\|s_{j}^{[t+1]}\right\|_{2} \leq \zeta^{\text {pri }}$, then go to Step 10.

11: end for

12: Output the optimal resource allocation policy $\left\{\mathrm{x}^{[t+1]}, \tilde{\mathbf{y}}_{j}, \tilde{\mathbf{z}}_{j}\right\}_{j \in \mathcal{J}}^{[t+1]}$.

control can constrain the total number of virtual networks and users. The admission control issue will be studied in the next chapter. Since the number of BSs in HetNet is comparable to the number of users [85], it is not hard to guarantee the feasibility.

Assume the total numbers of users and BSs are $I$ and $J$, respectively. At each iteration step, the computational complexity of our proposed ADMM at each BS and central controller are both $\mathcal{O}(I \times J)$. If our iteration step is $T$, the total computational complexity is $\mathcal{O}(T \times I \times J)$. Compared to the brutal central algorithm that has the computational complexity of $\mathcal{O}\left(I^{j}\right)$, our proposed algorithm can reduce the complexity significantly.

Based on the analysis above, the distributed wireless virtualization algorithm via ADMM can be summarized as Table 2.4.

According to the discussions above, the proposed ADMM algorithm can be implemented in a distributed manner across the BSs and the controller of the MVNO. The 
BSs solve their corresponding subproblems in parallel in each iteration to optimize their local variables using local CSI information and transmit their local results to the controller of the MVNO. Then, the controller collects all the local results and coordinates all the BSs to achieve the global consensus based on the consensus constraint. In this way, there is no need to exchange CSI repeatedly between the BSs and controller, which will reduce the signaling overhead significantly. Furthermore, since the combinatorial nature and non-convexity of the considered problem have been removed through the problem transformation in Subsection 2.3.3, the computational complexity to solve the problem has been reduced to a reasonable level and hence the proposed algorithm is attractive in practical networks.

Furthermore, our proposed scheme is compatible with the Network Function Virtualization (NFV) framework [17] in the European Telecommunications Standards Institute (ETSI). Firstly, in the NFV framework, the virtualization layer is the bridge between virtual functions and infrastructure, where infrastructure can be virtualized to virtual resources. Our resource allocation algorithm can be implemented at this layer to virtualize radio resources, BSs, and in-network cache to virtual resource block and virtual cache. Moreover, the virtualized infrastructure manager used to manage the virtualization layer and infrastructure can store our algorithm and adjust it according to network dynamics.

From the perspective of virtual network function (VNF), our proposed scheme can be considered as a customizable service to SPs. Furthermore, since we have considered in-network caching, this content sharing function can also be considered as a content sharing VNF, which is compatible with the NFV framework. A similar VNF called virtual content deliver network ( $\mathrm{vCDN})$ is available in the NFV framework. 


\subsection{Simulation Results and Discussions}

We use computer simulations to show the performance of the proposed scheme. The simulator is a Matlab-based system level simulator. Monto-Carlo method is used in the simulations. We run the simulations in an X86 desktop computer with quad core CPU (Intel Q8400), 4GB RAM, and the OS of Microsoft Windows 7 SP1. The position of the macro BS is fixed. We randomly distribute the small BSs and users in the covered area of the macro BS. Then, we fix the position of small BSs and change the locations of users at each shot (simulation loop). After 10,000 shots, we change the location of small BSs randomly and run 10,000 users shots again. Average values are taken to reduce the randomness effects in the simulations.

In the simulations, we consider two RAN InPs, two backhaul InPs, one MVNO, and three SPs. RAN InP 1 owns a two-tier HetNet with one macro BS with price of 100 units/MHz and 10 small BSs with price of 90 units/MHz. RAN InP 2 owns only 10 small BSs with 80 units/MHz. The price of backhaul InPs 1 for macro BS and small BSs of RAN InP 1 is 1 units/Mbps, while the price of backhaul InP 2 for small BSs of RAN InP 2 is 1.2 units/Mbps. The average numbers of the scheduled users of $3 \mathrm{SPs}$ are assumed to be equal, and the prices for requesting virtual resources are 15units/Mbps, 20 units/Mbps and 18 units/Mbps, respectively. Transmit power of $49 \mathrm{dBm}$ for macro BS and of $20 \mathrm{dBm}$ for small BSs are considered in our simulations. The bandwidth is $20 \mathrm{MHz}$. In our simulations, the location of the macro BS is fixed in the center and the locations of 20 small BSs are uniformly distributed in a area where the radius is 250 meters representing urban environment [86]. We user a path $\operatorname{loss} L(d)=34+40 \log (d)$ and $L(d)=37+40 \log (d)$ to model the macro cell and small cell propagation, respectively [81]. The lognormal shadowing with standard deviation $8 \mathrm{~dB}$ for macro cell and $4 \mathrm{~dB}$ for small cell are assumed in this chapter. The power density of thermal noise power is $-174 \mathrm{dBm} / \mathrm{Hz}$. The total content has 1000 files and 
the cache space of macro BS and small BSs is 1000 files and 100 files, respectively. The backhaul delay of small BSs is $10 \mathrm{~ms}$ longer than that of the macro BS. The backhaul capacity of the macro BS is 10Gbps and that of small BSs is 2 Gbps.

To compare our proposed scheme, three benchmarks are also considered. The first baseline is a centralized scheme based on solving problem (2.14) by interior methods directly and round up based on (2.25). The second baseline is a traditional max-SINR association scheme without virtualization [81,87], in which all users associate to the BSs who provide the maximum received SINR, and each BS performs proportional fairness resource allocation regardless of SPs. The third one is a traditional virtualization scheme mentioned as a option in [88], called hard slicing (bandwidth guarantee), in which each base station slices fixed ratio of the bandwidth to individual SPs no matter SPs use them or not.

\subsubsection{Convergence of the Proposed ADMM-based Scheme}

Fig. 2.2 shows the convergence of the proposed ADMM-based scheme and the effect of parameter $\rho$ in ADMM. The y-axis is the total utility of the MVNO, and the $\mathrm{x}$-axis is the iteration step index. We can observe from the figure that the gap between the ADMM-based scheme and the centralized scheme is narrow. This means the effectiveness of the ADMM-based scheme is equivalent to the centralized scheme considering the overall utility. It can be found that the results with different $\rho$ finally converges to almost the same utility value with only a small gap. However, $\rho$ affects the rate of convergence. $\rho=1.0$ gives higher rate than $\rho=0.5$, especially before the 100th iteration. From Fig. 2.2, we can see that there is a significant decrease of the utility gap between the centralized scheme and the proposed ADMM-based scheme from the 1st iteration to the 10th iteration. After the 10th iteration, the gain of more iterations is still increasing but with less rate. Thus, there is a tradeoff between the 


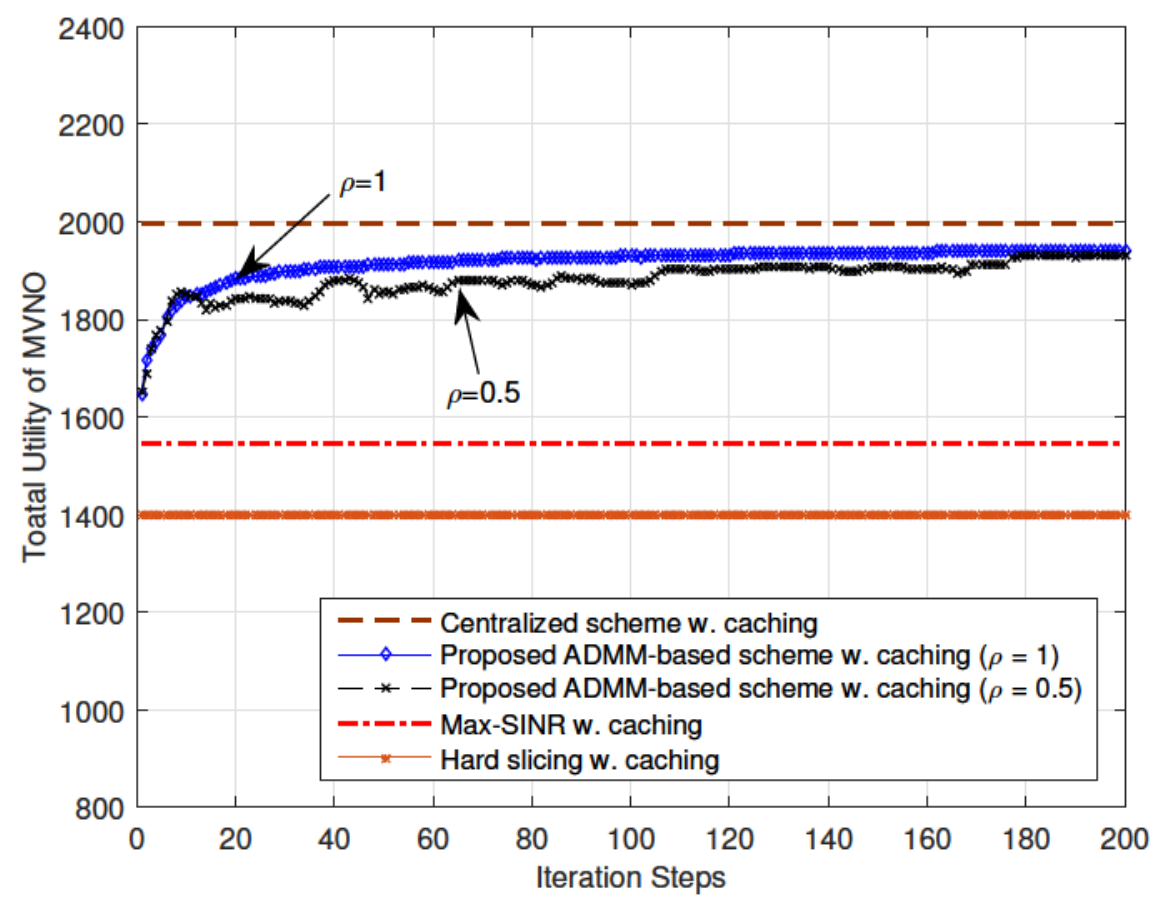

Figure 2.2: Convergence of ADMM and the effect of $\rho$.

acceptable utility value and the iteration steps.

\subsubsection{The Effects of Virtualization}

Next, the gain through wireless virtualization is evaluated in the following three aspects: 1) the incomes from SPs, 2) the maximum number of users, and 3) the required data rate of users.

Fig. 2.3a shows the average total incomes of the MVNO from all 3 SPs. The $\mathrm{x}$-axis is the number of access users and the $\mathrm{y}$-axis is the average total incomes, both from all 3 SPs. We can observe that the performance of our proposed ADMM-based scheme is better than the other two schemes. With the increase of the number of access users, the gap between them is getting wider, which means the ADMM-based scheme works better in dense environments. SPs pay more to the MVNO by using 


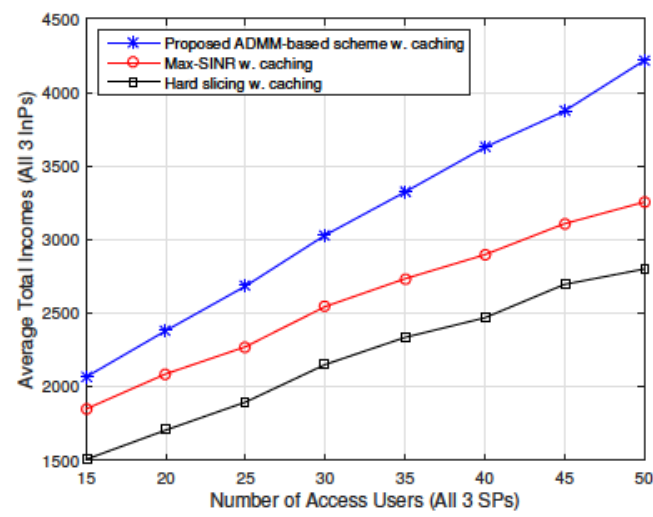

(a) All SPs

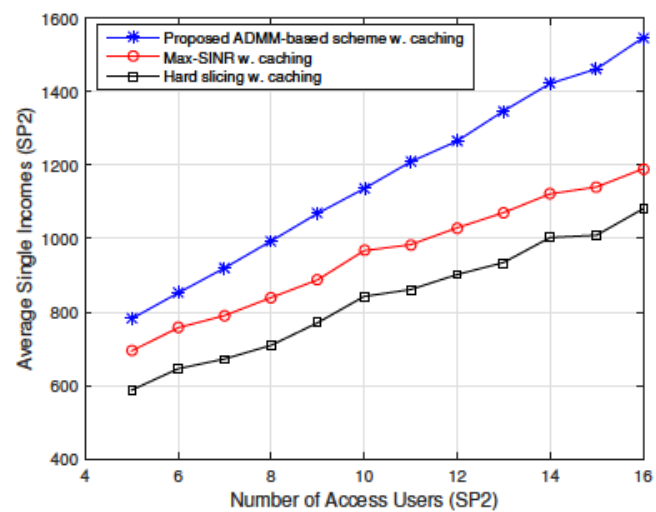

(c) SP 2

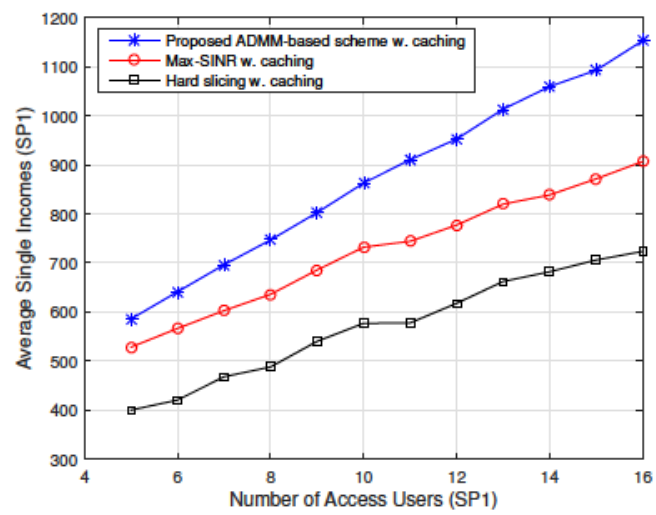

(b) SP 1

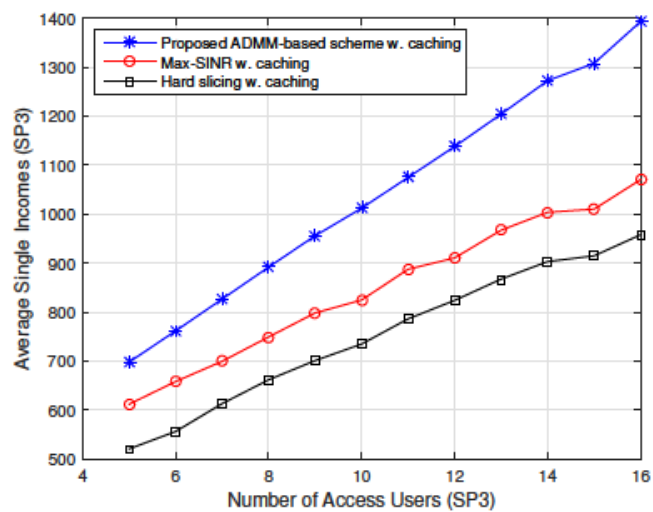

(d) SP 3

Figure 2.3: Comparison of the incomes of the MVNO.

the ADMM-based scheme, because the ADMM-based scheme can satisfy more users and achieve higher throughput than the other two schemes. Hard slicing performs the worst, because many resources are wasted. This inefficiency of hard slicing is caused by fixed resource allocation among SPs. In our simulation, we set that the unit incomes from three SPs are different (15 for SP1, 20 for SP2, and 18 for SP3). We also evaluate the income from an individual SP. As shown in Fig. 2.3b, Fig. 2.3c and Fig. 2.3d, the income from SP2 is in the range of 600 to 1600 , which is the highest due to the highest unit price of SP2.

Fig. 2.4a shows the maximum number of satisfied users, where the $\mathrm{x}$-axis is the 


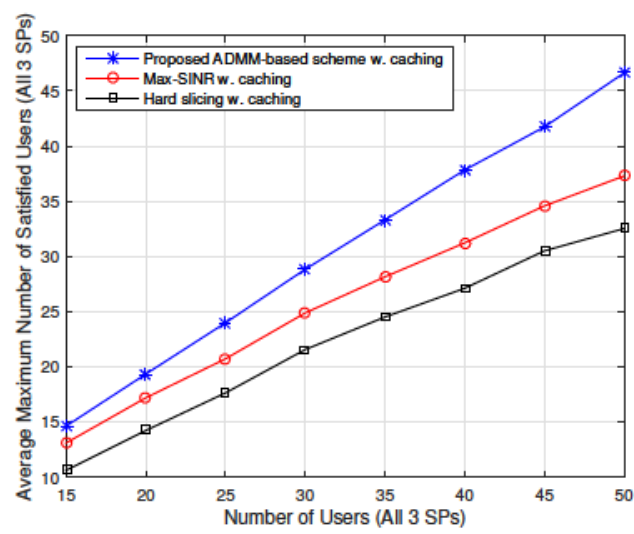

(a) All SPs

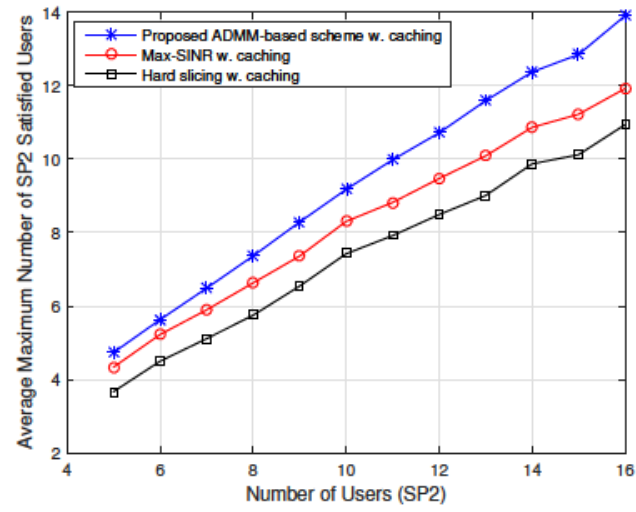

(c) SP 2

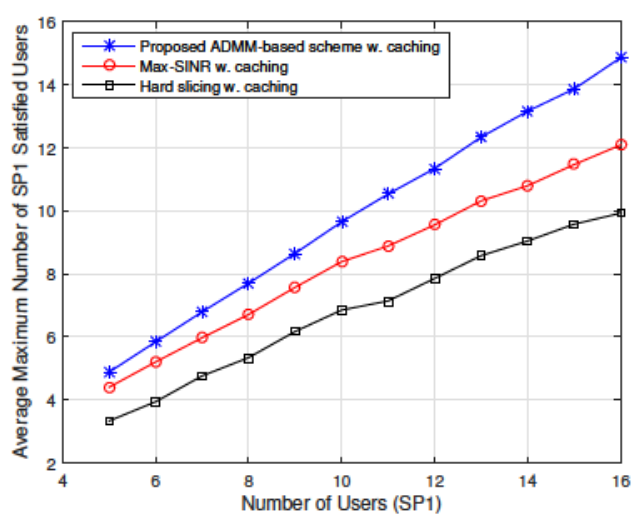

(b) SP 1

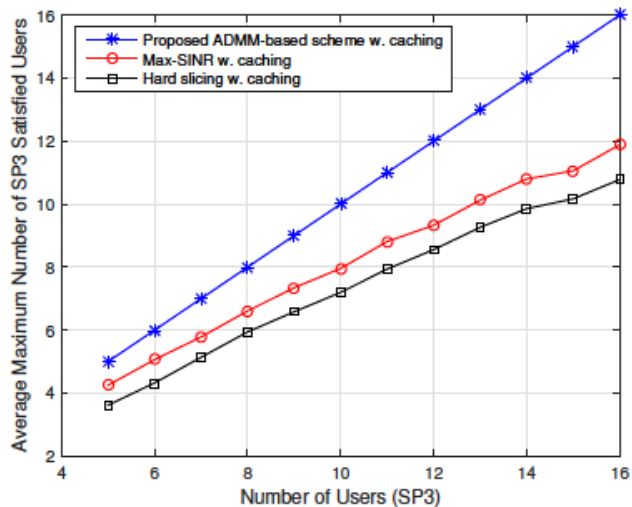

(d) SP 3

Figure 2.4: Comparison of the maximum number of satisfied users.

number of access users and the y-axis is the maximum number of satisfied users. The required data rate of each user is the same, which is $8 \mathrm{Mbps}$, and the number of users of each SP is also the same. From the figure, we can observe that our proposed scheme can almost satisfy all the users when the number of users is less than 40 . Even with more than 40 users, the performance of the ADMM-based scheme is better than the other two schemes. We also compare the satisfied users in each individual SP in Fig. 2.4b-2.4d. Similar observations can be made.

We also evaluate the effects of different required data rates. As shown in Fig. 2.5 , with the increase of the required data rate, the number of maximum satisfied 
users decreases for all three schemes. In the low data rate area, all three schemes can satisfy all access users. However, the performance has a significant fall when the data rate is higher than $12 \mathrm{Mbps}$, because the total radio resource is limited.

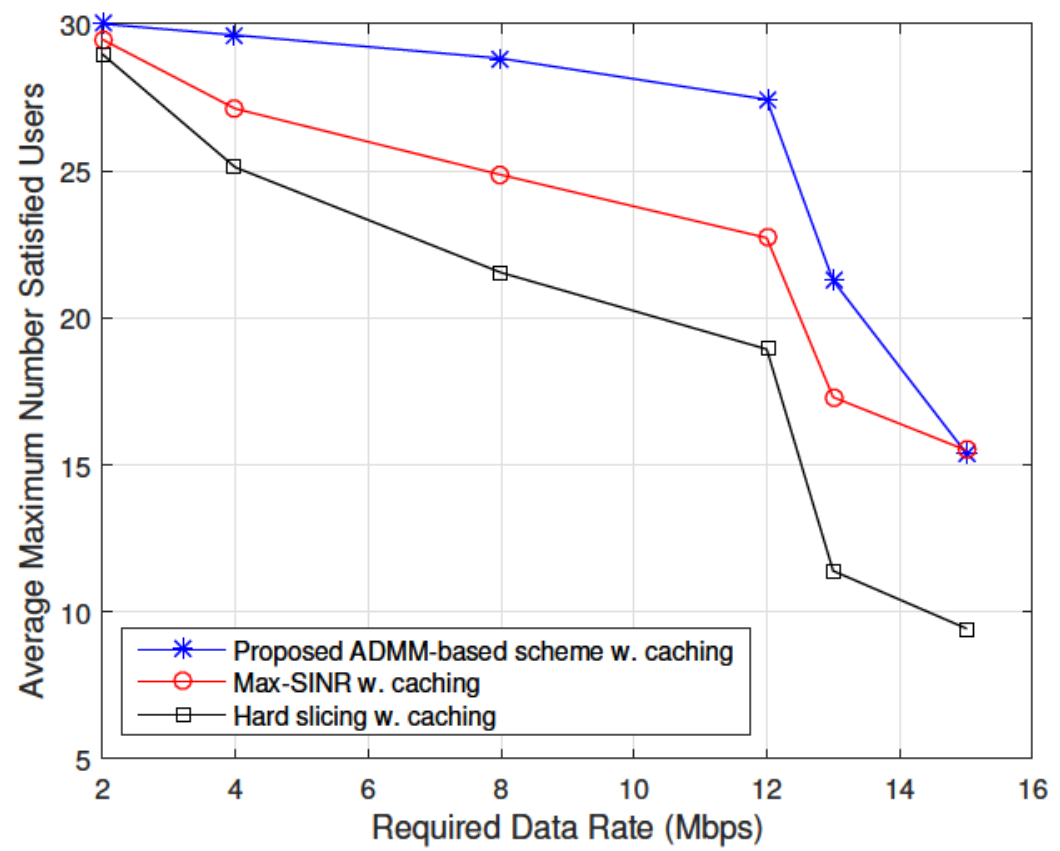

Figure 2.5: The effects of the required data rate. The number of users = 30.

\subsubsection{The Effects of Caching with Virtualization}

The gain of deploying in-network caching is evaluated next. Fig. 2.6 shows the average aggregated backhaul access costs used by all users. From Fig. 2.6, we can observe that all three schemes with in-network caching significantly reduce the total backhaul usage, compared to the cases without in-network caching. This is because the proposed information-centric wireless virtual network framework enables in-network caching, which reduces duplicate content transmissions in networks. It should be noted that the backhaul access costs of the ADMM-based scheme are higher than 


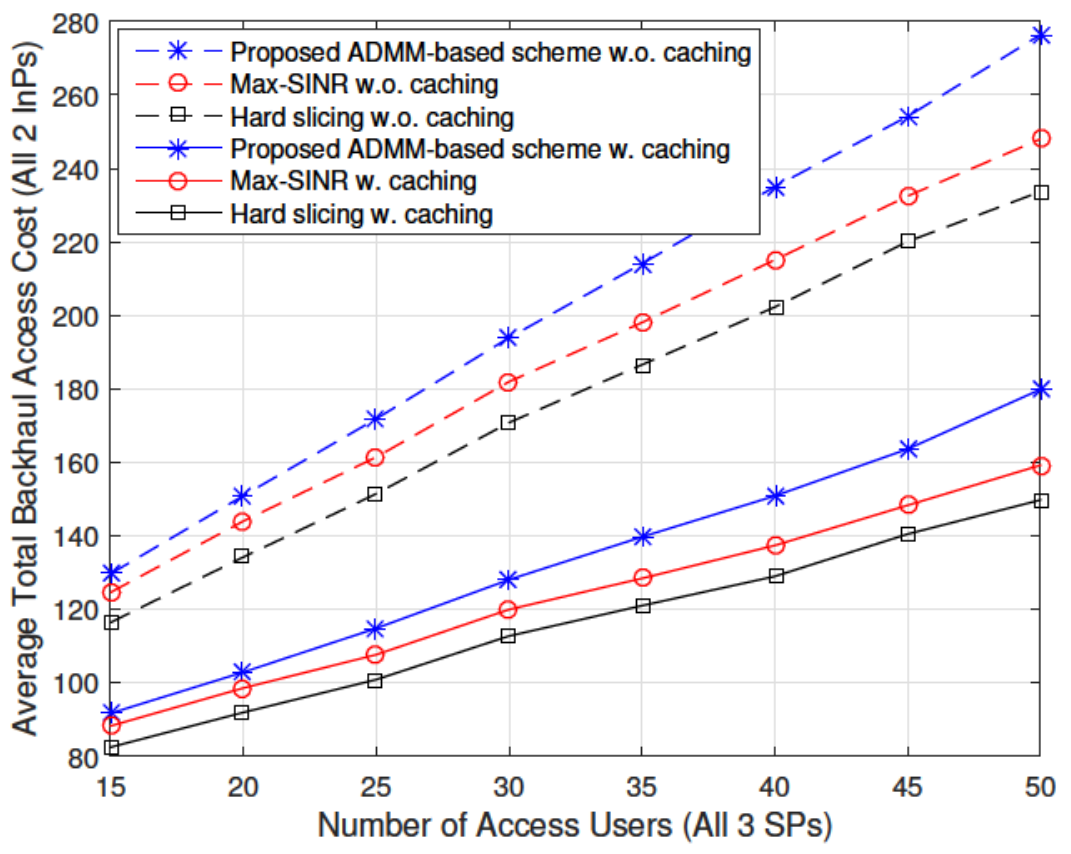

Figure 2.6: Comparison of the backhaul access costs.

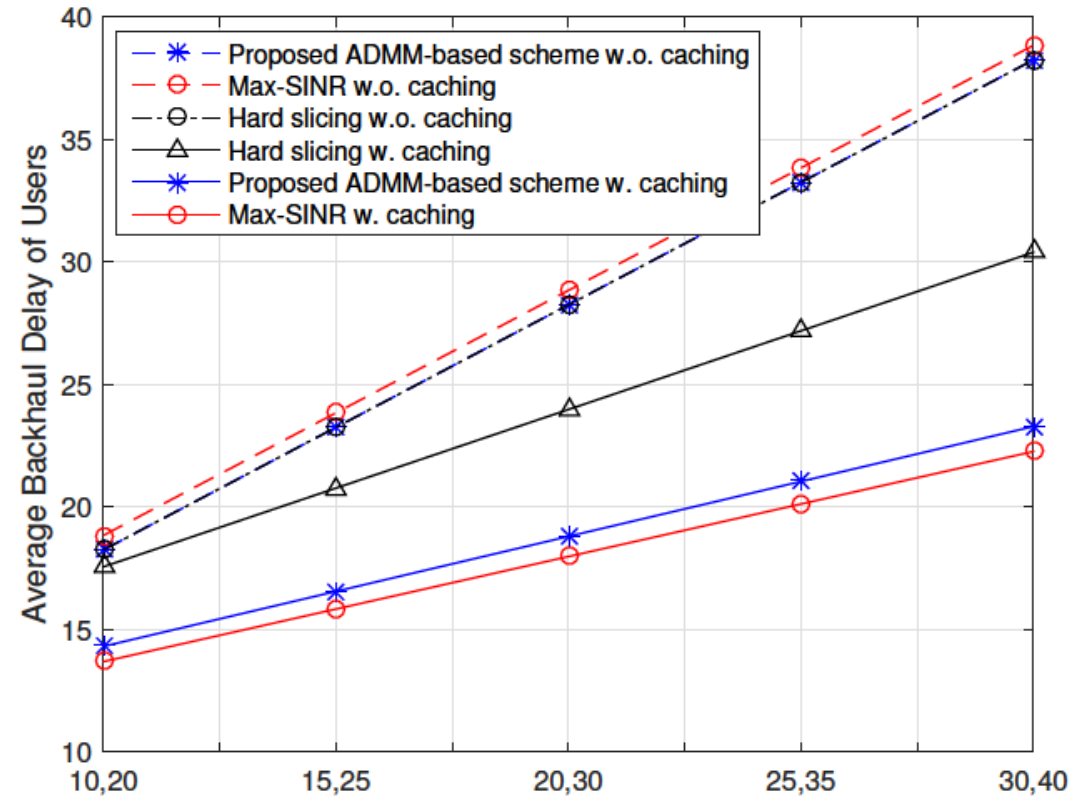

Fixed Backhual Network Delay (Macro Backhaul (ms), Femto Backhaul(ms))

Figure 2.7: Comparison of the average Delay. The number of users $=30$. 


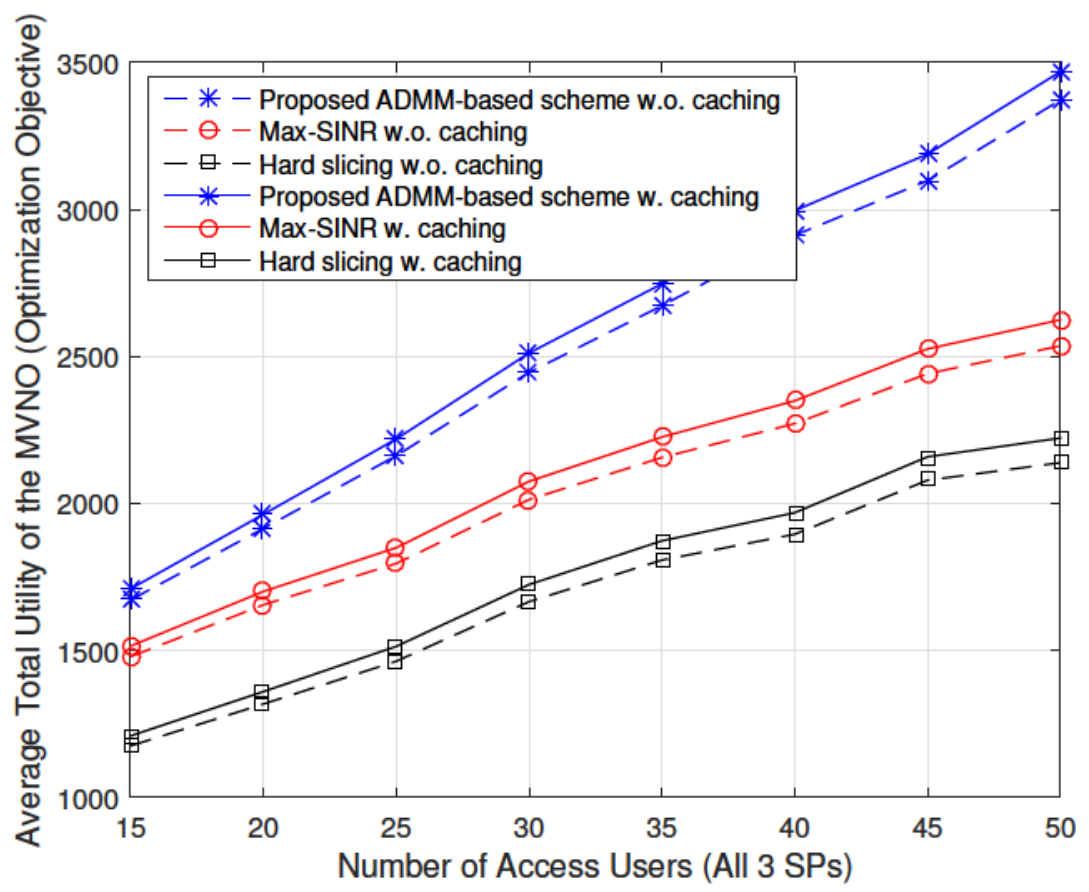

Figure 2.8: Comparison of the MVNO utility.

those of the other two schemes, because the ADMM-based scheme provides better access performance (more users and higher data rate).

Another important parameter to evaluate the performance is backhaul delay. In Fig. 2.7, with the increase of backhaul delay, the average delay of users is getting larger. However, by deploying in-networking caching, the delay is much lower than the cases without caching, and the increase of delay can be depressed.

Fig. 2.8 shows the total utility of the MVNO in different schemes. We can see that the utility of the MVNO increases with the increase of the number of users for all these cases. This is because more payment can be obtained from SPs with the increase of the number of users. From Fig. 2.8, we can observe that the proposed ADMM-based scheme gives better performance than the traditional max-SINR and the hard slicing virtualization scheme. Furthermore, by deploying in-network caching, the performance is better than that without caching, because caching can reduce 
backhaul access costs and lower the delay.

\subsection{Conclusions}

In this chapter, we jointly studied wireless network virtualization and informationcentric networking in next generation cellular networks. We proposed an informationcentric wireless network virtualization framework for enabling both wireless network virtualization and ICN. Then, we formulated the virtual resource allocation and innetwork caching strategy as an optimization problem, which maximizes the utility of mobile virtual network operators. In addition, we developed an efficient ADMM-based distributed virtual resource allocation and in-network caching scheme. Simulation results were presented to show that the performance of backhaul alleviation can be substantially improved in the proposed scheme with in-network caching. The InPs, SPs and MVNOs can benefit from the proposed information-centric wireless network virtualization framework. 


\section{Chapter 3}

\section{Robust Admission Control of VNs}

\subsection{Introduction}

While excellent works have improved the performance of wireless network virtualization, the QoS experienced by users in VNs can be significantly affected by virtualization, because virtual networks share the same underlaying physical network, and both the traffic and wireless channels can change dynamically [4].

To guarantee the QoS, admission control of end users has been studied extensively in traditional wireless networks (see $[89,90]$ and the references therein). By limiting the number of UEs entering the network, UE admission control can effectively guarantee QoS and maximize network utilization simultaneously. In this chapter, motivated by the experience of UE admission control in traditional wireless networks, we propose a novel concept of VN admission control for wireless virtualization. By limiting the number of VNs embedded in the physical network, VN admission control can effectively guarantee QoS experienced by users of VNs and maximize the utilization of the physical networks at the same time. To the best of our knowledge, VN admission control has not been well studied in the literature. The contributions of this chapter are as follows. 
- In addition to the physical resource leasing and allocation issues studied in previous works, we jointly study $\mathrm{VN}$ admission control with these issues for wireless virtualization. Specifically, we propose a two-stage VN embedding mechanism that can decouple short-term physical resource allocation from long-term admission control and resource leasing.

- With recent advances in robust optimization (RO) [91], we formulate the VN admission control problem as a robust optimization problem. RO is a novel technology in optimization theory that is suitable for solving problem with uncertain data [92]. RO has been successfully used in signal processing [93] and resource allocation [94,95], among others. In the $\mathrm{VN}$ admission control problem, uncertainty arises when estimating the capacity of underlaying physical networks and the traffic of VNs.

- Both the long-term admission control and short-term resource allocation problems are transformed to convex problems, which can be solved efficiently.

- An example of implementation is given to illustrate the procedure of our proposed scheme. Simulation results are presented to show the effectiveness of the proposed scheme.

The rest of this chapter is organized as follows. Section 3.2 introduces system model and describe the problem of VNs embedding. The proposed two-stage VNs admission mechanism is presented in Section 3.3. Section 3.4 discusses simulation results. Finally, we conclude this study in Section 3.5. 


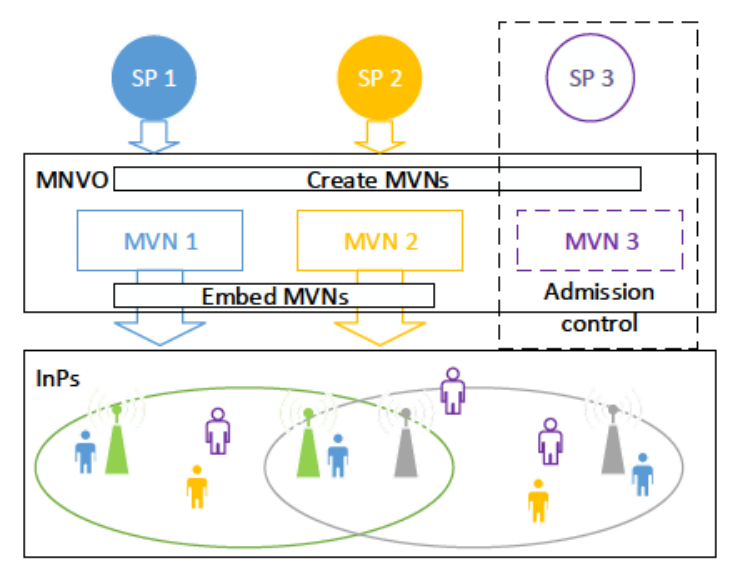

Figure 3.1: System model of the virtualized wireless network.

\subsection{System Model and Problem Statement}

In this chapter, the models of business roles virtual networks and the caching are the same as Chapter 2. In this section, we present differences and additional information of the system model. The proposed problem is formulated after related assumptions are given. Other notations different from Chapter 2 are summarized in Table 3.1.

\subsubsection{Network Model}

For example, shown as in Fig. 3.1, there are two InPs (green and grey), one MNVO, and three SPs. VNs requested from SP 1 and SP 2 have been embedded in the physical network. Meanwhile, SP 3 is requesting a VN to provide connections to its users (purple). the MNVO needs to make the admission control decision to decide whether or not admit this new MNV request based on current network status and SP 3's requirements. If this request is proved, the MVNO has to lease resource from InPs and embed this VN into physical networks.

Let us consider an area (e.g., a campus or a district) covered by several BSs operated by a set $\mathcal{J}$ of InPs. To simplify our analysis, we assume each $\operatorname{InP} j$ can fully 
Table 3.1: Notations of Chapter 3

\begin{tabular}{|c|c|}
\hline Notation & Definition \\
\hline $\mathcal{J}$ & the set of mobile BS nodes (e.g., MBSs, SBSs) \\
\hline $\mathcal{K}$ & the set of SPs \\
\hline $\mathcal{I}$ & the set of UEs \\
\hline$\alpha_{j, \max }$ & max radio resources \\
\hline$y_{j}$ & the borrowing ratio of $\operatorname{InP} j$ \\
\hline$\psi_{j}^{c}$ & the access cost of leasing network resource \\
\hline$f^{c}$ & total cost \\
\hline$\tilde{d}_{k}$ (bps) & the data rate requirement with mean value of $d_{k}$ \\
\hline$r_{i_{k}}, r_{i j}$ & the provisioned bandwidth \\
\hline$\beta_{j}$ & the scheduling policy of the mobile network \\
\hline$a_{k} \in\{0,1\}$ & the admission indicator of the $k$ th $\mathrm{VN}$ \\
\hline$\psi_{k}^{r}$ & admission revenue \\
\hline$\psi_{k}^{p}$ & blocking penalty \\
\hline$f^{u}$ & the total utility that MVNO can get from SPs \\
\hline$\lambda_{k}$ (users/second) & the arrival rate of the users in $\mathrm{VN} k$ \\
\hline $1 / \mu_{k}$ (seconds) & the session holding time of VN $k$ \\
\hline$F_{k}($ bits $)$ & file size \\
\hline$c_{j}$ (bps) & the capacity provided by $\operatorname{InP} j$ \\
\hline$R_{j}^{[t]}$ & the aggregated data rate provided by $\operatorname{InP} j$ at time $t$ \\
\hline$\rho$ & the total expected traffic load (request throughput) \\
\hline$B_{j}(\mathrm{~Hz})$ & the total spectrum bandwidth of BS $j$ \\
\hline$l_{j}(\mathrm{~Hz})$ & the backhaul capacity of BS $j$ \\
\hline$h_{i j}$ & the hitting event indicator \\
\hline$\mu, \pi, \delta, \alpha$, tau, sigma & parameters of robust optimization \\
\hline
\end{tabular}


cover the considered area by themselves. Total $\alpha_{j, \max } \leq 1$ resources of network can be used for virtualized networks created by the MVNO. Assume full network sharing is realized, the MVNO can borrow any physical network resource from all InPs. We use $y_{j} \in[0,1]$ to denote the borrowing ratio of $\operatorname{InP} j$ at the considered area. Thus,

$$
y_{j} \leq y_{j}^{\max }, \forall j .
$$

By denoting the access cost of leasing network resource from $\operatorname{InP} j$ as $\psi_{j}^{c}$ ( $c$ represents cost), the cost can be estimated as

$$
f^{c}=\sum_{j \in \mathcal{J}} y_{j} \psi_{j}^{c}
$$

We assume total $K$ SPs denoted by $k \in \mathcal{K}:=\{1,2, \ldots, K\}$ in our system model and each SP is served by a VN to provide services to this end users. Assume there are a set $\mathcal{I}_{k}$ of active users at the considered area served by SP $k$ and that each is denoted by $i_{k} . d_{k}$ (bps) denotes the average data rate requirements of SP $k$, which means if VN $k$ is admitted to be embedded in the considered area, following constraints needs to hold:

$$
r_{i_{k}} \geq d_{k}, \forall i_{k}
$$

It should be noted that the provisioned bandwidth $r_{i_{k}}$ mainly depends on the scheduling policy of the mobile network denoted by $\beta_{j}$.

For simple notation, we use the same notation for VNs serving SPs. For total $K$ VNs (one SP owns one VN), the MVNO creates $K$ VNs. These VNs request to access the network dynamically, which means embedding request. If the physical network can embed these request VNs at the considered area, MVNO will admit the access 
of these VNs. Let $a_{k} \in\{0,1\}$ be the admission indicator of the $k$ th VN. Formally, if the MVNO admits the $k$ th VN, $a_{k}=1$; if block, $a_{k}=0$.

$\psi_{k}^{r}$ is the weight parameter ( $r$ represents for admission revenue) for admitting the $k$-th VN and $\psi_{k}^{p}$ is the weight parameter ( $p$ represents blocking penalty) for blocking the $k$ th VN. Therefore, the utility that MVNO can get from SPs at any area is shown as follows:

$$
f^{u}=\sum_{k \in \mathcal{K}} f_{k}^{u}=\sum_{k \in \mathcal{K}}\left[a_{k} \psi_{k}^{r}-\left(1-a_{k}\right) \psi_{k}^{p}\right]=\sum_{k \in \mathcal{K}}\left[a_{k}\left(\psi_{k}^{r}+\psi_{k}^{p}\right)-\psi_{k}^{p}\right]
$$

To model the traffic of VNs, let the arrival rate of the users in VN $k$ associated with the considered area be Poisson and thus be denoted by $\lambda_{k}$ (unit in users/second). The session holding time of VN $k$ is denoted by $1 / \mu_{k}$ (unit in second). $1 / \mu_{k}$ is decided by data rate $d_{k}$ and file length $F_{k}$, which is $1 / \mu_{k}=F_{k} / d_{k}$. Since data rate $d_{k}$ and file length $F_{k}$ are only related to service type (e.g., video streaming and file downloading), we assume for all area $d_{k}$ and $F_{k}$ are the same. However, unlike holding time, the arrival rates are based on service type and varying with time. Thus, it is very hard to evaluate the arrival rate accurately. Following the research in [96], we define $\rho_{k}=F_{k} \lambda_{k}$ to be the mean traffic of VN $k$. Therefore, the total expected traffic load (request throughput) is

$$
\rho=\sum_{k}^{K} a_{k} \rho_{k}
$$

Unfortunately, in practical network virtualization, the real arrival rate $\lambda_{k}$ varies with time, which implies that the arrival rate of users given by a specific $\mathrm{VN}$ is an estimated mean value. Thus, the estimated arrival rate $\lambda_{k}$ is actually a nominal value and not accurate so that we have to tolerate this uncertain inaccuracy. To model the uncertainty, following studies in [96] and [97], we assume the actual arrival rate as a 
random variable $(\mathrm{RV})$ denoted by $\tilde{\lambda_{k}}$ with following features:

- $\tilde{\lambda_{k}}$ is with mean value of $\lambda_{k}$;

- $\tilde{\lambda}_{k}$ is bounded by $\left[\lambda_{k}^{l}, \lambda_{k}^{h}\right]$;

- $\tilde{\lambda_{k}}$ are independent of each other;

- $\lambda_{k}^{l}$ and $\lambda_{k}^{h}$ are known in advanced.

$\lambda_{k}^{l}$ and $\lambda_{k}^{h}$ are the lowest and highest arrival rates of $\mathrm{VN} k$ and depend on the type (e.g., a large network or a small group of users) and timing (e.g., rush hours or night) of the VN, which can be obtained by historical statistic. Since VNs are isolated with each other, $\tilde{\lambda_{k}}$ are uncorrelated. Therefore, (3.5) is revised as:

$$
\rho=\sum_{k}^{K} a_{k} F_{k} \tilde{\lambda_{k}}
$$

Noting that the leased physical resources from InP $j$ used for embedding VNs is $y_{j}$, we thus use $c_{j}$ (bps) to denote the capacity provided by $\operatorname{InP} j$. Obviously, $c_{j}$ is decided by current distribution of users (e.g., positions and mobility) and the physical resource allocation policy. Practically, $c_{j}$ is varying with time and locations, thus we can obtain an estimated $c_{j}$ based on historical data and current network status. An method proposed in [67] for virtualized LTE networks is adopted in this chapter. Assuming the aggregated data rate provided by one $\operatorname{InP} j$ at time $t$ is $R_{j}^{[t]}$, our expected capacity is

$$
c_{j}^{[t+1]}=\eta R_{j}^{[t]}+(1-\eta) c_{j}^{[t]}
$$

where $\eta \in[0,1]$ is a constant to adjust the ratio of current network status and previous network status. It should be noted that $R_{j}^{[t]}$ is a function of $y_{j}$ and the scheduling 
policy of radio resource (e.g., the cell association, the spectrum and power allocation) denoted by $\beta_{j}$. We assume the set of all available scheduling strategies is $\mathcal{Y}$ in this study.

Therefore, with the capacity and the traffic in hand, similar with [96], the system is stable only if

$$
\frac{\sum_{k}^{K} a_{k} F_{k} \tilde{\lambda_{k}}}{\sum_{j \in \mathcal{J}} y_{j} c_{j}} \leq 1
$$

This stable condition is the key constraint in our optimization problem.

\subsubsection{Problem Statement}

The MVNO is trying to maximize the utility $f^{u}$, thus we can obtain the following optimization problem:

$$
\begin{aligned}
\max _{\substack{a_{k} \in\{0,1\}, y_{j} \in \Re^{+}, \beta_{j} \in \mathcal{Y}}} & f^{u}-f^{c} \\
\text { s.t. } & (3.1),(3.3),(3.8) .
\end{aligned}
$$

In the above problem (3.9), it is difficult to solve the three types of variables $a_{k}$, $y_{j}$, and $\beta_{j}$ at the same time due to different time scales and traffic realizations. $a_{k}$ used to control the embedding decision of VNs are in large time scale (minutes to hours) and are decided before traffic realization. This is because a typical SP usually requests a $\mathrm{VN}$ for a period of time instead of a short moment. For instance, a sports game broadcast virtual network will stay in the network for hours, and a real time video conferencing network may exist for minutes. If the SP is operating a VoIP VN, it may ask a VN for days or months. This means $x_{k}$ should be optimized before users arrival with uncertain arrival rate. Similar to admission control, $y_{j}$ that can be considered as coordinative parameters among InPs should be optimized in large time 


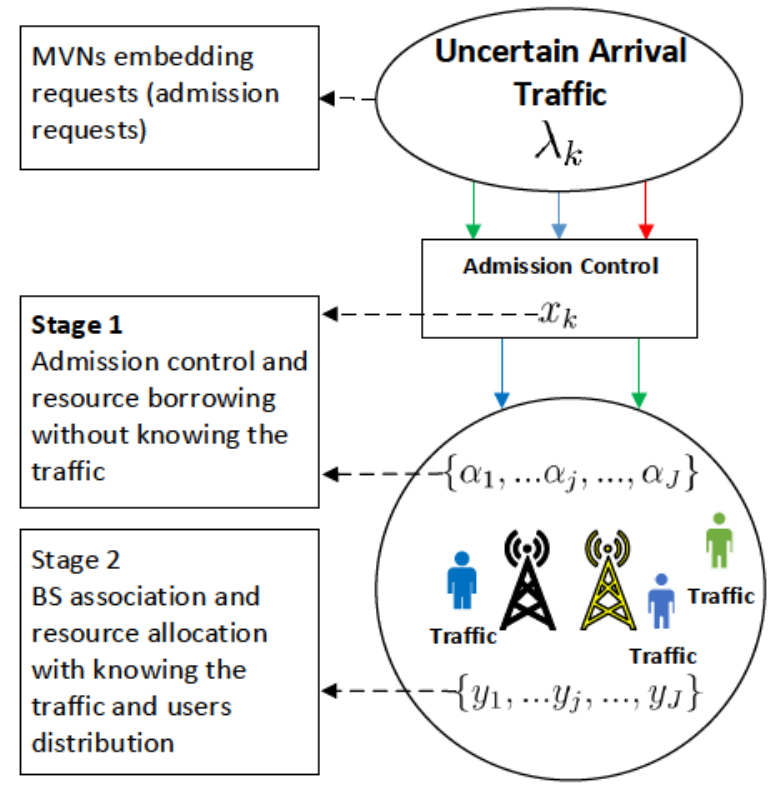

Figure 3.2: The two-stage VN embedding mechanism including admission control, resource borrowing and resource allocation.

scale and before traffic realization, because the MVNO should reserve enough resource for embedding VNs and also reduce the overhead of coordinating InPs. However, $\beta_{j}$ have to be optimized after user actually arrival and waiting for scheduling (traffic load uncertainty realization), which means $\beta_{j}$ is changing in ms to second (e.g., time slots). Thus, to address these unmatched optimization appropriately, we divide optimization problem (3.9) into two stages: 1) joint admission control and leasing optimization and 2) physical resource allocation optimization. This two-stage mechanism is described in Fig. 3.2.

In the first stage, without knowing the exact traffic load request from SPs, the MVNO conducts admission control and resource leasing based on the estimated arrival traffic $\tilde{\lambda_{k}}$ that are uncertain and provided by VNs. Obviously, in real networks, $\tilde{\lambda_{k}}$ varies with different long term time slots (e.g., rush hours and evening). Therefore, in the first stage, the system has to be able to tolerate this variance. 
In the second stage, the MVNO will perform a bandwidth provisioning. This stage can be considered as an extended stage of first stage after traffic arriving. As we consider multiple InPs in our model, this bandwidth provisioning includes radio resource allocation and BS association. In next subsection, we develop two schemes to solve these two problems.

\subsection{Proposed Scheme}

In this section, we propose a novel scheme to control the admission of new requests from VNs by deploying the robust optimization technique.

\subsubsection{Robust Admission Control and Resource Leasing}

Based on the above analysis, admission indicators $a_{k}$ and infrastructure leasing ratio $y_{j}$ are optimized before the realization of uncertainty $\lambda_{k}$. Since the resource allocation ratio $\beta_{j}$ is not included in the objective function, we only need to reform our constraints related to uncertainty instead of certain problem. Firstly, as $a_{k}$ are binary variable, problem (3.9) is a mixed integer linear problem (MINLP). Moreover, in addition to MILP that is not a easy problem to solve, RVs of parameters make the proposed problem uncertain.

For tractable solution of it, we firstly, relax the binary variables $a_{k}$ to real value $\hat{a}_{k} \in[0,1]$ so that problem (3.9) becomes a linear form. $\hat{a}_{k}$ can be interpreted as partial admission decision of $a_{k}$, which means the physical network cannot embed the whole VN's request but part of it. In long term view, $\hat{a}_{k}$ can be considered as the average time when $\mathrm{VN} k$ is embedded in the physical network. We obtain the first 
stage (optimizes $\hat{a}_{k}$ and $y_{j}$ ) of problem (3.9) formally formed as:

$$
\begin{aligned}
\max _{\hat{a}_{k}, y_{j} \in \Re^{+}} & f^{u}-f^{c}, \\
\text { s.t. } & (3.1), \hat{a}_{k} \leq 1, \forall k \\
& \sum_{k}^{K} \hat{a}_{k} F_{k} \tilde{\lambda}_{k}-\sum_{j \in \mathcal{J}} y_{j} c_{j} \leq 1 .
\end{aligned}
$$

Note that problem (3.10) is a linear problem if $\tilde{\lambda_{k}}=\lambda_{k}$ is fixed (certain). Since $\tilde{\lambda_{k}}$ is a random variable, it is very hard to guarantee the reserved (leasing) resource enough for $\tilde{\lambda_{k}}$ all the time, even impossible in real time system due to outdated information. Thus, instead, we may restrict the violence of the stability (the constraint [3.10b]) to a tolerable level (certain probability), namely chance-constraint. This 'soft' restriction is more efficient than that holds the constraints all the time because some peak values are reached only with a low probability. If we assume the probability that the the stability can be violated is $\varepsilon$, the 'chance' stability constraint can be defined as follows:

$$
\operatorname{Pr}\left\{\sum_{k}^{K} \hat{a}_{k} F_{k} \tilde{\lambda_{k}}-\sum_{j \in \mathcal{J}} y_{j} c_{j} \leq 1\right\} \geq 1-\varepsilon
$$

where $\operatorname{Pr}(x \leq X)$ means the probability of that $x$ is smaller than $X$.

Replacing (3.10b) by (3.11) , problem (3.10) becomes a chance-constrained problem that is a typical robust optimization problem [98]. The chance constraint (3.11) makes the optimization problem (3.10) significantly not tractable due to the uncertain feasible set supported by (3.11). If $\tilde{\lambda_{k}}$ has a nice distribution (e.g., symmetric logarithmically concave density [97,99]), (3.11) can support a convex feasible set. Nevertheless, it is hard to evaluate this. Thus, in this dissertation, we deploy Bernstein approximation to formulate a safe approximation to (3.11). Firstly, we define 
constants $\pi_{k}^{l}=\frac{1}{2}\left(\lambda_{k}^{h}-\lambda_{k}^{l}\right)$ and $\pi_{k}^{h}=\frac{1}{2}\left(\lambda_{k}^{h}+\lambda_{k}^{l}\right)$ so that

$$
\delta_{k}=\frac{\tilde{\lambda_{k}}-\pi_{k}^{h}}{\pi_{k}^{l}} \in[-1,1]
$$

Then, let $f_{0}\left(\hat{a}_{k}\right)=\sum_{k \in \mathcal{K}} \pi_{k}^{h} F_{k} \hat{a}_{k}-\left(\sum_{j \in \mathcal{J}} y_{j} c_{j}+1\right)$ and $f_{k}\left(\hat{a}_{k}\right)=\pi_{k}^{l} F_{k} \hat{a}_{k}, \forall j$, so that we have the equivalent expression of (3.11) shown as follows

$$
\operatorname{Pr}\left\{f_{0}\left(\hat{a}_{k}\right)+\sum_{k \in \mathcal{K}} \delta_{k} f_{k}\left(\hat{a}_{k}\right) \leq 0\right\} \geq 1-\varepsilon
$$

Observe that

- $\delta_{k}$ is bounded and supported by $[-1,1]$;

- $\delta_{k}$ are independent of each other;

- $f_{k}\left(\hat{a}_{k}\right)$ is an affine function of $\hat{a}_{k}$.

Holding these properties and following the research in [97,99], we can approximate (3.13) to a convex form as follows

$$
f_{0}\left(\hat{a}_{k}\right)+\sum_{k \in \mathcal{K}} \max \left\{\mu_{k}^{+} f_{k}, \mu_{k}^{-} f_{k}\right\}+\left(2 \log \frac{1}{\varepsilon}\right)^{\frac{1}{2}}\left[\sum_{k \in \mathcal{K}}\left(\sigma_{k} \pi_{k}^{l} \hat{a}_{k}\right)^{2}\right]^{\frac{1}{2}} \leq 0
$$

where $-1 \leq \mu_{k}^{-} \leq \mu_{k}^{+} \leq 1$ and $\sigma_{k}>0$ are constants that depend on the given probability distribution. Detailed information about Bernstein approximation and its conservatism can be found in $[98,99]$. In this study, based on the properties of $\delta_{k}$, we set $\mu_{k}^{+}=1 / 2$ and $\sigma_{k}=\sqrt{1 / 12}$.

Thus, after using Bernstein approximation to approximate (3.13) in problem 
(3.10), we can get the approximate problem shown as:

$$
\begin{aligned}
\max _{\substack{\hat{a}_{k} \in\{0,1\}, y_{j} \in \Re+}} & f^{u}-f^{c} \\
\text { s.t. } & (3.10 a),(3.14) .
\end{aligned}
$$

Obviously, problem (3.15) is a convex problem as both the objective function and constraints are either in linear or quadratic form. Thus, general convex solvers (e.g., CVX [100]) can be used to gain an optimum solution with effortlessness.

It should note that $\hat{a}_{k}$ is a real value bounded on $[0,1]$ that represents the partially admitting and the solution of the upper bound. However, if the partial admission is not allowed in the network, we have to recover $a_{k}$ from $\hat{a}_{k}$. There are many methods to round up real value to integer, such as arbitrary selection [83] and marginal benefit [101]. Unfortunately, due to the uncertainty of our optimization parameters that leads inaccurate solution, in this study, we develop a rounding method described as follows.

By solving problem (3.15), a upper bound solution $y_{j}^{*}$ of the leasing resource $y_{j}$ can be obtained. We can consider this solution as the maximum resource that are available to embed VNs. Thus, we needs to select a set of VNs that can utilize our resource the most efficiently, namely, providing the most utility. As $y_{j}^{*}$ is fixed, the selection of VNs only depends on $f_{k}^{u}$. Firstly, let us make all $a_{k}=0$ and sort VNs by their gradient $\left(\psi_{k}^{r}+\psi_{k}^{p}\right)$ from high to low. Then, we select total $K^{+}$VNs that give the highest gradients from $\mathcal{K}$ and let $a_{k}=1, \forall k \leq K^{+}$. In order to get $K^{+}$, we can give a following rule. $K^{+}$is the value that makes constraint (3.14) violated but (3.14) holds when $a_{k}=1, \forall k \leq K^{+}-1$.

It should be noted that, based on $y_{j}$ and $K^{+}$, the gap to upper bound is bounded by $\psi_{K^{+}}^{p}$ because the worst case is that $\hat{a}_{K^{+}}$is very near one. To narrow this gap, we 
can resolve the following problem by fixing all $a_{k}$ to drop some leased resources.

$$
\begin{aligned}
\max _{y_{j} \in \Re^{+}} & f^{u}-f^{c} \\
\text { s.t. } & (3.10 a),(3.14) .
\end{aligned}
$$

Problem (3.16) is a linear problem that can be solved easily.

\subsubsection{Robust Bandwidth Provisioning}

In this subsection, to solve the second stage, we need to form a bandwidth provisioning problem to reserve resource for users in the admitted VNs.

Firstly, we need to present the users who are admitted to the network and waiting for scheduling. If we use $\mathcal{K}^{+}$to indicate the set of VNs that have been admitted to be embedded in the considered area, we can get a set of active users shown as follows:

$$
\mathcal{I}:=\left\{i \mid \forall i_{k} \in \mathcal{I}_{k}, k \in \mathcal{K}^{+}\right\}
$$

To provision enough bandwidth for users, we have to consider the uncertainty of the coming traffic as well. Thus, similar to the arrival rate $\lambda_{k}$, we assume the data rate of user $i$ is a random variable $\tilde{d}_{i}$ with following features:

- $\tilde{d}_{i}$ is with mean value of $d_{i}=d_{k}$ where $d_{k}$ is the requirements of SP $k$;

- $\tilde{d}_{i}$ is bounded by $\left[d_{i}^{l}, d_{i}^{h}\right]$ where $d_{i}^{l}$ and $d_{i}^{h}$ are the lowest data rate and peak rate of the demand of user $i$;

- $\left\{\tilde{d}_{i}\right\}$ are independent of each other;

- $d_{i}^{l}$ and $d_{i}^{h}$ are known in advanced.

Those assumptions are reasonable in real networks and similar to [97], which makes the problem solvable. $d_{i}^{l}$ and $d_{i}^{h}$ depend on the type of user applications and since 
the content is randomly selected, $\left\{\tilde{d}_{i}\right\}$ are uncorrelated. The information of $d_{i}^{l}$ and $d_{i}^{h}$ can be stored at cache or delivered to the SDN controller by the control plane. In reality, $d_{i}^{l}$ and $d_{i}^{h}$ can be various from applications.

Meanwhile, we assume that each flow of user $i$ can be split to multiple paths and delivered by different BSs. The bandwidth provisioned by BS $j$ is denoted by $r_{i j}$ (bps). Obviously,

$$
r_{i_{k}}=r_{i j}
$$

For any BS $j$, we assume that the backhaul consumption (bandwidth) is equal to the total data rate of all flows that the BS $j$ needs to bear, only expect that the content is in the cache of node $j$, namely the hitting event indicator $h_{i j}=1$. For any $\operatorname{BS} j$, the reserved bandwidth $R_{i j}$ for user $i$. Thus, let $\bar{h}=1-h$, we have

$$
\sum_{i \in \mathcal{I}} \bar{h}_{i j} r_{i j} \leq l_{j}, \forall j \in \mathcal{J}
$$

where $l_{j}$ is the maximum capacity of the backhaul.

Since the spectrum bandwidth of each BS is limited by $y_{j}^{*} B_{j}$, the following constraints has to hold

$$
\sum_{i} \frac{r_{i j}}{\gamma_{i j}} \leq y_{j}^{*} B_{j}, \forall j
$$

As mentioned above, this subsection aims to provide an efficient mechanism to improve the bandwidth provisioning of VNs under uncertain traffic while utilizes the in-network cache. First we introduce a set of arbitrary variables $\left\{\omega_{i j}\right\}$ representing the ratio of user $i$ carried by BS $j$. The introduction of $\left\{\omega_{i j}\right\}$ can help us to utilize robust optimization technique and practically can evaluate the traffic load of each 
node. If we assume the probability that the required data rate can be violated is $\varepsilon$, the QoS constraints can be defined as follows:

$$
\operatorname{Pr}\left\{\sum_{j \in \mathcal{J}} \omega_{i j} \tilde{d}_{i} \leq \sum_{j \in \mathcal{J}} r_{i j}\right\} \geq 1-\varepsilon, \forall i
$$

Moreover, to maximize the total throughput of the considered area, we define our problem as follows

$$
\begin{aligned}
\max _{\substack{r_{i j} \in \mathbb{R}^{+} \\
\omega_{i j} \in \mathbb{R}^{++}}} & \sum_{i \in \mathcal{I}, j \in \mathcal{J}} \omega_{i j} \\
\text { s.t. } & \sum_{j} \omega_{i j} \geq 1, \forall i . \\
& (3.19),(3.20),(3.21),
\end{aligned}
$$

Obviously, problem (3.22) becomes a typical robust optimization problem as well. Firstly, we define constants $\alpha_{i}^{l}=\frac{1}{2}\left(d_{i}^{h}-d_{i}^{l}\right)$ and $\alpha_{i}^{h}=\frac{1}{2}\left(d_{i}^{h}+d_{i}^{l}\right)$ so that

$$
\tau_{i}=\frac{\tilde{d}_{i}-\alpha_{i}^{h}}{\alpha_{i}^{l}} \in[-1,1]
$$

Then, let $g_{0}\left(\omega_{i j}\right)=\sum_{j} \alpha_{i}^{h} \omega_{i j}-\sum_{j}\left(r_{i j}-\sum_{\substack{k \in \mathcal{J} \\ k \neq j}} \bar{h}_{i k} r_{i k}\right)$ and $g_{j}\left(\omega_{i j}\right)=\alpha_{i}^{l} \omega_{i j}, \forall j$, so that we have the equivalent expression of (3.21) shown as follows

$$
\operatorname{Pr}\left\{g_{0}\left(\omega_{i j}\right)+\sum_{j} \tau_{i} f_{j}\left(\omega_{i j}\right) \leq 0\right\} \geq 1-\varepsilon
$$

Observe that

- $\tau_{i}$ is bounded and supported by $[-1,1]$;

- $\left\{\tau_{i}\right\}$ are independent of each other; 
- $g_{j}\left(\omega_{i j}\right)$ is affine function of $\omega_{i j}$.

Holding these properties and following the research in [97, 99], we can approximate (3.23) to a convex form as follows

$$
g_{0}\left(\omega_{i j}\right)+\sum_{j} \max \left\{\mu_{j}^{+} f_{j}, \mu_{j}^{-} f_{j}\right\}+\left(2 \log \frac{1}{\varepsilon}\right)^{\frac{1}{2}}\left[\sum_{j}\left(\sigma_{j} \alpha_{i}^{l} \omega_{i j}\right)^{2}\right]^{\frac{1}{2}} \leq 0
$$

where $-1 \leq \mu_{j}^{-} \leq \mu_{j}^{+} \leq 1$ and $\sigma_{j}>0$ are constants that depend on the given probability distribution. Detailed information about Bernstein approximation and its conservatism can be found in $[98,99]$. Similar to above subsection, based on the properties of $\tau_{i}$, we set $\mu_{j}^{+}=1 / 2$ and $\sigma_{j}=\sqrt{1 / 12}$.

Thus, after using Bernstein approximation to approximate (3.21) in problem P0, we can get the approximate problem shown as:

$$
\begin{aligned}
\max _{\substack{R_{i j} \in \mathbb{R}^{+} \\
\omega_{i j} \in \mathbb{R}^{++}}} & \sum_{i \in \mathcal{I}, j \in \mathcal{J}} \omega_{i j} \\
\text { s.t. } & \sum_{j} \omega_{i j} \geq 1, \forall i . \\
& (3.19),(3.20),(3.24),
\end{aligned}
$$

Obviously, problem (3.25) is a convex problem as both the objective function and constraints are either in linear or convex form. Thus, general convex solvers (e.g., CVX [100]) can be used to gain an optimum solution with effortlessness.

\subsection{Simulation Results and Discussions}

In the simulations, we consider three LTE-based RAN InPs with $20 \mathrm{MHz}$ licensed spectrum. The locations of the macro BSs are fixed in the center of each cell with a 
coverage 250 meters representing an urban environment. Transmit power of $49 \mathrm{dBm}$ is set for macro BSs who have $20 \mathrm{MHz}$ spectrum respectively [86]. Referred to [81], we user a path loss $L(d)=34+40 \log (d)$ to model the macro cell propagation. The log-normal shadowing with standard deviation $8 \mathrm{~dB}$ for macro cell is assumed in our simulation. The power density of thermal noise power is $-174 \mathrm{dBm} / \mathrm{Hz}$ [86]. The unit leasing price price set by these three RAN InPs are all 1unit/MHz. One MVNO is assumed to provided VNs to maximum 10 SPs. The unit access price of one VN is set to be 2 units and blocking penalty is set to be 1 unit/VN. The traffic model assumed in our simulation is similar to [96]. The average file length is $40 \mathrm{Mbs}$. The average users arrival rates of any VN are expand from 0.4 users/second to 0.8 users/second, which means total arrival rates are 4 users/second to 8 users/second. The uncertainty of arrival rates is maximum $20 \%$. The requirement of VNs is 0.05 , which means that at most $5 \%$ users are not satisfied with their data rate requests is acceptable. The data rate request for each user is $100 \mathrm{kbps}$. Moreover, we assume the arrival rate of VNs $\left(\lambda_{k}\right)$ ranges from $2 \mathrm{VNs} /$ hour to $3 \mathrm{VNs} /$ hour and depart rate $\left(\mu_{n}\right)$ is $1 \mathrm{VN} /$ hour. They both follow Poisson distribution.

To compare our proposed algorithm, two benchmarks without using $\mathrm{RO}$ are also considered. The first scheme, named fixed arrival rate $A C$ (fixed-AC), operates admission control without considering the uncertainty of arrival rate. It only controls the access of VNs based on average users arrival rate. Unlike first scheme, the second scheme, named max arrival rate $A C$ (max-AC), considers the maximum uncertainty when performing admission control, which means they always use at least $(1+\epsilon \lambda)$ as the arrival rate. For simple notation, our proposed two-stage VN embedding mechanism is denoted by robust optimization admission control (ROAC).

In simulations, we select three metrics to verify our proposed scheme. They are blocking probability of VNs, reserved physical resource and number of satisfied users. 
In the first simulation, we evaluate the effect of the $\mathrm{VN}$ arrival rate and users arrival rate on the blocking probability of VNs. In Fig. 3.3, the dotted lines represent user arrival rate $\lambda_{k}=6$ users $/ \mathrm{s}$ and the solid lines represent user arrival rate $\lambda_{k}=4$ users $/ \mathrm{s}$. The $\mathrm{x}$-axis is the arrival rate of VNs. As shown in Fig. 3.3, increases of both VNs arrival rate (2 to 3 ) and users arrival rate ( 4 and 6 ) higher the blocking probability of VNs. Firstly, higher users arrival rate leads that more resources are needed for each VNs. This results in less position of potential accessed VNs which affects the blocking probability directly. Secondly, according to the property of Poisson distribution, block probability increases with the increase of arrival rate of VNs as the network load $\rho_{k}=\lambda_{k} / \mu_{k}>1$. From Fig. 3.3, we can see that the max-AC scheme blocks more VNs. This is because the max-AC scheme has the least accessible VNs, and the max-AC scheme has to always consider the maximum users arrival rate. Meanwhile, if the admission control scheme (shown by fixed-AC) does not consider the uncertainty in arrival rate, it will allow the most VNs to access. Obviously, our proposed scheme shows a balance between max-AC and fixed-AC. Since ROAC takes uncertainty into consideration, it shows more cautious about admitting VNs leading appropriate number of positions of VNs. To evaluate the benefits from this balance, in the second simulation shown in Fig. 3.4, we use a metric to evaluate the average resource needed for one VN. This metric is called normalized reserved resource ratio, which is defined as reserved resource / total resource / number of admitted VNs. In Fig. 3.4, we can see that ROAC borrows more physical resource from InPs than fixed-AC, because it allows robustness brought by uncertainty. Compared to max$\mathrm{AC}, \mathrm{ROAC}$ can save more resource as max-AC puts the maximum traffic load into optimization. Obviously, ROAC gives a balance solution between admission control and resource leasing.

In the third simulation, we evaluate the QoS performance of our proposed ROAC. 


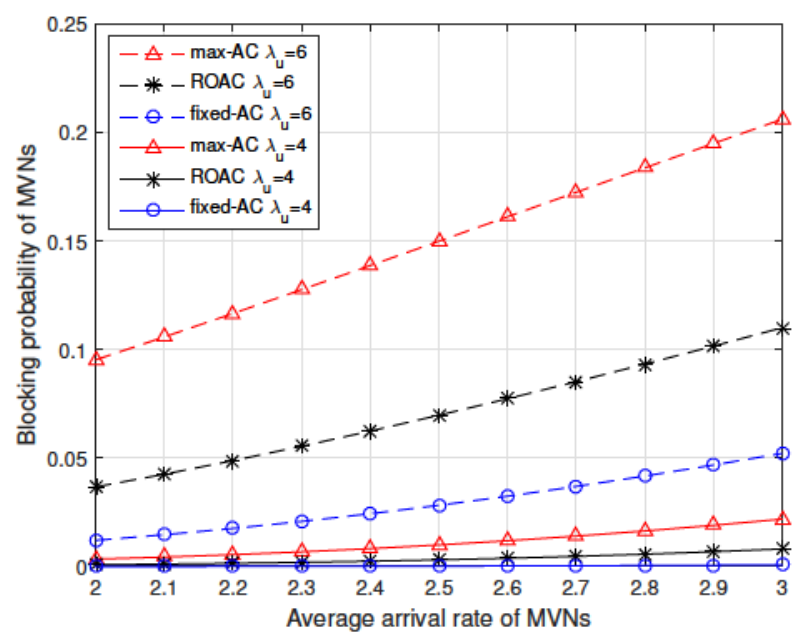

Figure 3.3: The effect of user arrival rate in VNs on the blocking probability of VNs.

Satisfied ratio (a ratio between the number of users served by required data rate to the total number of users) is used to verify QoS performance. Fig. 3.5 shows that both max-AC and ROAC guarantee the QoS requirement (satisfied ratio), which is $95 \%$. For max-AC, as it considers the maximum traffic when performs AC, it has less users in the network. Thus, almost every user can be served with full requirement. Our proposed ROAC scheme cannot satisfy all the users because we allow a probability (QoS requirement) that the system is not stable in our RO model. The worst case is given by the fixed arrival rate $\mathrm{AC}$, because it allows too much VNs without considering the uncertainty of arrival rate that directly affects the traffic in the network.

In summary, the proposed two-stage VN embedding scheme gives better QoS performance than the AC schemes that do not consider the uncertainty of traffic. Meanwhile, it can save more resources than the scheme that always takes the maximum traffic when optimizing the performance of networks.

To evaluate our proposed robust bandwidth provisioning, 3 schemes of provisioning bandwidth in SDWNs are considered. Scheme 1 is a centralized jointly radio resource allocation with backhaul limitation without considering time varying flows 


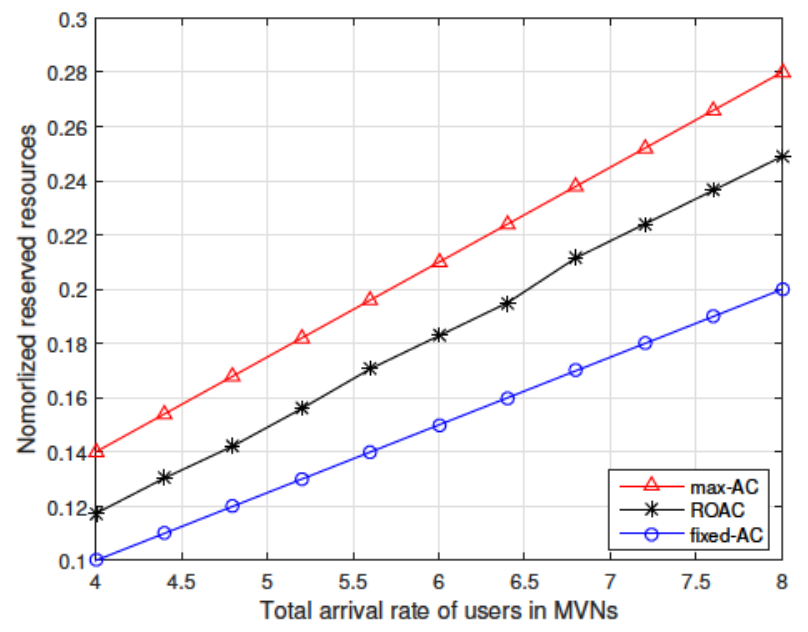

Figure 3.4: The effect of user arrival rate in VNs on the reserved physical resource.

rate and in-network caches. Scheme 2 is our proposed schemes considering in-network cache but without deploying robust optimization. Scheme 3 is our proposed schemes utilizing in-network cache and deploying robust optimization. The maximum number of BSs that each user can associate is set to 1 (single association). Average data rate demand of each flow is randomly selected from [25, 12, 10, 5, 2, 1.5] Mbps, and the peak-rate is maximum $150 \%$ of the selected average rate. Moreover, the tolerance level $\epsilon$ is set to be 0.2 that means we restrict the probability of violating the $\mathrm{QoE}$ requirement at most to be 0.2 .

To evaluate the effectiveness of robust optimization technique, in the first test, we change the peak to mean ratio of from $0.2,0.5$ to 1 , which means that the actual flow data rate can reach up to $120 \%, 150 \%$ and $200 \%$ of the average rate, respectively. We calculate the average number of flows, whose allocated bandwidth are larger than their real-time flow rate, for Scheme 1 and Scheme 2. As shown in Fig. 3.6, with robust optimization, more flows (3 flows) are allocated adequate bandwidth compared to where no robust optimization. However, with increasing peak-rate, the satisfied flows are decreasing in both two situations. 


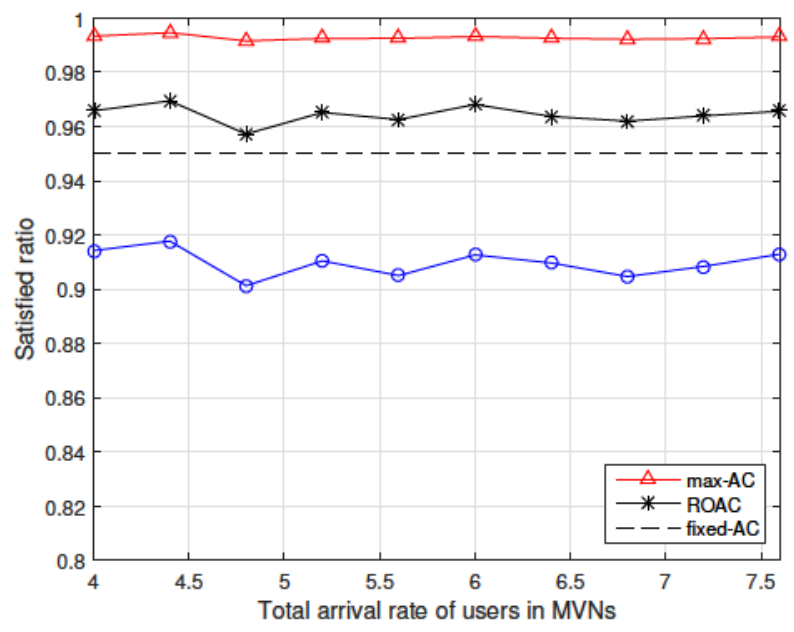

Figure 3.5: Performance of different admission control schemes.

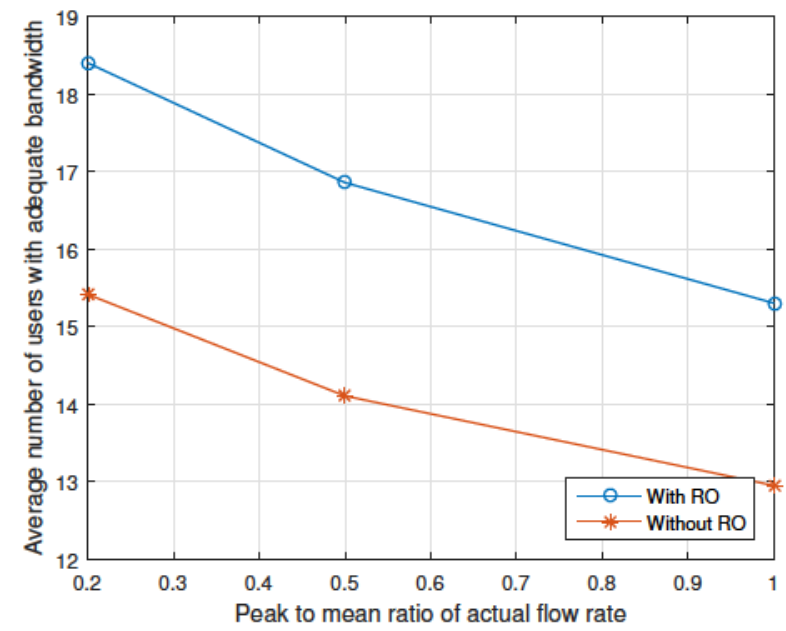

Figure 3.6: Effects of varying flows rate on the bandwidth provisioning.

In our next evaluation, we test the effectiveness of in-network cache and robust optimization technique. We defined a metric call normalized relative bandwidth (NRB) that is the ratio of relative provisioning bandwidth (provisioned bandwidth minus actual flow data rate) to the actual flow data rate. The cumulated density function (CDF) of the normalized relative bandwidth is shown by Fig. 3.7. Negative values in the normalized relative bandwidth means that provisioning bandwidth is less than 


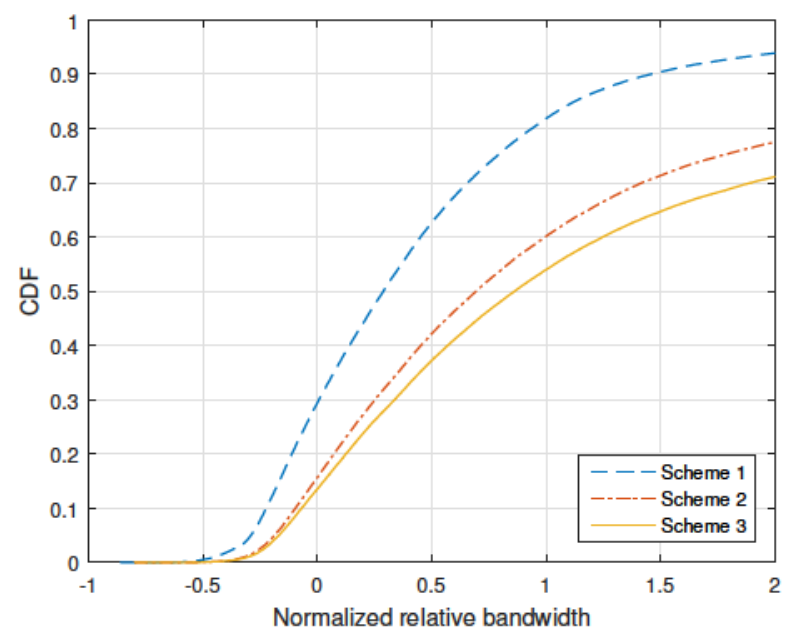

Figure 3.7: CDF of normalized provisioned bandwidth.

actual flows' requests. Obviously, by utilizing in-network caches and the robust optimization, our proposed schemes (Scheme 3) reduce the under-provisioning ratio from $30 \%$ to $15 \%$. Moreover, in-network caches and the robust optimization boost the data rate significantly by observing that the NRB at the point of $50 \%$ increases from 0.25 to 0.8 . Considering the worst $10 \%$ samples, our proposed scheme also improves them from -0.25 to -0.1 .

\subsection{Conclusions}

In this chapter, we jointly studied admission control and resource allocation for WNV. We proposed a two-stage VN embedding mechanism for enabling virtual network admission control and bandwidth provisioning. Firstly, we decoupled the admission control and physical resource leasing from physical resource allocation and bandwidth provisioning, as they are in different time scales. Then, we formulated the virtual network admission control and physical resource leasing as an robust optimization problem, which maximizes the utility of mobile virtual network provider. In addition, we took the uncertainty and fluctuation of flows rate into account and 
posed bandwidth provisioning as a set of chance constraints. Then, we approximated the admission control and bandwidth provisioning problems to convex problems by utilizing recent advances in robust optimization and the approximation technique. Simulation results were presented to show that the performance of physical resource reservation can be substantially improved and QoS requirements can be guaranteed. 


\section{Chapter 4}

\section{Enhancing QoE-aware Caching in SDWNs}

\subsection{Introduction}

Generally speaking, SDWNs can enable the reduction of complexity and cost of networks, equip programmability into wireless networks, accelerate evolution of networks, and even further catalyze fundamental changes in the mobile ecosystem $[28,29]$. The success of the SDWN will depend critically on our ability to jointly provision the backhaul and RANs [32]. Specifically, provisioning bandwidth of SDWNs includes forwarding decisions (cell selections and backhaul routing) and radio resource allocation [34]. [34] proposes a multi-path traffic engineering formulation for downlink transmission considering both backhaul and radio access constraints. Moreover, the link buffer status is used as feedback to assist the adjustment of flow allocation. Based on [34], the authors of [42] extend flow control of SDWNs to real-time video traffic specifically. This research proposes an online method to estimate the effective rate of video flows dynamically. Min flow rate maximization of SDWNs is investigated in [32] with jointly considering flow control and physical layer interference management problem by weighted-minimum mean square error algorithm. 
In-network caching that can efficiently reduce the duplicate content transmission [7], has shown that access delays, traffic loads, and network costs can be potentially reduced by caching contents in wireless networks $[2,12,13]$. To successfully combine caching and wireless networks, significant works (e.g., [43, 47, 51, 102, 103]) have been done concerning utilizing and placing contents in the caches of BSs. In the line of caching at the wireless network, the authors of $[43,45]$ formulate delay minimization problems by optimally caching contents at the SBSs. Meanwhile, [46,47,103] propose to utilize caching for enhancing MIMO cooperation without payload backhaul. Unfortunately, the research does not take the real-time wireless network and traffic status into account. In [51], dynamic caching is proposed to consider the mobility of users, but network resources, such as the backhaul capacity and the spectrum, are not discussed in this research.

As the QoE of streaming video services mainly includes video resolutions, buffering delays and the frozen (stalling) time [40] and [41], the SDWN (e.g., provision QoS [42]) and wireless edge caching (e.g., reduce delay [43]) appear as promising candidates to enhance QoE. However, to the best of our knowledge, QoE-aware joint optimization of the network and cache resources in SDWNs has been largely ignored in the existing research. Unfortunately, the combination of those issues are not straightforward, as several challenges are induced by this joint optimization observed as follows. First, bandwidth provisioning in SDWNs should be content-aware, which means it should assign network resources to users based on the caching status and the improvement of the QoE. Second, to enhance the hitting ratio of caches (utilization), caching strategies should be proactive according to the current traffic and resource status, behaviors of users, as well as the requirements of the QoE. Third, since video SDN flows from service providers usually have minimum requirements, the overall QoE performance of the network needs to be guaranteed. 
Thus, to address those issues, in this chapter, we propose to jointly optimize QoE of video streaming, bandwidth provisioning and caching strategies in SDWNs with limited network resources and QoE requirements. The distinctive technical features of this chapter are listed as follows:

- To decrease the content delivery latency and improve the utilization of the network resources and caches, we design a novel mechanism to jointly provide proactive caching, bandwidth provisioning and adaptive video streaming. BSs are requested to retrieve data in advance dynamically according to the behaviors of users, the current traffic and the resource status.

- To cope with the limited resources and the quality of service requirements, we formulate a novel optimization problem regarding the QoE-aware bandwidth provisioning in SDWNs with jointly considering in-network caching strategy.

- The caching problem is decoupled from the bandwidth provisioning problem by deploying the dual-decomposition method. Additionally, we relax the binary variables to real numbers so that those two problems are formulated as a linear problem and a convex problem, respectively, which can be solved efficiently.

- Algorithms are proposed to achieve the sub-optimum solution by solving the relaxed problem and utilizing a rounding up method that recovers relaxed variables to binary.

- A practical implementation of SDWNs is proposed, and the corresponding messages exchange of the control plan in SDWNs is designed.

The rest of this chapter is organized as follows. Section 4.2 introduces the system model and formulate the proposed problem. Section 4.3 presents two proposed algorithms and the corresponding analysis. Simulation results are discussed in Section 4.4. Finally, we conclude this study in Section 4.5. 


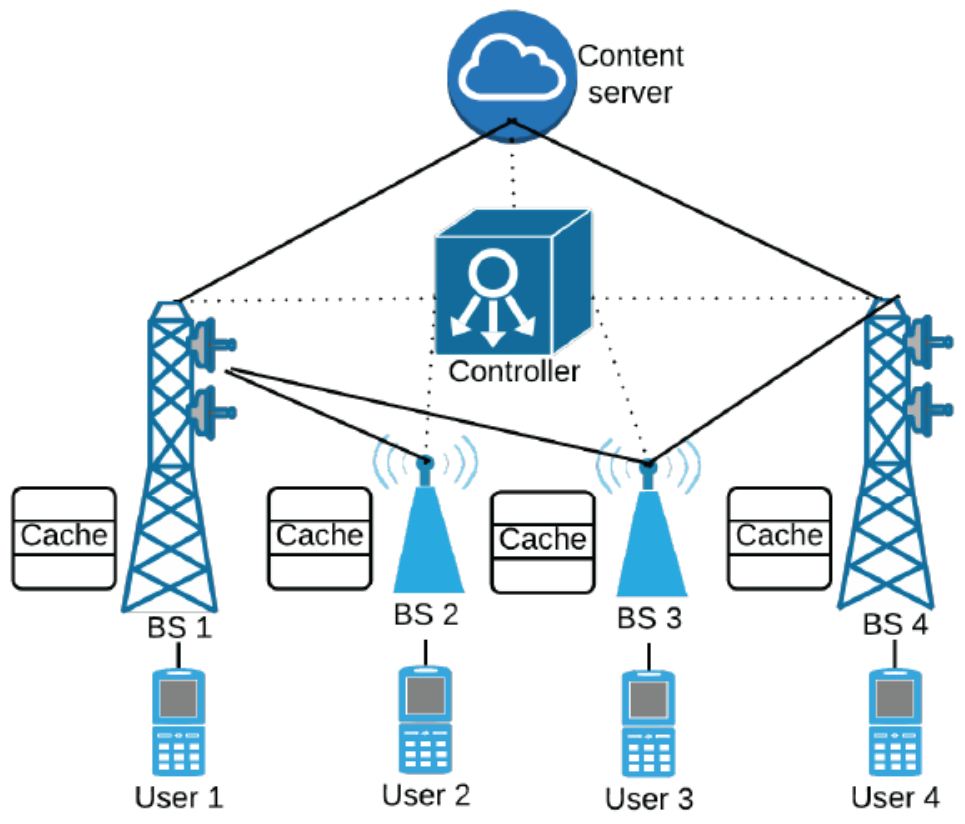

Figure 4.1: Network architecture of the cache-enabled SDWN.

\subsection{System Model and Problem Formulation}

In this section, we present the system model of the HetNet, dynamic caching, service QoE and related assumptions. The notations that will be used in the rest of this chapter are summarized in Table 4.1.

\subsubsection{Network Model}

\section{Wireless Communication Model}

In this chapter, we consider the downlink transmission case in a software-defined HetNet comprised of a set $\mathcal{J}$ of cache-enabled BSs, such as MBSs and SBSs. A central SDN controller is deployed to control the network including caching strategies and bandwidth provisioning. BSs connect to the $\mathrm{CN}$ through wired backhaul links with fixed capacities (e.g., bps). A content server is physically located at a core router 
Table 4.1: Notations of Chapter 4.

\begin{tabular}{|c|c|}
\hline Notation & Definition \\
\hline $\mathcal{J}$ & The set of mobile BS nodes (e.g., MBSs, SBSs) \\
\hline$J=|\mathcal{J}|$ & Number of BSs \\
\hline$j, l$ & BS, $j=0$ means content cloud library \\
\hline$a_{l j} \in\{0,1\}$ & Link status between the BS $j$ and the BS $l$ \\
\hline$R_{l j}^{\max }(\mathrm{bps})$ & Backhaul link capacity between the BS $j$ and the BS $l$ \\
\hline $\mathcal{I}_{j}, \mathcal{I}$ & The set of users \\
\hline $\mathcal{I}_{j}^{\pi}$ & The predicted (potential) set of users served by BS $j$ \\
\hline$i$ & Wireless user \\
\hline$g_{i j}$ & Large scale channel gain between $i$ and the BS $j$ \\
\hline$\sigma_{0}$ & Noise power spectrum density \\
\hline$p_{j}($ Watts / Hz) & Normalized transmission power of the BS $j$ \\
\hline$w_{i j}(\mathrm{~Hz})$ & Assigned spectrum bandwidth of the user $i$ from $j$ \\
\hline$W_{j}(\mathrm{~Hz})$ & Total spectrum bandwidth of $j$ \\
\hline$\gamma_{i j}$ & Received SINR of the user $i$ served by the BS $j$ \\
\hline$\Gamma_{i j}$ & Spectrum efficiency of the user $u$ served by the BS $j$ \\
\hline$C, C_{j}$ & The total number of contents and the storage capacity of the BS $j$ \\
\hline$h_{i j} \in\{0,1\}$ & Hitting event indicator between the user $i$ and the BS $j$ \\
\hline$c_{i j} \in\{0,1\}, \tilde{c}_{i j} \in[0,1]$ & Binary and relaxed dynamic caching indicator \\
\hline$\delta_{0}$ (seconds) & Evaluation time (scheduling cycle time) \\
\hline$\pi_{i j}$ & Predicted probability of the user $i$ served by the BS $j$ \\
\hline$Q$ & Number of total video resolution levels \\
\hline$q \in\{1, \ldots, 6\}$ & Video resolution level indicator \\
\hline$s_{q}$ & The MV-MOS of the resolution $q$ \\
\hline$v_{q}$ & Minimum required transmission data rate of the resolution $q$ \\
\hline$x_{q i} \in\{0,1\}, \tilde{x}_{q i} \in[0,1]$ & Binary and relaxed resolution indicator of the user $i$ \\
\hline$d_{j}$ & The average backhaul transmission delay of the BS $j$ \\
\hline$b_{0}$ (seconds) & Minimum buffer length to play video \\
\hline$b_{q i}$ (seconds) & Current buffer length of resolution $q$ at the user $u$ \\
\hline$S_{0}$ (seconds) & The MV-MOS requirements from service providers \\
\hline$U$ & Objectives, utilities \\
\hline$\iota$ & Consistence \\
\hline$r_{i j}^{c}(\mathrm{bps})$ & Required data transmission rate of caching \\
\hline$r_{l j}^{f}(\mathrm{bps}), r_{i j}^{a}(\mathrm{bps})$ & Provisioned bandwidth for backhaul links and air interfaces \\
\hline$\lambda, \mu, \nu$ & Dual variables \\
\hline$G, L_{j}$ & Dual functions \\
\hline$\Omega_{i j}$ & Caching margin gain \\
\hline$N_{j}$ & Number of cachable contents \\
\hline
\end{tabular}


(content delivery networks) in the $\mathrm{CN}$ or the source server. Moreover, as shown in Fig. 5.1, some BSs (e.g., SBSs) connect to the CN through MBSs. Without loss of generality, each BS may have multiple links to be connected in the networks (e.g., SBS 3 in Fig. 5.1). We use link indicator $a_{l j}$ to denote the link status between the BS $l$ and the BS $j . a_{l j}=1$ means the BS $l$ is the next hop of the BS $j$ with a fixed link capacity $R_{l j}^{\max }$ (bps); otherwise $a_{l j}=0$. Since it is the downlink case, $a_{l j}=1$ implies $a_{j l}=0$. Specially, we use $j=0$ to indicate the CN. Let $r_{l j}^{f} \in \mathbb{R}^{+}$denote the provisioning bandwidth of the BS $j$ for its next hop BS $l$, following constraints (backhaul limitation) need to hold

$$
r_{l j}^{f} \leq R_{l j}^{\max }, \forall j, l \in \mathcal{J}
$$

Let $\mathcal{I}_{j}$ denote the set of users served by BS $j$ and each user $i$ requests a video flow with certain data rate requirements. The set $\mathcal{I}:=\mathcal{I}_{1} \cup \ldots \cup \mathcal{I}_{j}$ of users means the total users set. To enforce single association, we let $\mathcal{I}_{1} \cap \mathcal{I}_{j}=\varnothing$ and any user is served by the BS that provides the best spectrum efficiency (SE). Association schemes are wellstudied in wireless networks from many aspects by effective research [81,104,105]. In this chapter, to simply our analysis, we do not consider any advanced interference management and power allocation schemes. We assume that the spectrum used by different users within one BS is orthogonal, which means there is no intra-cell interference between users associated with the same BS. The spectrum reuse factor for all cells is one (overlaid), which means inter-cell downlink interference are considered. A fixed equal power allocation mechanism is used, where the normalized transmit power on $\mathrm{BS} j$ is $p_{j}$ Watts/Hz regardless of positions and allocated spectral of users.

If $i \in \mathcal{I}_{j}$, the signal-to-interference plus noise (SINR) $\gamma_{i j}$ can be determined by

$$
\gamma_{i j}=\frac{g_{i j} p_{j}}{\sigma_{0}+\sum_{l, l \neq j} g_{i l} p_{l}}
$$


where $g_{i j}$ is the channel gain between the user $i$ and the BS $j, \sigma_{0}$ is the power spectrum density of additive white Gaussian noise and $\sum_{l, l \neq j} g_{i l} p_{l}$ is the aggregated received interference. As the small-scale fading varies much faster than the caching and the bandwidth provisioning, the channel gain only includes large-scale pathloss and shadowing. We deploy the same model used in [81] to calculate the pathloss and apply shadowing.

Accordingly, by using Shannon bound to get the spectrum efficiency (SE) $\Gamma_{i j}=$ $\log \left(1+\gamma_{i j}\right)$, the provisioning (achievable) radio access data rate $r_{i j}^{a} \in \mathbb{R}^{+}$for the user $i$ with the BS $j$ can be calculated as:

$$
r_{i j}^{a}=w_{i j} \log \left(1+\gamma_{i j}\right)
$$

where $w_{i j}(\mathrm{~Hz})$ is available spectrum bandwidth of the BS $j$ allocated to the user $i$. Since the spectrum bandwidth of each BS $j$ is limited by $W_{j}$, the following constraints have to hold

$$
\sum_{i \in \mathcal{I}_{j}} \frac{r_{i j}^{a}}{\Gamma_{i j}} \leq W_{j}, \forall j \in \mathcal{J}
$$

\section{Proactive Wireless Edge Caching Model}

To improve the caching performance, BSs can proactive cache contents that may be requested users. The probability that a cached content in the BS $j$ will be used by the user $i$ is assumed to be $\pi_{i j}$. In practice, $\pi_{i j}$ depends on the user mobility pattern [106] (current location, moving velocity and direction, and unpredictable factors) and the popularity of the content [2,51,103]. The research about behaviors of users is current understudying by both academic and industry [7,107]. It should be noted that the calculation of this probability is beyond the research of this dissertation and we assume that $\pi_{i j}$ is known in advanced by the SDWN controller (before each 
scheduling circle starts). To evaluate the effect of $\pi_{i j}$, we test our performance with two different $\pi_{i j}$ setups in the simulation. Let us use $\mathcal{I}_{j}^{\pi}$ to denote the potential set of users that may be probably served by the BS $j$, which can be formally defined as $\mathcal{I}_{j}^{\pi}:=\left\{i \mid \pi_{i j} \neq 0, i \in \mathcal{I}\right\}$.

In this proposed scheme, we assume the total number of content is $C$ stored at the content server (cloud centers or the Internet source) and each content has the normalized size of 1 . This assumption is reasonable because we can slice the video content into chunks with the same length. Our BSs are assumed to be cache-enabled, so there is a physical cache at each BS $n$ with capacity $C_{j}$. As any BS only has limited storage capability, $C_{j}$ is much smaller than cloud center $\left(C_{j} \ll C, \forall j\right)$ and the cache placement decision is made by the SDN controller. We assume that the SDN controller knows all the information about content retrieving requests from users and cache placement status of all BSs. Let us use a binary parameter $h_{i j}$ to denote a hitting event between the user $i$ and the BS $j . h_{i j}=1$ means the content requested by the user $i$ can be found at the BS $j$ and $h_{i j}=0$ means opposite. According to the control signaling by the SDN controller, contents are pulled actively by BSs through wired backhaul links, an example shown in Section 4.3.5.

Denote $c_{i j} \in\{0,1\}$ to be the binary decision variable used to control whether the content $i$ (potentially requested by user $i$ ) is going to be placed at BS $j$ or not. The bandwidth required to cache the content requested by $i$ is pre-defined as $r_{i}^{c}$ (bps) that is fixed. Hence, the provisioned bandwidth for caching in the BS $j$ is $\sum_{i \in \mathcal{I}_{j}^{\pi}} c_{i j} r_{i}^{c}$. Note duplicated caching is avoided as the SDN controller knows the cached contents of every BS. In other words, if the content has been cached in the BS $j$, it is unnecessary to cache again, namely $c_{i j}=0$ if $h_{i j}=1$. Since Each BS $j$ has limited cache space, constraints should hold to ensure caching strategy is limited in the empty space of 
the cache of each access BS $j$.

$$
\sum_{i \in \mathcal{I}_{j}^{\pi}} c_{i j} \leq C_{j}, \forall j \in \mathcal{J}
$$

\section{Video QoE Model}

In this chapter, we assume the $q$-th resolution of any video requires a data rate $v_{q}$ (bps) and U-vMOS $s_{q}$ (from 1 to 5) proposed in [38]. For example, [38] points out that $4 \mathrm{k}$ video average requires $25 \mathrm{Mbps}$ data rate and can be quantified to a U-vMOS 4.9 out of 5 . Practically, $v_{q}$ depends on video coding schemes and the video content, which are varying with time. Nevertheless, since the purpose of this dissertation is to maximize the wireless network performance dynamically instead of video services, we assume the required data rate is a fixed value $v_{q}$ over research time for all streams.

We define $x_{i q}$ as the resolution indicator of the user $i$. Specifically, if the $q$-th resolution of the video is selected by the user $i, x_{i q}=1$; otherwise, $x_{i q}=0$. Thus, for any user $i$, the experienced $\mathrm{U}$-vMOS is $\sum_{q} x_{i q} s_{q}$ and the required data rate is $\sum_{q} x_{i q} v_{q}$. Since users only can select one level of resolution at the same time, following constraints should hold:

$$
\sum_{q} x_{i q}=1, \forall i \in \mathcal{I}
$$

Moreover, to guarantee the video service QoE of the overall network, we should guarantee the overall average U-vMOS higher than acceptable value $S_{0}$, namely,

$$
\frac{1}{I} \sum_{i \in \mathcal{I}} \sum_{q} s_{q} x_{i q} \geq S_{0}
$$

Usually, the $S_{0}$ is provided by service providers or network operators to control the overall performance of a certain area. 
In [40] and [41], the authors point out that the stalling (video interruption) degrades users experience more severe even compared to initial latency because it disrupts the smoothness of streaming videos. It happens when the buffer $b_{i q}$ (bits) is exhausted, lower than a threshold $b_{0}$. In order to avoid this "annoy" factor, in this dissertation, we request the wireless network to maintain the buffer higher than a threshold $b_{0}$ (bits) at user devices buffer, so that the user can get smooth streaming video experience. This proactive buffer management that feedback the buffer status of users to the network has been proposed in the existing research [42, 52, 55]. To maintain $b_{0}$ of video, for any user $i$, it should follow constraints

$$
\delta \sum_{j \in \mathcal{J}} r_{i j}^{a}+\sum_{q} x_{i q} b_{i q}-\delta \sum_{q} x_{i q} v_{q} \geq b_{0}, \forall i \in \mathcal{I}
$$

where $\delta$ is a predefined time window unit in second. Every $\delta$ seconds, the system adaptively selects the resolution of the demanded video based on buffer status $b_{i q}$ and available data rate $\sum_{j \in \mathcal{J}} r_{i j}^{a}$. (4.8) means that the amount of downloaded data and buffered data must support to playback average $\delta(\mathrm{s})$ and maintain at least $b_{0}$ buffer.

\subsubsection{Problem Formulation}

In this subsection, an optimization problem maximizing the gains from wireless edge caching constrained by physical resource and service QoE is proposed.

The purpose of this study is to improve the overall wireless edge caching performance by considering caching strategies, network status and QoE provisioning, so it has to choose an appropriate network utility function $U$. Firstly, the caching utilization may lead various but correlated benefits (e.g., alleviated backhaul, delay reduction, network costs). Secondly, as this study is QoE-aware where the delay is one of the most important metrics, we chose delay reduction ${ }^{1} d_{j}$ (seconds) as the

\footnotetext{
${ }^{1}$ We use the average backhaul downlink latency as the measurement value.
} 
gain of caching at the wireless edge [43]. Thus, shown as following, we define a logarithm-based objective function as the utility function, which is equivalent to the proportional fairness scheme in a long term view [105]:

$$
U(\mathbf{c})=\sum_{j \in \mathcal{J}} \log \left(\sum_{i \in \mathcal{I}_{j}^{\pi}} \pi_{i j} \bar{h}_{i j} c_{i j} d_{j}+\iota\right)
$$

where $\bar{h}_{i j}=1-h_{i j}$ and $\iota \geq 1$ is consistences to guarantee $U(\mathbf{c})$ does not fall into negative infinity ${ }^{2}$. The product of $\bar{h}_{i j}$ and $c_{i j}$ enforces $c_{i j}$ to be different from $h_{i j}$ if $h_{i j}=1$, which is equivalent to the case that duplicated caching is avoided. The probability $\pi_{i j}$ can be interpreted as a weighted factor that represents the success of caching. To lighten the notations, we let $H_{i j}=\pi_{i j} \bar{h}_{i j} d_{j}$. We select a logarithm-based objective function due to following features that are perceived [105]:

- Component-wise monotonic increase brings that larger caching gain yields larger utility;

- Convexity can guarantee the convergence and efficiency of the algorithm;

- Fairness-awareness gives a desired balance between the overall network and the single user.

To utilize the physical resource efficiently, the total provisioned bandwidth of flows going out the BS $j$ should be less than the total bandwidth of flows coming into the corresponding BS. Otherwise, allocated spectrum or backhaul links will be left

\footnotetext{
${ }^{2}$ When either $\mathcal{I}_{j}^{\pi}=\varnothing$ or $\pi_{i j} \bar{h}_{i j}=0, \forall i \in \mathcal{I}_{j}^{\pi}$, the objective function may result in an infeasible problem.
} 
unused. The flow conservation constraint for the BS $j$ can be written as

$$
\underbrace{\overbrace{\sum_{l \in \mathcal{J}} a_{l j} r_{l j}^{f}}^{\text {forwarding }}+\overbrace{\sum_{i \in \mathcal{I}_{j}} \bar{h}_{i j} r_{i j}^{a}}^{\text {radio }}+\overbrace{\sum_{i \in \mathcal{I}_{j}^{\pi}} c_{i j} r_{i}^{c}}^{\text {caching }}}_{\text {out flows }} \leq \underbrace{\sum_{l \in \mathcal{J} \cup 0} a_{j l} r_{j l}^{f}}_{\text {in flows }}, \quad \forall j \in \mathcal{J} .
$$

The first term of left (4.10) is all reserved bandwidth for flows forwarded to next hop BSs. The second term is the summation of all provisioned bandwidth for bearing users' radio access flows. Obviously, if $\bar{h}_{i j}=0$ that means we can find existing data (cached in previously caching circles) on the BS $j$ for the user $i$ so that the backhaul consumption is avoided. The third term is all caching flows.

Thus, given the objective function, and constraints, we can define the joint flow bandwidth provisioning and wireless edge cache placement problem P0 as follows:

$$
\begin{aligned}
& \text { P0 : } \max _{\substack{\mathbf{r}^{a}, \mathbf{r}^{f} \in \mathbb{R}^{+} \\
\mathbf{c}, \mathbf{x} \in\{0,1\}}} U \\
& \text { s.t. }(4.1),(4.4),(4.5),(4.6),(4.7),(4.8),(4.10)
\end{aligned}
$$

Unfortunately, problem (4.11), however, is difficult to be solved and implemented based on the following observations:

- The mix integer variables result in the problem a mix-integer non-linear problem (MINLP) that generally is NP-hard and intractable in scheduling [108];

- The complexity of solving (4.11) by using greedy or genetic methods will increase significantly with the increase of the number of users and (or) BSs;

- Cache indicators and flows bandwidth are decided by different layers and perform in different time scales. 


\subsection{QoE-aware Joint Bandwidth Provisioning and Caching}

In this section, an algorithm is proposed to solve the problem P0 in (4.11). As those integer variables block us to find a traceable algorithm, we firstly relax binary variables $c_{i j}$ and $x_{i q}$ to real numbers variables $\tilde{c}_{i j}$ and $\tilde{x}_{i q}$ bounded by $[0,1]$ so that the domain of variables is a convex set. This relaxation is a common way to deal with binary variables in wireless networks $[61,81,104,105]$. From a long term view, we can interpret this kind of relaxations as a partial allocation or average time sharing

portion. For example, a partial caching indicator $\tilde{c}_{i j}$ or resolution $\tilde{x}_{i q}$ means the portion of time when the BS $j$ indicate cache to the user $i$ or when the users $i$ selects the resolution level $q$, respectively. Moreover, by solving the relaxing binary variables, the upper bound of the proposed can be achieved. We will evaluation the gap between this upper bound and the final solution in our simulation.

After relaxing binary variables, it is clear that the feasible set of the revised problem (4.11) is a convex set because all constraints are linear constraints and variables domain is a convex set. Since the log-based objective function is a strict concave function regarding to $\tilde{c}_{i j}$, the problem (4.11) is transfered to a convex problem $\tilde{\text { P0 }}$ that can be solved effectively without effort by using general solvers. In the remaining subsections, we will present an algorithm based on dual-decomposition to solve the relaxed problem $\tilde{\mathbf{P 0}}$ and recover relaxed variables back to binaries.

\subsubsection{Proposed Caching Decoupling via Dual Decomposition}

This study aims to adaptively replace content in caches of BSs while optimize the bandwidth provisioning and the video resolution selection. Observed from the problem P0, we can consider that we use the spare backhaul bandwidth to cache content 
to BSs before users actually served (or potentially continued being served) with those BSs (or actually request the content) and select a best video resolution for each user based on allocated achievable data rate. Moreover, caches and bandwidth are actually belonging to different layers of the network. Usually, flow bandwidth are mostly restricted by physical resources (provisioned by media access control layer) and $x_{i q}$ depends on the achievable data rate. Differently, in-network cache is placed at the network layer or even higher layer. In addition, scheduling of the caching can be acting in a longer time period compared to flow scheduling. Fortunately, constraints coupling cache and bandwidth are only (4.10).

Thus, those features of P0 motivate us to adopt dual-decomposition method so that the caching problem can be separated from the bandwidth provisioning and the resolution selection. We form the partial Lagrangian function for the problem $\tilde{\mathbf{P} 0}$ by introducing the dual variables (backhaul prices) $\left\{\lambda_{j}\right\}$ for constraints (4.10). Then, the partial Lagrangian function can be shown as:

$$
\begin{aligned}
& G(\lambda)=\sum_{j \in \mathcal{J}} \log \left(\sum_{i \in \mathcal{I}_{j}^{\pi}} H_{i j} \tilde{c}_{i j}+\iota\right) \\
& -\sum_{j \in \mathcal{J}} \lambda_{j} \sum_{i \in \mathcal{I}_{j}^{\pi}} \tilde{c}_{i j} r_{i}^{c}-\sum_{j \in \mathcal{J}} \lambda_{j}\left(\sum_{l \in \mathcal{J}} a_{l j} r_{l j}^{f}+\sum_{i \in \mathcal{I}_{j}} \bar{h}_{i j} r_{i j}^{a}-\sum_{l \in \mathcal{J} \cup 0} a_{j l} r_{j l}^{f}\right)
\end{aligned}
$$

The dual problem (DP) is thus:

$$
\mathrm{DP}: \min _{\lambda \in \mathbb{R}^{+}} G(\lambda)=f_{c}(\lambda)+f_{r, x}(\lambda)
$$

where

$$
f_{c}(\lambda)=\underset{\mathbf{c} \in[0,1]}{\arg \max } \sum_{j \in \mathcal{J}} \log \left(\sum_{i \in \mathcal{I}_{j}^{\pi}} H_{i j} \tilde{c}_{i j}+\iota\right)-\sum_{j \in \mathcal{J}} \lambda_{j} \sum_{i \in \mathcal{I}_{j}^{\pi}} \tilde{c}_{i j} r_{i}^{c}, \quad \text { s.t. }
$$


and

$$
f_{r, x}(\lambda)=\underset{\substack{\mathbf{r}^{a}, \mathbf{r} f \in \mathbb{R}^{+} \\ \mathbf{x} \in[0,1]}}{\arg \max }-\sum_{j \in \mathcal{J}} \lambda_{j}\left(\sum_{l \in \mathcal{J}} a_{l j} r_{l j}^{f}+\sum_{i \in \mathcal{I}_{j}} \bar{h}_{i j} r_{i j}^{a}-\sum_{l \in \mathcal{J} \cup 0} a_{j l} r_{j l}^{f}\right),
$$

s.t. $(4.1),(4.4),(4.6),(4.7),(4.8)$

Let us introduce a parameter $z_{n}$ called extra bandwidth for each BS $j$ shown as:

$$
z_{j}=\sum_{l \in \mathcal{J} \cup 0} a_{j l} r_{j l}^{f}-\sum_{i \in \mathcal{I}_{j}^{\pi}} \tilde{c}_{i j} r_{i}^{c}-\sum_{l \in \mathcal{J}} a_{l j} r_{l j}^{f}-\sum_{i \in \mathcal{I}_{j}} \bar{h}_{i j} r_{i j}^{a}
$$

According to dual decomposition [109], a subgradient of $G(\lambda)$ is $z=\left[z_{1}, \ldots, z_{j}\right]^{T}$, we thus can update $\lambda$ based on:

$$
\lambda^{[k+1]}=\lambda^{[k]}-\alpha_{\lambda}^{[k]} z^{[k]},
$$

where $\alpha_{\lambda}^{[k]}$ is the step length at the iteration step $[k]$.

\subsubsection{Upper Bound Approach to Solving (4.14)}

In this part, a low complexity algorithm is proposed to solve the problem (4.14) so that the upper bound can be achieved. Firstly, to make our expression more compact, we define $y_{i j}=\lambda_{j} r_{i}^{c}$ that can be interpreted as the backhaul bandwidth cost of caching. Observe that $f_{c}(\lambda)$ can be further decoupled to each BS $j$, thus we focus on solving (4.14) for one BS. Let $\mu_{j} \geq 0, \forall j \in \mathcal{J}$ and $\nu_{i j} \geq 0, \forall j \in \mathcal{J}, \forall i \in \mathcal{I}_{j}^{\pi}$ be the dual variables associated with constraints (4.5) and $\tilde{c}_{i j} \leq 1$ of the problem given in (4.14), 
respectively. Then, the Lagrangian of (4.14) can be expressed as

$$
\begin{aligned}
L_{j}(\tilde{\mathbf{c}}, \mu, \nu) & =\log \left(\sum_{i \in \mathcal{I}_{j}^{\pi}} H_{i j} \tilde{c}_{i j}+\iota\right)-\sum_{i \in \mathcal{I}_{j}^{\pi}} y_{i j} \tilde{c}_{i j}-\mu_{j}\left(\sum_{i \in \mathcal{I}_{j}^{\pi}} \tilde{c}_{i j}-S_{j}\right)-\sum_{i \in \mathcal{I}_{j}^{\pi}} \nu_{i j}\left(\tilde{c}_{i j}-1\right) \\
& =\log \left(\sum_{i \in \mathcal{I}_{j}^{\pi}} H_{i j} \tilde{c}_{i j}+\iota\right)-\sum_{i \in \mathcal{I}_{j}^{\pi}}\left(y_{i j}+\mu_{j}+\nu_{i j}\right) \tilde{c}_{i j}+\mu_{j} S_{j}+\sum_{i \in \mathcal{I}_{j}^{\pi}} \nu_{i j}
\end{aligned}
$$

It is noted that $L_{j}(\tilde{\mathbf{c}}, \mu, \nu)$ is a continuous and differentiable function of $\tilde{c}_{i j}, \mu_{j}$, and $\nu_{i j}$. By differentiating $L_{j}(\tilde{\mathbf{c}}, \mu, \nu)$ with respective to $\tilde{c}_{i j}$, we can have the KarushKuhn-Tucker (KKT) conditions as

$$
\begin{aligned}
& \frac{\partial L_{j}}{\partial \tilde{c}_{i j}}=\frac{H_{i j}}{\left(\sum_{i \in \mathcal{I}_{j}^{\pi}} H_{i j} \tilde{c}_{i j}+\iota\right)}-\left(y_{i j}+\mu_{j}+\nu_{i j}\right) \leq 0, \forall i \in \mathcal{I}_{j}^{\pi} \\
& \tilde{c}_{i j}\left[\frac{H_{i j}}{\left(\sum_{i \in \mathcal{I}_{j}^{\pi}} H_{i j} \tilde{c}_{i j}+\iota\right)}-\left(y_{i j}+\mu_{j}+\nu_{i j}\right)\right]=0, \forall i \in \mathcal{I}_{j}^{\pi} \\
& \mu_{j}\left(\sum_{i \in \mathcal{I}_{j}^{\pi}} \tilde{c}_{i j}-S_{j}\right)=0, \forall i \in \mathcal{I}_{j}^{\pi} \\
& \nu_{i j}\left(\tilde{c}_{i j}-1\right)=0, \forall i \in \mathcal{I}_{j}^{\pi}
\end{aligned}
$$


From (4.19) and (4.20), we obtain an optimal cache allocation for fixed Lagrange multipliers as

$$
\tilde{c}_{i j}=\left[\frac{1}{\left(y_{i j}+\mu_{j}+\nu_{i j}\right)}-\frac{\iota+\sum_{i^{\prime} \neq i} H_{i^{\prime} j} \tilde{c}_{i^{\prime} j}}{H_{i j}}\right]^{+},
$$

where $[x]^{+}=\max \{x, 0\}$. As $H_{i j}=0$ leads $\left[\frac{1}{\left(y_{i j}+\mu_{j}+\nu_{i j}\right)}-\frac{\iota+\sum_{i^{\prime} \neq i} H_{i^{\prime} j} \tilde{c}_{i^{\prime} j}}{H_{i j}}\right] \rightarrow-\infty$, $\tilde{c}_{i j}=0$. In other words, since $H_{i j}=0$, any positive values of $\tilde{c}_{i j}$ do not increase the utility but only waste physical resource instead. To obtain (4.23), using a gradientbased search, the updated $\mu_{j}$ value is given by

$$
\mu_{j}^{[t+1]}=\left[\mu_{j}^{[t]}-\alpha_{\mu}^{[t]}\left(\sum_{i \in \mathcal{I}_{j}^{\pi}} \tilde{c}_{i j}-S_{j}\right)\right]^{+},
$$

where $[t]$ is the iteration index and $\alpha_{\mu}^{[t]}$ are sufficiently small step sizes. Similar to $\nu_{i j}$,

$$
\nu_{i j}^{[t+1]}=\left[\nu_{i j}^{[t]}-\alpha_{\nu}^{[t]}\left(\tilde{c}_{i j}-1\right)\right]^{+}
$$

where $\alpha_{\nu}^{[t]}$ are sufficiently small step sizes. We summarize the procedure used to get the optimal solution of the problem (4.14) in Table 4.2.

The problem (4.15) obviously is a linear problem that can be solved effortlessly by general methods (e.g. interior point method) that are polynomial time algorithms in the worst case. Moreover, since the problem (4.15) is similar to the problem (4.14), even simpler because of the linear objective function, we can use the same idea to solve the problem (4.15). 
Table 4.2: Algorithm for the wireless edge caching

\section{Upper bound algorithm of the wireless edge caching}

1: Initialize:

2: Set prescribed accuracy $\zeta$ and maximum number of iteration steps $T$

3: Set initial dual variables $\mu_{j} \leftarrow \mu_{j}^{[0]}, \forall j ; \quad \nu_{i j} \leftarrow \nu_{i j}^{[0]}, \forall i, j$

4: $t=1$

5: for $\zeta$ is not reached \&\& $t \leq T$ do

6: $\quad$ Update $\tilde{c}_{i j}^{[t+1]}$ according to (4.23)

7: $\quad$ Update $\mu_{j}^{[t+1]}$ according to according to (4.24)

8: $\quad$ Update $\nu_{i j}^{[t+1]}$ according to according to (4.25)

9: $\quad t=t+1$

10: end for

11: Output the optimal resource allocation policy

\subsubsection{Rounding Methods Based on Marginal Benefits}

Recall that we have relaxed the cache placement indicators $c_{i j}$ and video resolution $x_{i q}$ to real values between zero and one instead of binary variables. Thus, we have to recover them to binary values after we get the relaxed solution. The basic idea of the rounding method is that we select the 'best' users to utilize the caching resource and the 'highest' resolution for users under current resource allocation solution. In this chapter, $c_{i j}$ is recovered to binary based on the corresponding marginal benefit $[101,104]$.

Firstly, we calculate the marginal benefits of each user as

$$
\Omega_{i j}^{c}=\partial U / \partial \tilde{c}_{i j}=\frac{H_{i j}}{\left(\sum_{i \in \mathcal{I}_{j}^{\pi}} H_{i j} \tilde{c}_{i j}+\iota\right)} .
$$


Then, assuming $\tilde{c}_{i j} \in[0,1]$ is the achieved optimum solution, we can obviously calculate the available number of cache that can be updated at the BS $j$ as

$$
N_{j}=\left\lfloor\sum_{i \in \mathcal{I}_{j}^{\pi}} \tilde{c}_{i j}\right\rfloor .
$$

where $\lfloor x\rfloor$ means taking the maximum integer value that is less than $x$. In other words, $N_{j}$ means the maximum number of content segments that can be cached at the BS $j$ during the scheduling period. $N_{j}$ users can be selected from from $\mathcal{I}_{j}^{\pi}$, whose $O m e g a_{i j}^{c}$ are lager than other users. Formally, we can separate all users of BS $j$ to two sets $\mathcal{I}_{j}^{[\pi,+]}$ containing $N_{j}=$ elements and $\mathcal{I}_{j}^{[\pi,-]}$ containing the remaining users. $\mathcal{I}_{j}^{[\pi,+]}$ and $\mathcal{I}_{j}^{[\pi,-]}$ have following relationship:

$$
\max _{i \in \mathcal{I}_{j}^{[\pi,-]}} \Omega_{i j}^{c} \leq \min _{i \in \mathcal{I}_{j}^{[\pi,+]}} \Omega_{i j}^{c}
$$

Then, the caching indicator $c_{i j}$ can be recovered by

$$
c_{i j}=\left\{\begin{array}{cc}
1 & \text { if } i \in \mathcal{I}_{j}^{[\pi,+]} \\
0 & \text { otherwise }
\end{array}\right.
$$

The recovering of $x_{i q}$ is easier due to constraints (4.6). By assuming $r_{i j}^{a}$ is the achieved optimum solution, the method uses following rule:

$$
x_{i q}= \begin{cases}1, & \text { if } q=\underset{q}{\arg \max } s_{q}^{v}, \text { s.t. } v_{q} \leq \sum_{i \in \mathcal{J}} r_{i j}^{a} \\ 0, & \text { otherwise. }\end{cases}
$$

(4.30) is used to find the highest resolution whose required data rate is lower than the provided data rate. 
Table 4.3: Algorithm for the proposed joint allocation

The proposed joint allocation algorithm

1: Initialize:

2: Set prescribed accuracy $\epsilon$ and maximum number of iteration steps $K$

3: Set initial bandwidth prices $\lambda_{n} \leftarrow \lambda_{n}^{[0]}, \forall n$

4: $k=1$

5: for $\epsilon$ is not reached and $k \leq K$ do

6: $\quad$ Bandwidth provisioning and video resolution selection

7: $\quad$ Update $r_{l j}^{f}, r_{i j}^{a}$ and $\left\{\tilde{x}_{i q}\right\}$ by solving the problem (4.15)

8: $\quad$ Rounding $\left\{\tilde{x}_{i q}\right\}$ up to $\left\{x_{i q}\right\}$ according to (4.30)

9: $\quad$ Update $\tilde{c}_{i j}$ by solving the problem (4.14)

10: Calculate the marginal benefits and available cache according to (4.26) and (4.27)

11: $\quad$ Form $\mathcal{I}_{j}^{[\pi,-]}$ and $\mathcal{I}_{j}^{[\pi,+]}$ based on selecting users according to (4.28)

12: $\quad$ Rounding $\left\{\tilde{c}_{i j}\right\}$ up to $\left\{c_{i j}\right\}$ according to (4.29)

13: Update $\lambda^{[k+1]}$ according to (4.17)

14: $\quad k=k+1$

15: end for

16: Output the optimal resource allocation policy

As the two relaxed subproblems have been solved and variables are rounded up to binaries, the solution of the original problem $\mathbf{P 0}$ is obtained. A complete description of the overall proposed scheme is stated in Table 4.3.

\subsubsection{Computational Complexity, Convergence and Optimal- ity}

As we mentioned above, the problems formulated in (4.11) is nonlinear integer optimization problems and is NP-hard without computational efficient algorithms to obtain the optimal solution [108]. Exhaustive searching method can be used to find a solution. However, for a dynamic caching SDWN system with $I$ users and $J$ BSs, the computational complexity of exhaustive searching method is about $\mathcal{O}\left((J+1)^{I}\right)$ even 
we ignore the calculation of spectrum and backhaul allocation, which is tremendously high and unacceptable for practical implementation.

Alg. 4.2 is a polynomial time algorithm with the computational complexity of $\mathcal{O}(I J)$. The computational complexity of solving the problem (4.15) is $\mathcal{O}(\max \{I, J\} J)$ leading it as a polynomial time algorithm as well. The computational complexity of the proposed dual-decomposition Alg. 4.3 is $\mathcal{O}(\max \{I, J\} J)$. The detailed analysis of computational complexity can be found in Appendix B. Obviously, our proposed scheme reduce the computational complexity significantly, compared to exhaustive searching method.

Since either the inner loop in Alg. 4.2 or the outer loop in the proposed Alg. 4.3 is used to solve a convex problem which is proved to converge to the exact marginal. However, the precise conditions and the initial bandwidth prices used in dual-decomposition are not able to be decided before a practical implementation. The optimality cannot be guaranteed since we are solving an NP-hard problem. However, as we can get the upper bound of the proposed problem, we can use it as a replacement for the optimal solution to evaluate our proposed algorithm empirically. Thus, simulation results are used to test the convergence and the optimality of the proposed algorithm Fig. 4.4 in Section 4.4.

\subsubsection{Implementation Design in SDWNs}

To illustrate the utilization of Alg. 4.3 into SDWNs, we give an instance presented by a diagram and a signaling message exchange example in Fig. 4.2 and Fig. 4.3.

Firstly, as shown in Fig. 4.2, in the control plane, the SDN controller updates the network status according to feedbacks from BSs, then predict the users' behaviors to calculate $\left\{\pi_{i j}\right\}$. By using this information, the SDN controller calls our proposed Alg. 4.3 to calculate the provisioned bandwidth and caching strategy for each flow $i$ and 


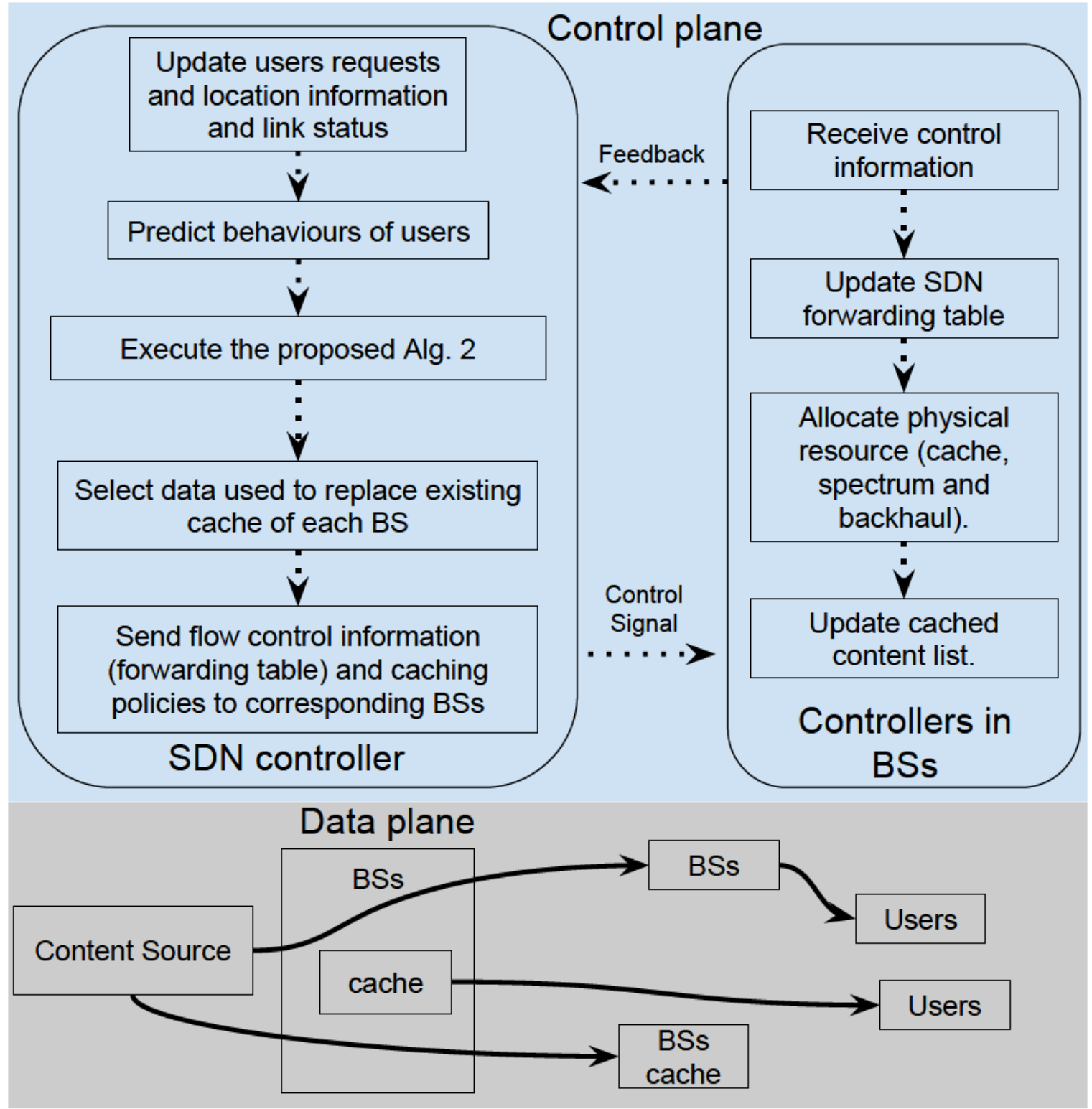

Figure 4.2: The flow diagram in the proposed cache-enabled flow control in SDWNs.

BS $j$. After this, the SDN controller sends the control information to selected BSs and content source (the cloud center or the public networks). According to the control information, BSs and other network elements (e.g., potential routers) will update their SDN forwarding tables and perform resource allocation (e.g., cell association and scheduling). In the data plane, flows carrying contents requested by users can 


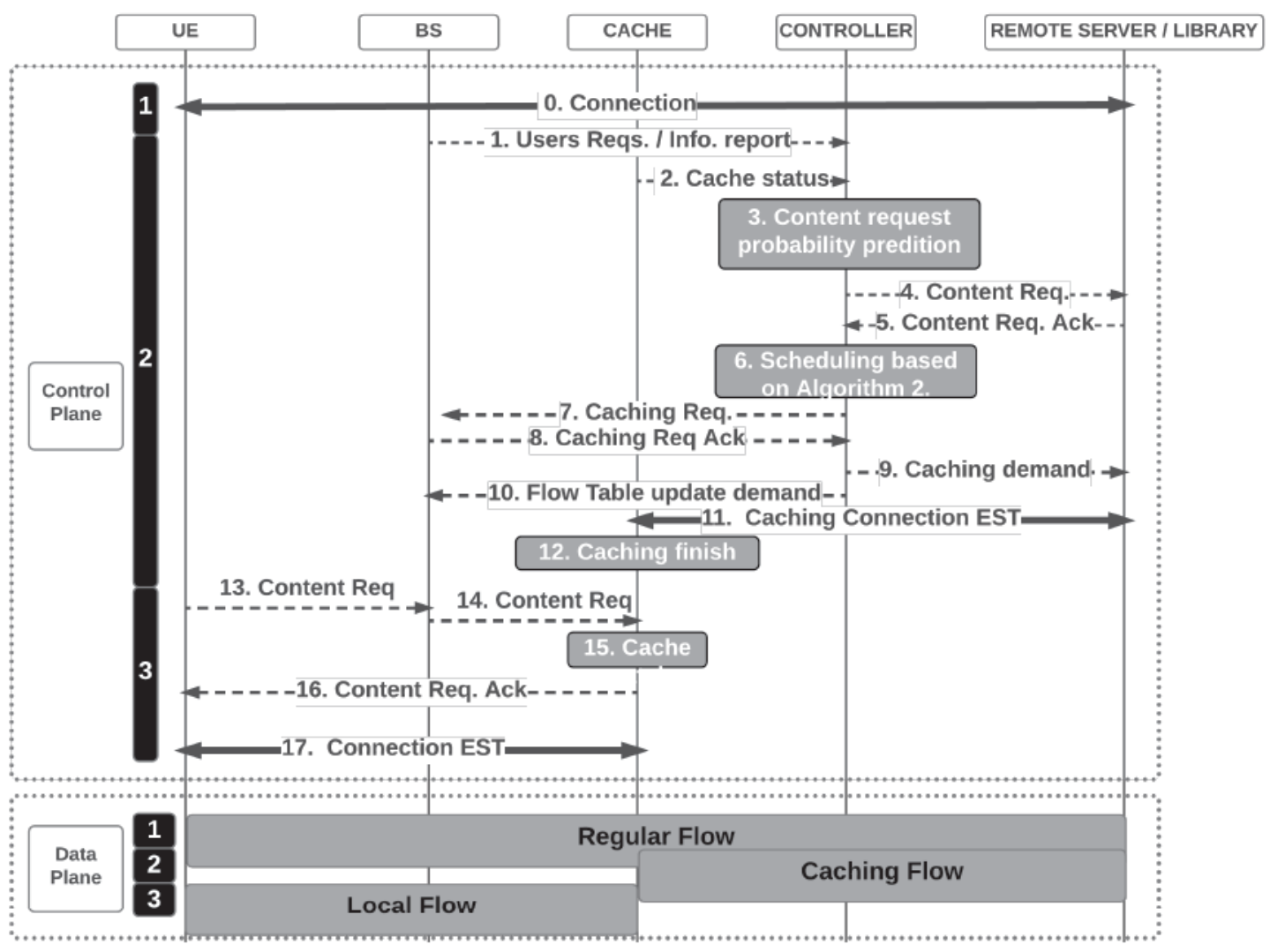

Figure 4.3: Signaling exchange in cache-enabled SDWNs.

pass networks elements selected by the control plane and to users.

A signaling message exchange presented by Fig. 4.3 is designed to realize the proposed scheme in SDWNs. There are three phases in this message exchange implementation, which are regular connection, caching preparation and caching delivery denoted by phase 1 , phase 2 , and phase 3 . In phase 1 , user equipments (UEs) have the regular connection with remote servers to download the video as shown by step 0 in the control plane. Accordingly, at the data plane, the data flows go to UEs directly from the servers.

In phase 2 , from steps 1 to 2 , the BS deliver the user information to the controller, then the prediction of the content request probability $\pi_{i j}$ can be conducted by the 
controller in step 3. Based on those, the controller can request the content from servers shown by steps 4-5. It should be noted, in these two steps, only content information is retrieved and used for scheduling. Meanwhile, the controller runs our proposed Alg. 4.3 , and afterward gets the scheduling decisions in terms of association, flow routes and caching strategies. By getting the caching allocation, caching request (step 7) will be sent to the corresponding BS and the following acknowledge signaling (steps 8-9) will be replied to the controller if caches at those the BS are available. Step 10 is used to send caching demands to the servers and flow table update demands are sent by step 11 to the BS. If all done, the caching connection is established between the BS and the server. In the corresponding data plane, caching flows transmit data from servers to the BS. It should be noted that phase 2 and phase 1 may be overlapped because the caching and the streaming can happen at the same time.

In phase 3, the user send the content request to the BS (steps 13-15). If the content is matched in the caches of associated the BS, the cache will send the acknowledge message back to the UE to establish the connection (from steps 16-17). After establishing the connection, the relevant data flows become local flows.

\subsection{Simulation Results and Discussions}

Simulation results are presented in this section to demonstrate the performance of the proposed scheme. The number of available videos is 100000 , with popularity following a Zipf distribution with exponent 0.56, following [43]. In the simulation, we consider a cellular network including 57 BSs that cover a $400 \mathrm{~m}$-by-400m. 9 BSs are core BSs (macro cells) that connect to the core network and the SDN controller directly. The remaining $48 \mathrm{BSs}$ (small cells) connect to the core networks through these 9 BSs. Besides those, perfect synchronization is assumed in this chapter. SISO case is considered in this chapter, but it is attractive to extend it to MIMO in the 
future work. The remaining simulation parameters are summarized in Table 4.4. The values with $*$ mean default values. The information on video streams, such as required data rate and corresponding MV-MOS, are shown in Table 4.5.

In our simulations, we compare five different schemes: the no-caching scheme (baseline (no cache)) that delivers video traffic in wireless networks without caching; the static caching (baseline) scheme (caches is fixed in the research period) with considering resource allocation, similar to what is discussed in [43,47], and the proposed dynamic caching scheme that replaces the caches with considering resource allocation and video quality selection. Two scenarios of the content request probability $\pi$ are tested. One is high probability ranging at $(0.5-0.95]$ and the other is low probability ranging at $(0-0.5]$. The high probability means that the user behavior is easier to predict (e.g., watching popular video and tractable paths) and the low probability means the user may quickly change the watched video and wander in the city.

\subsubsection{Algorithm Performance}

Fig. 4.4 demonstrates the evolution of the proposed dual decomposition algorithm for different initial values of $\lambda_{j}$. The iteration step $k$ refers to the main loop iteration of Algorithm 4.3. At each step we calculate the differences between obtained utility $U^{[k]}$ and the optimum utility $U^{*}$ by solving the relaxed problem (upper bound) $\tilde{\mathbf{P 0}}$. In most simulation instances shown in Fig. 4.4, we see that the proposed algorithm converges to a fixed point. It can be observed that the iterative algorithm converges to the optimal value within ten steps when $\lambda=0.05$, which means the optimum dynamic caching strategy and the bandwidth provisioning can be achieved within a few iterations. However, Fig. 4.4 also suggests that some inappropriate initial values of $\lambda$ may result in worst convergence speed. As shown in Fig. 4.4, its performance can approach the upper bound with a slight gap, especially after ten steps, which 
Table 4.4: Simulation parameters

\begin{tabular}{l|l}
\hline Network parameters & value \\
\hline \hline geographic area & $400 \mathrm{~m}-$ by- $400 \mathrm{~m}$ \\
number of BSs & 9 macro cells, 48 small cells \\
number of users & $30-120,80^{*}$ \\
frequency bandwidth $(\mathrm{MHz})$ & 20 \\
frequency reuse factor & 1 \\
transmission power profile & SISO with maximum power; $49 \mathrm{dBm}($ macro), $20 \mathrm{dBm}$ (small) \\
& pathloss:L(distance) $=34+40 \log ($ distance); \\
propagation profile [81] & lognormal shadowing: $8 \mathrm{~dB} ;$ no fast fading \\
power density of the noise & $-174 \mathrm{dBm} / \mathrm{Hz}$ \\
spectrum efficiency calculation & Shannon bound \\
backhaul capacity (Mbps) & macro to CN:500; small to macro: $25-160,40^{*}$ \\
average trasmission latency (ms) & RAN: $50 ;$ backhaul: 60 \\
prediction probability range & low:0-0.5; High:0.5-0.99 \\
total content & $10^{5}$ \\
content popularity profile & Zipf distribution with exponent 0.56 [43] \\
cache size at BSs & $300-1200,600^{*}$ \\
\hline
\end{tabular}

embraces a relatively acceptable solution.

\subsubsection{Network Performance}

\section{Delay}

Transmission delay is the key performance metric for video streaming in cache-enabled SDWNs. Users may give up watching the video because of the long waiting time for video buffering. In this subsection, we evaluate the average transmission delay performance and the results are shown in Figs. 4.5 and 4.6.

Fig. 4.5 shows the cumulative distribution function $(\mathrm{CDF})$ of round trip delay of users with different caching schemes and prediction accuracy respectively. The CDFs for dynamic and static caching all improve significantly at delay reduction 
Table 4.5: U-vMOS of video resolutions.

\begin{tabular}{r|l|l|l}
\hline$q$ & resolution & Required data rate $v_{q}$ & resolution vMOS $s_{q}$ \\
\hline \hline 6 & $4 \mathrm{k}$ or more & $25 \mathrm{Mbps}$ & 4.9 \\
5 & $2 \mathrm{k}$ & $12 \mathrm{Mbps}$ & 4.8 \\
4 & $1080 \mathrm{p}$ & $10 \mathrm{Mbps}$ & 4.5 \\
3 & $720 \mathrm{p}$ & $5 \mathrm{Mbps}$ & 4 \\
2 & $480 \mathrm{p}$ & $2 \mathrm{Mbps}$ & 3.6 \\
1 & $360 \mathrm{p}$ & $1.5 \mathrm{Mbps}$ & 2.8 \\
\hline
\end{tabular}

compared to no in-network caching case, showing a maximum $2.5 \mathrm{x}$ gain, in both low and high probability settings. Specifically, the proposed proactive caching boosts the performance of network delay deduction the most when we can keep caching contents with high probability to be requested. Almost 50\% users experience delay less than $60 \mathrm{~ms}$ because their backhaul latency is eliminated by the cache. Moreover, we can see even with low probability setting, the performance surpasses the static caching strategy, which implies that the proposed caching scheme should be deployed no matter the probability. It should be noted that the flat curves appeared in CDFs (between $60 \mathrm{~ms}$ to $80 \mathrm{~ms}$ ) due to the elimination of backhaul latency brought by deploying in-network caching.

Results shown in Fig. 4.6 compare the average delay performance among different caching schemes and prediction accuracies. In Fig. 4.6a and Fig. 4.6b, note that the average delay of users can be further reduced by increasing available physical resource such as available cache space or (and) backhaul bandwidth of each BS because more data are going to be cached when we have more resources. It is observed that the proposed dynamic caching scheme with high probability always achieves the lowest while the proposed dynamic caching scheme with low probability is also better than static caching scheme. However, when the probability is not high, increasing backhaul 


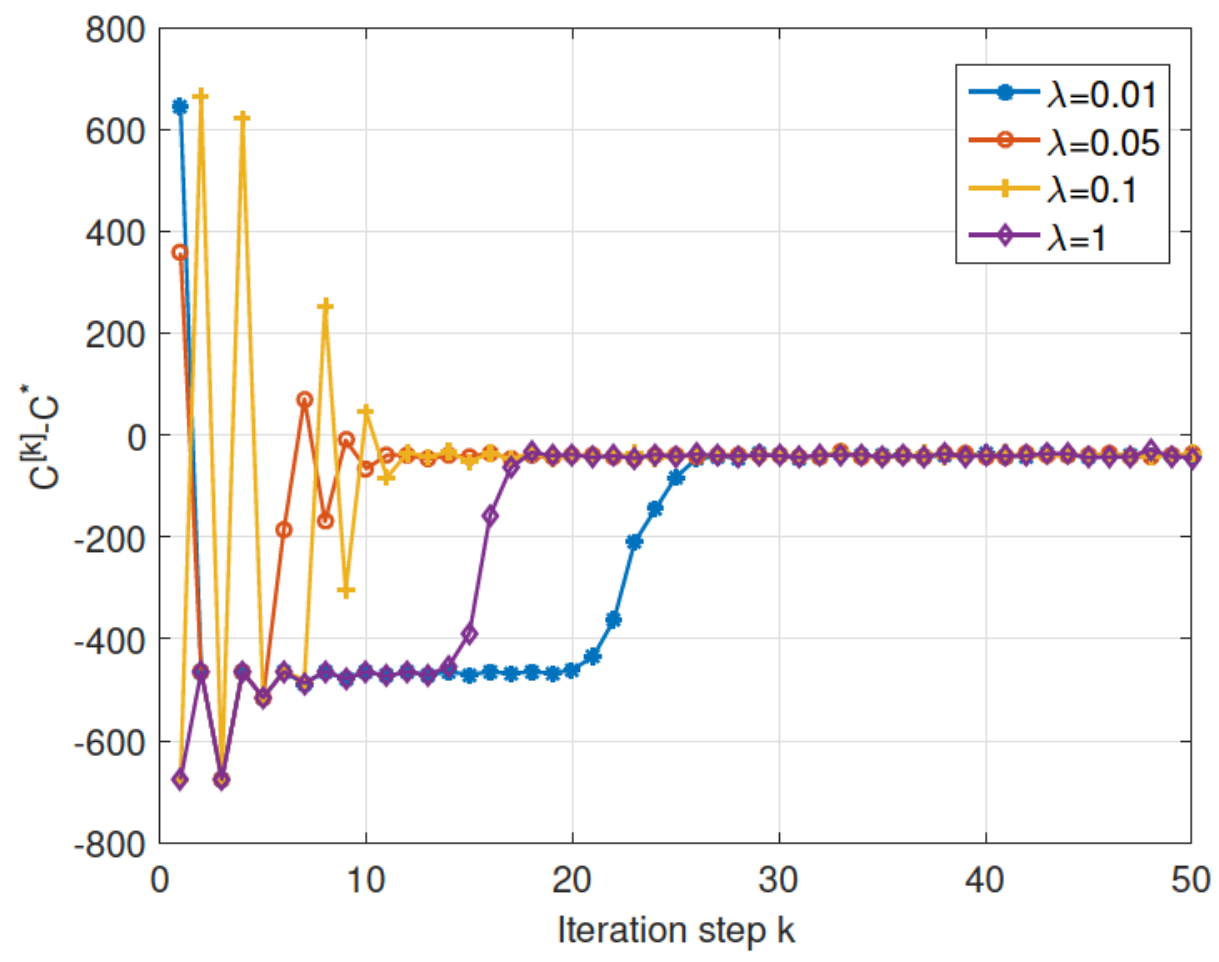

Figure 4.4: Convergence and optimality of the proposed scheme.

resource or cache resources has a tiny effect on the delay reduction. Moreover, it can be seen in Fig. 4.6b that proposed schemes with low probability surpasses the baseline when the backhaul capacity is larger than $90 \mathrm{Mbps}$. The probable reason is that the bottleneck here is behaviors of users instead of the physical resource. As shown in Fig. 4.6c, the increase of users degrades the performance of the network, because it implies that more physical resources are put to real-time flows rather than caching data. This results in less hitting events that can reduce the delay. It is interesting to observe in Fig. 4.6c that the increase of traffic load has very little effect on the cases where no dynamic caching is deployed. The intuitive reason is that since static caching is independent of traffic load. 


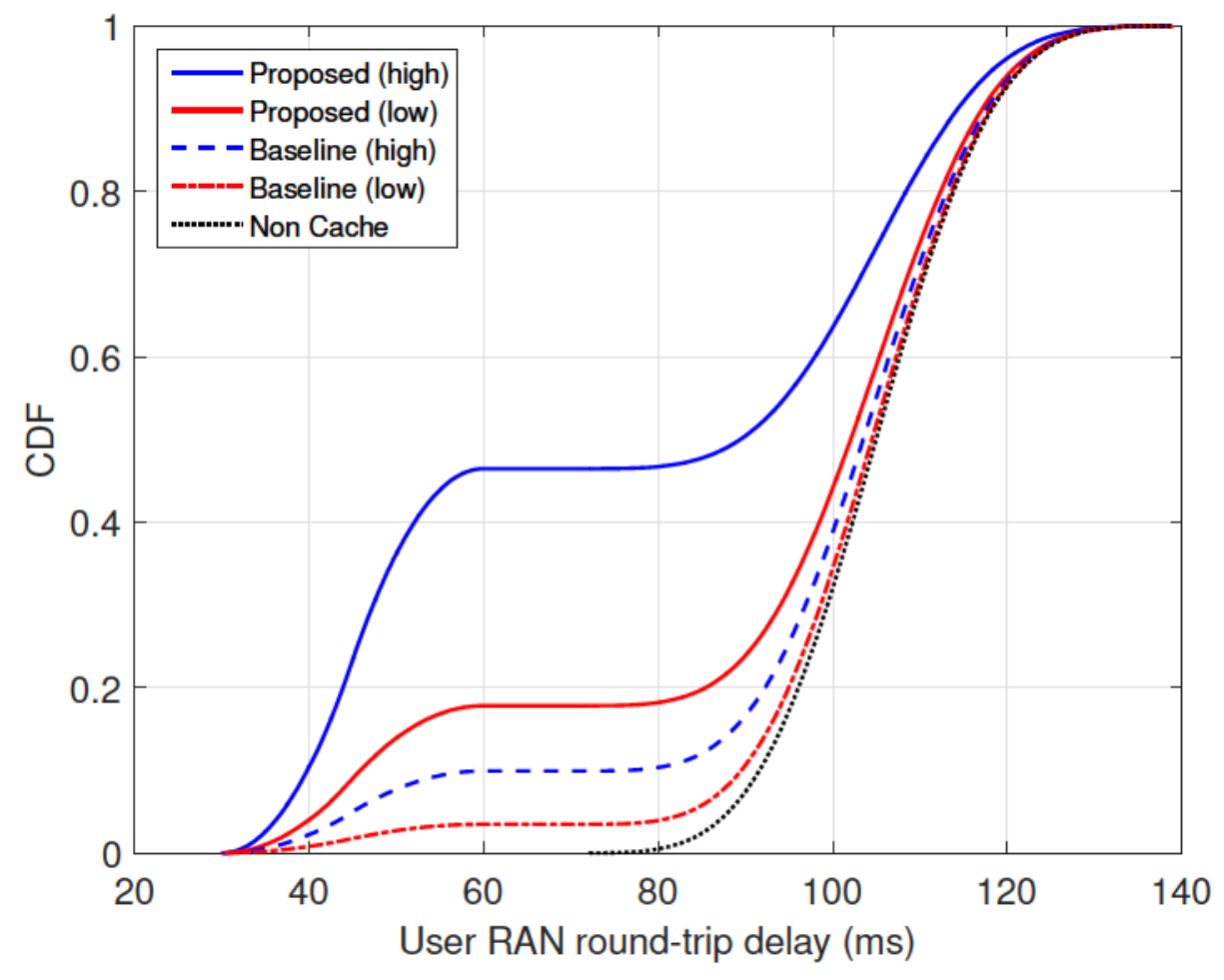

Figure 4.5: CDF of round trip delay (backhaul and RAN only) of users.

\section{QoE Guarantee}

In addition to the mean delay, it is also necessary to analyze the mobile MV-MOS of our proposed scheme. As shown in Fig. 4.7a, our proposed scheme satisfies the QoE requirements. Specifically, by putting more users into the network, the vMOS requirements are guaranteed even it has a slight decrease. Obviously, as shown in the other two figures, the boost of physical resource provides the better situation where degradation would not happen. Therefore, as mentioned Section 4.3.1, the proposed proactive caching does not undermine the video quality.

\subsubsection{Utilization}

In this subsection, the utilization of caching resource and backhaul are tested in terms of hitting events, hitting rate and backhaul load. 


\section{Caching Resource}

The hit ratio is widely adopted as the performance metric to evaluate the caching mechanisms [110]. Since our first baseline does not consider in-network caching scheme, consequently, we omit the no-caching scheme in this subsection and illustrate the average hit ratio of the cached contents of the other four schemes in our simulations in Fig. 4.8. Due to the dynamic caching operations, the proposed scheme improves the average hit ratio performance by around $30 \%$ compared to the fixed caching scheme when the probability is high, and by around $10 \%$ at most if the probability is lower. In Fig. 4.8a, it is observed that the hitting ratio of both two proposed cases is decreasing with the total number of users because more users higher the load of backhaul used for streaming videos.

Another performance measurement metric, total hitting events, also can be used to evaluate the utilization of cache resource at each BS. Fig. 4.9 shows the average total hitting within one hour per BS. Obviously, our proposed schemes show better performance than passive caching schemes. Moreover, with increasing the number of users in the networks, the total cache hitting of fixed caching is increasing due to the larger amount of users boosts the opportunities. Besides network load, with the increase of backhaul capacity of BSs and the cache capacity of each BS, the average hitting events increase significantly by deploying the dynamic caching. The reason is the same as that we mentioned in the analysis of the delay.

\section{Backhaul Resource}

In this experience, we compare the (normalized) backhaul traffic load of video streaming with different schemes in Fig. 4.10 with different parameters. In Fig. 4.10, we can see no-caching scheme takes the most backhaul load while all other four caching 
schemes can alleviate the backhaul. Specifically, with varying network status (network load, backhaul capacity and cache size), dynamic caching schemes cost more backhaul as they replace the caches more frequently than fixed caching schemes. Furthermore,the two caching cases (dynamic and fixed) of high probability save more backhaul. Apparently, higher number of users leads higher backhaul load shown in Fig. 4.10a and more physical resources give more flexible backhaul pressure. shown in Fig. 4.10b and Fig. 4.10c.

\subsection{Conclusions}

In this chapter, we jointly studied radio resource allocation, dynamic caching and adaptive video resolution selection for cache-enabled SDWNs. We proposed a polynomial time algorithm to solve the joint problem and a signaling exchange mechanism for enabling dynamic caching in SDWNs. We took the dynamic caching and adaptive video resolution selection into account of the flow control problem in SDWNs and formulate a joint problem. Then, we transferred this problem by relaxation to a convex problem that could be solved efficiently, and an algorithm was designed. To implement our proposed algorithm into practical SDWNs, a signaling exchange diagram was designed. Simulation results were presented to show that the performance of our proposed scheme can improve the QoE in terms of delay and video quality as well as efficiency of physical resource utilization. 


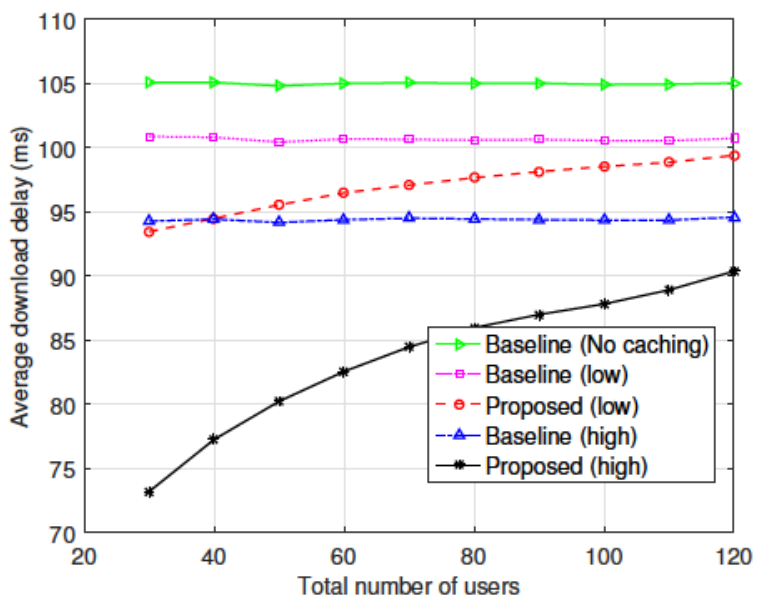

(a) Network load

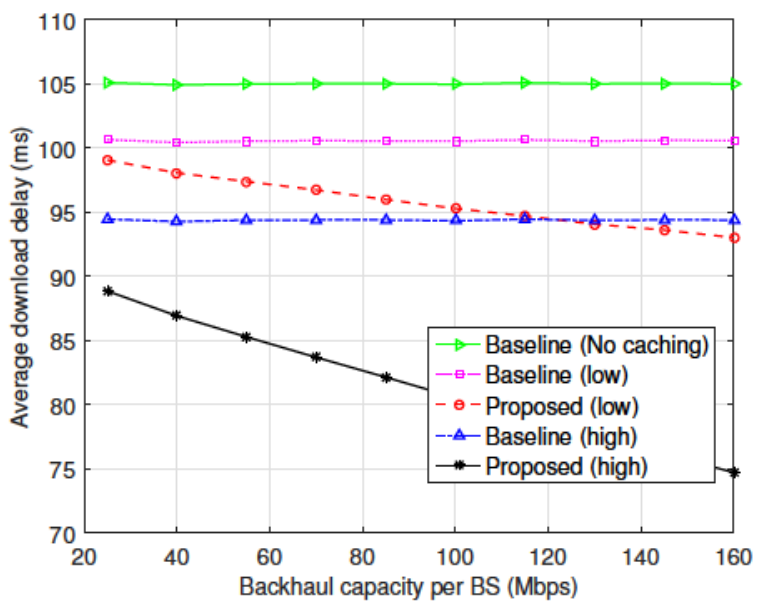

(b) Backhaul capacity

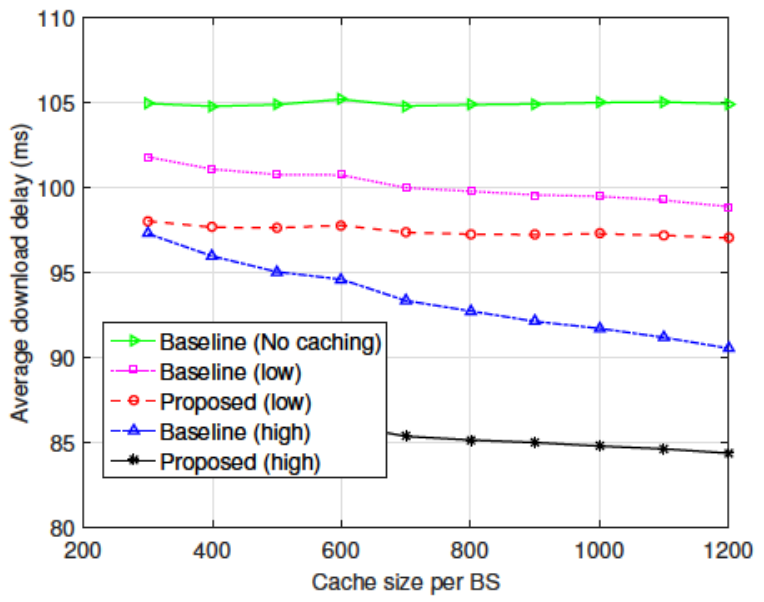

(c) Cache storage size

Figure 4.6: Average mobile network delay with different network setups. 


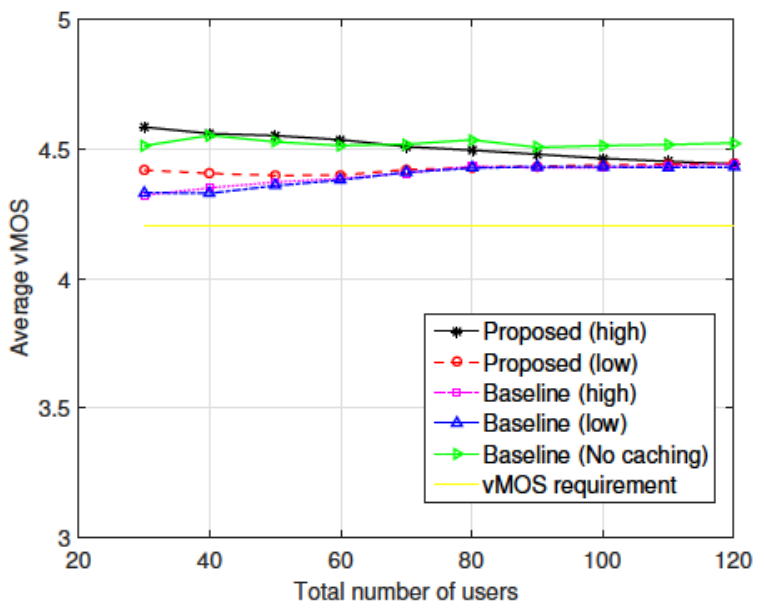

(a) Network load

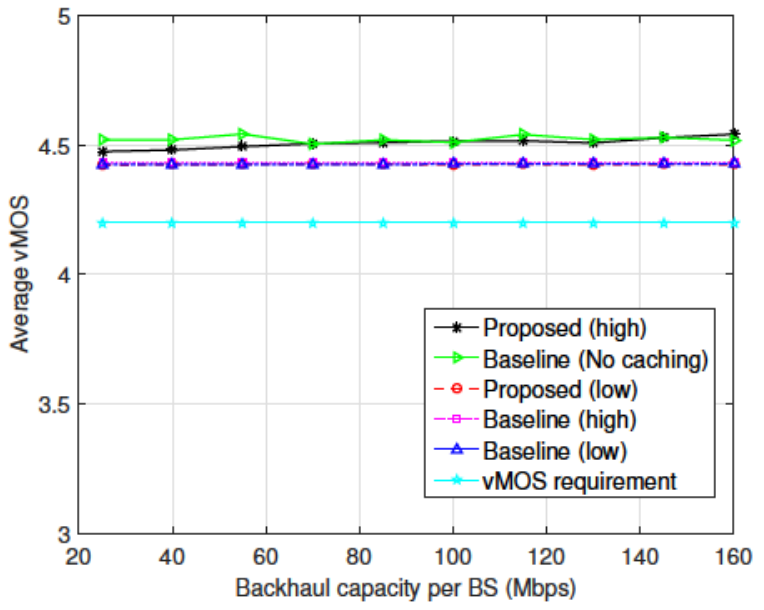

(b) Backhaul capacity

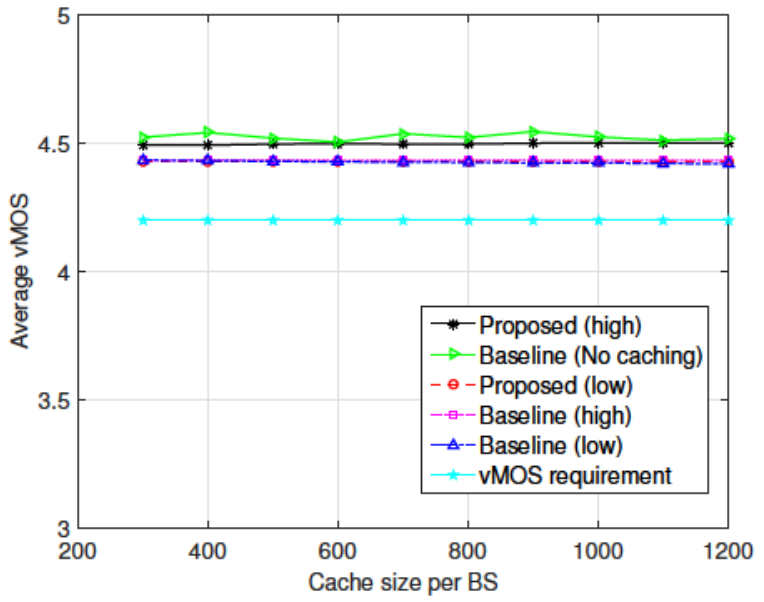

(c) Cache storage size

Figure 4.7: Average vMoS with different network setups. 


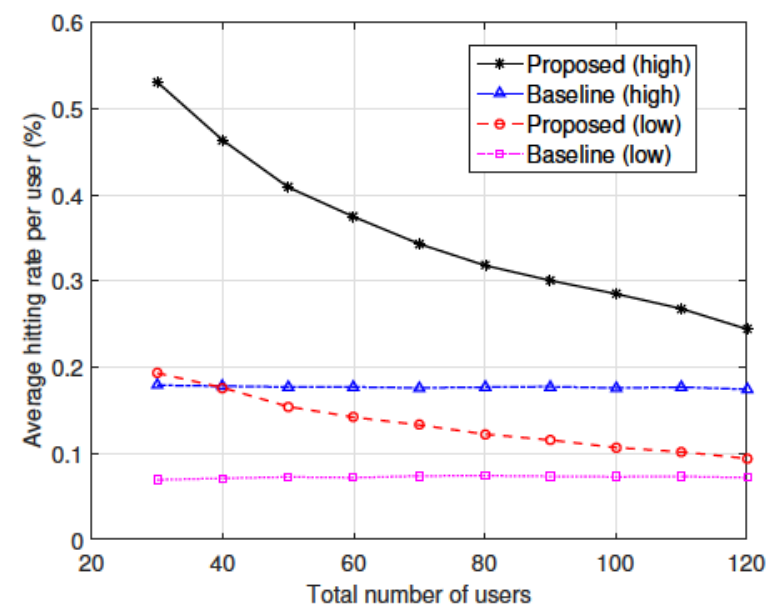

(a) Network load

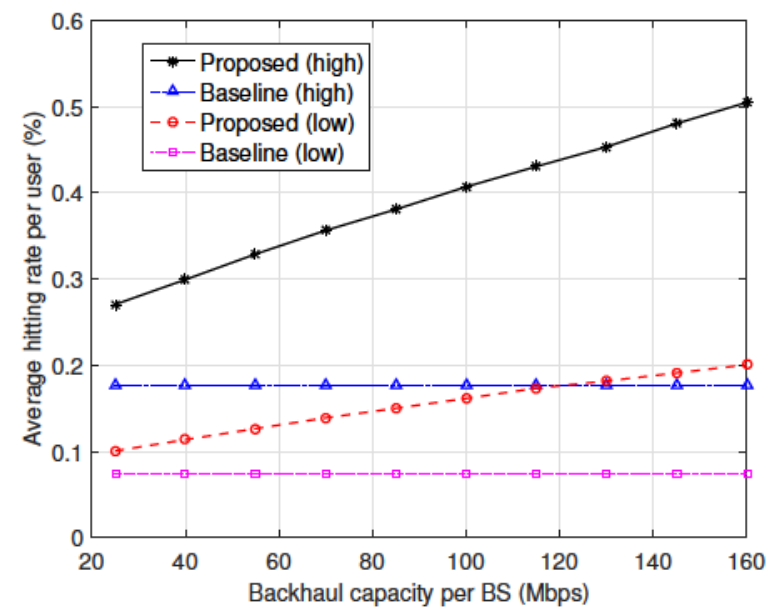

(b) Backhaul capacity

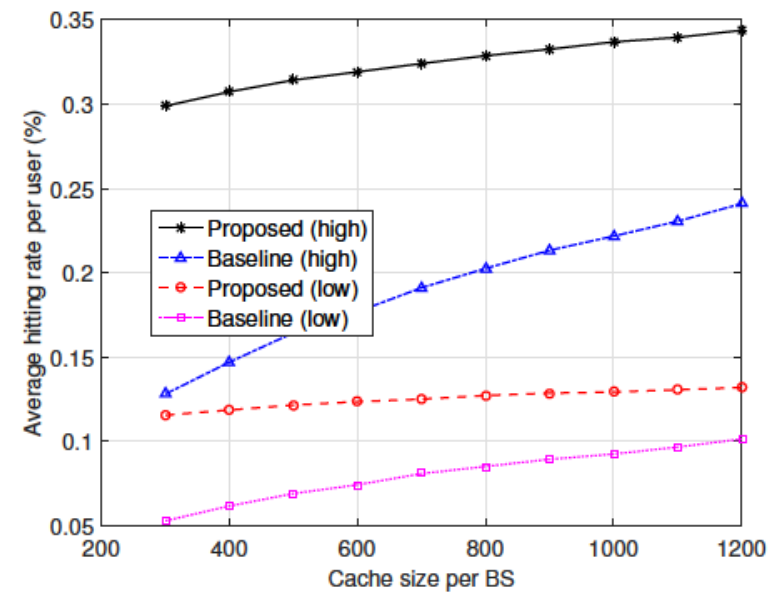

(c) Cache storage size

Figure 4.8: Average hit ratio with different network setups. 


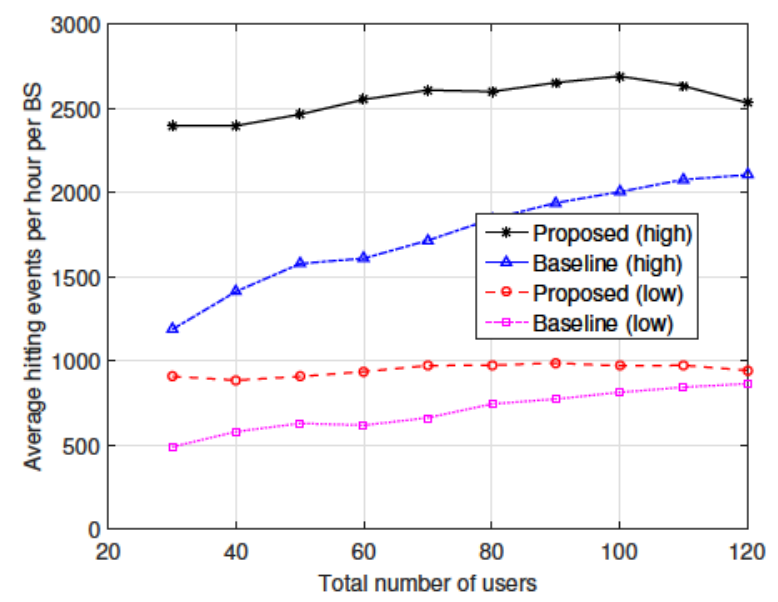

(a) Hitting events

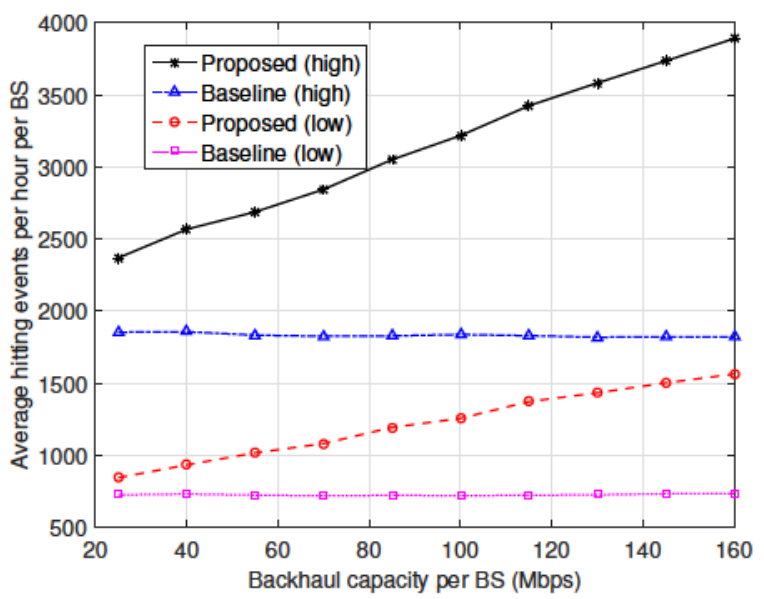

(b) Hitting rate

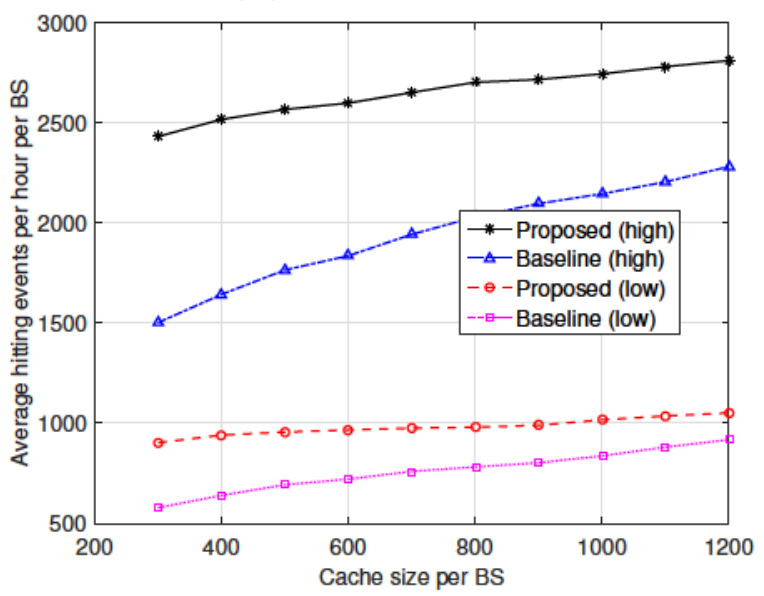

(c) Hitting rate

Figure 4.9: Average hitting events with different network setups. 


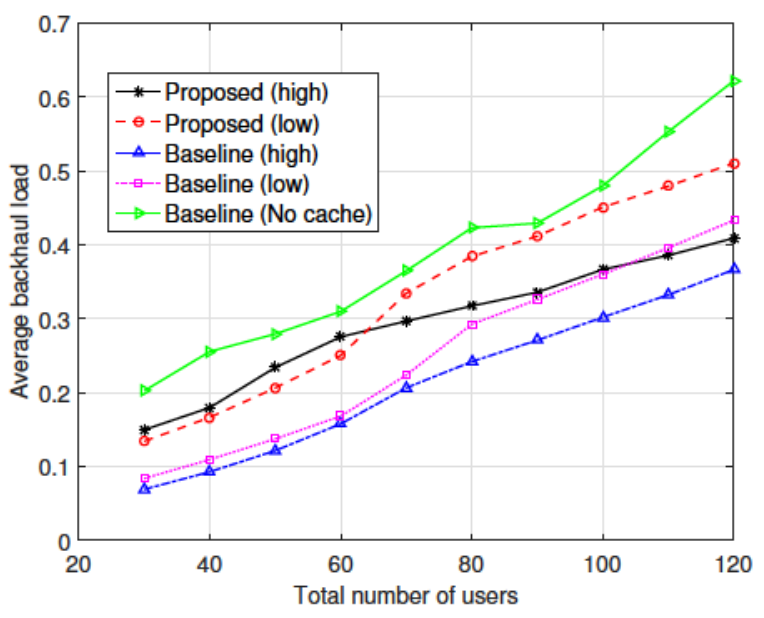

(a) Hitting events

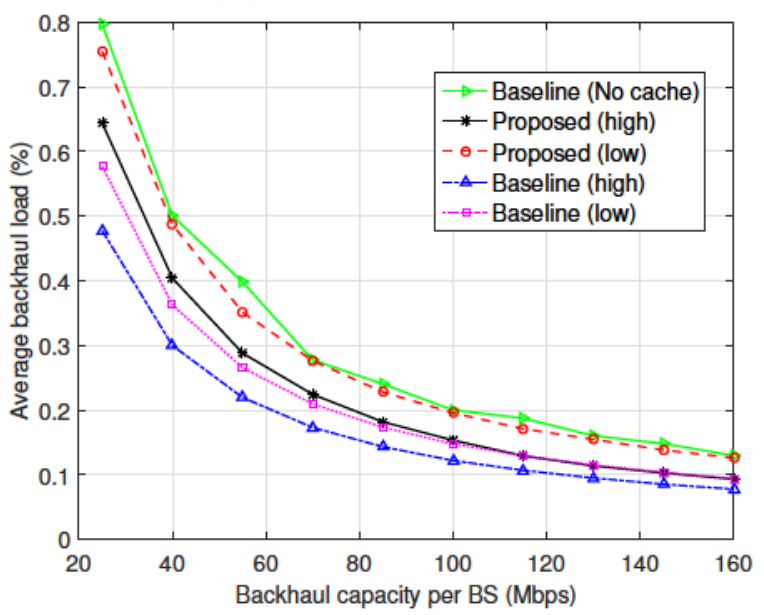

(b) Hitting rate

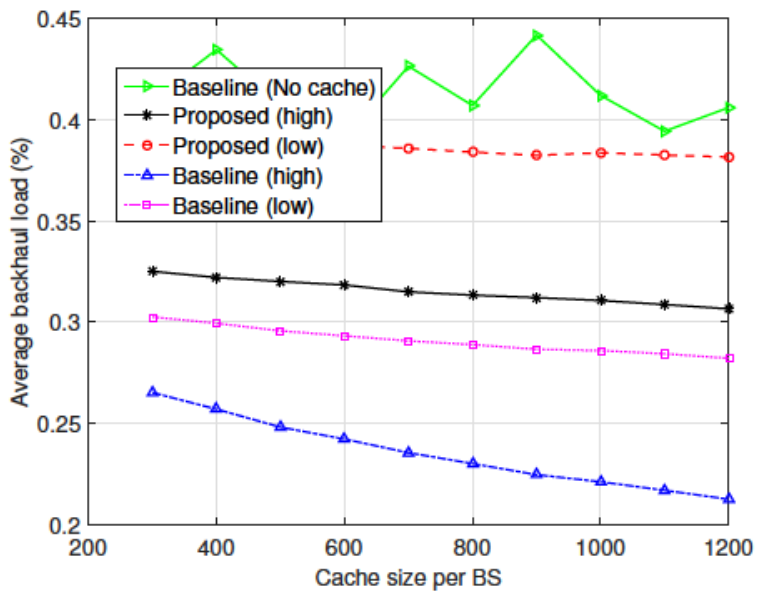

(c) Hitting rate

Figure 4.10: Average backhaul load with different network setups. 


\section{Chapter 5}

\section{Enhancing Video Rate Adaptation in}

SDWNs

\subsection{Introduction}

With the programmability, SDN is considered as a promising candidate to enhance traffic engineering, which is a key component in communication networks [33]. Generally speaking, traffic engineering is used to optimize the network and provision services with requirements by directing traffic in networks [33,34]. The success of the utilizing SDWNs for traffic engineering depends critically on our ability to jointly provision the backhaul and RANs for the traffic [32, 34, 42].

MEC has attracted great interest recently as computational resources are moved closer to users, which can efficiently improve QoS for applications that require intensive computations (e.g., video processing and tracking) [6]. With the widely employed HTTP adaptive streaming, such as Dynamic Adaptive Streaming over HTTP (DASH) (e.g., Google and Netflix), the video client can request the proper quality level adaptively according to the network throughput. However, the slow reaction of current protocols (e.g., DASH) to the fluctuated mobile network and higher requirements of QoE challenge the design of next generation mobile networks. To address this 
problem, MEC that deploys computing servers at BSs of the RAN can proactively optimize the delivery of videos by transcoding videos to desired qualities according to network conditions $[15,40,44]$.

Although some works have been done on SDWNs, in-network caching and MEC separately, jointly considering these new technologies to enhance the video service has been largely ignored in the existing research. In this chapter, we jointly consider SDWNs, in-network caching and MEC to enhance the video service in next generation mobile networks. Specifically, in the proposed framework, a SDN controller is deployed to steer the bandwidth provisioning and traffic paths selection while assisting nodes to perform edge computing and video quality selection. In addition, popular videos can be stored at caches of network nodes (e.g., router and BSs). We design an efficient mechanism that jointly considers network-assisted video rate adaptation, bandwidth provisioning in traffic engineering, and computing resource scheduling in MEC.

The distinctive technical features of this chapter are listed as follows:

- With the objective of maximizing the mean U-vMOS of a HetNet comprised with MBSs, SBSs and multiple users, an optimization problem is formulated. The limited backhaul capacity and computing capability of each network node are considered.

- Due to the coupling of video data rate, computing resource, and traffic engineering (bandwidth provisioning and paths selection), the problem becomes intractable in practice. Thus, we utilize dual-decomposition method to decouple those three sets of variables. By this decoupling, video rate adaptation is performed at users with network assistants. End nodes are able to schedule computing resource independently. Traffic engineering is performed by the SDN controller and BSs. 
- To address the challenges of dynamic change of network status and the drawbacks caused by the frequent exchange of information, we design a decentralized algorithm based on ADMM [111] to solve the traffic engineering problem.

- Extensive simulations are conducted with different system configurations to show the effectiveness of the proposed scheme.

The rest of this chapter is organized as follows. Section 5.2 introduces the system model and formulates the presented problem. Section 5.3 describes the proposed algorithms and the corresponding analysis. Simulation results are discussed in Section 5.4. Finally, we conclude this study in Section 5.5.

\subsection{System Model and Problem Formulation}

In this section, we present the system model of video streaming, mobile network, MEC and caching. The problem is formulated after the related assumptions are given. Other notations are summarized in Table 5.1.

\subsubsection{Network Model}

\section{Video streaming model}

We assume that users watch streaming videos selected from a source library $\mathcal{F}$ (e.g., Youtube or Netflix) and each video streaming is served by one data flow. For simplicity, one user can only watch one video at the same time, which leads us to use the notation $i$ to index the flow and the corresponding user. Each video file $f \in \mathcal{F}$ is encoded at a finite number of different quality levels (resolutions) $q \in\{1, \ldots, Q\}$, which is similar to DASH service. We assume that each level $q$ requires a minimum data rate $v_{q}$ (bps) to support smooth playback. Practically, $v_{q}$ depends on video 
Table 5.1: Notations of Chapter 5.

\begin{tabular}{|c|c|}
\hline Notation & Definition \\
\hline $\mathcal{F}$ & the set of video (library) \\
\hline$f$ & videos \\
\hline$q$ & video quality level (resolution) indicator \\
\hline$Q$ & highest video quality level \\
\hline$v_{q}$ & minimum required transmission data rate of the resolution $q$ \\
\hline$s_{q}$ & the U-vMOS of the resolution $q$ \\
\hline$W(\mathrm{~Hz})$ & total spectrum bandwidth \\
\hline $\mathcal{N}$ & the set of network nodes (e.g., GWs MBSs, SBSs, users) \\
\hline $\mathcal{J}$ & the set of mobile BS nodes (e.g., MBSs, SBSs) \\
\hline $\mathcal{I}$ & the set of UEs \\
\hline $\mathcal{L}$ & the set of links \\
\hline $\mathcal{L}^{w l}$ & the set of wireless links \\
\hline $\mathcal{L}^{w d}$ & the set of wired links \\
\hline$l$ & directed link \\
\hline$n_{l}$ & the source node of the link $l$ \\
\hline$m_{l}$ & the destination node of the link $l$ \\
\hline$B_{l}$ & data rate capacity of the wired link $l$ \\
\hline$\gamma_{l}$ & received spectrum efficiency of the wireless link $l$ \\
\hline$g_{n_{l} m_{l}}$ & large scale channel gain of the wireless link $l$ \\
\hline$p_{n_{l}}(\mathrm{Watt} / \mathrm{Hz})$ & the normalized transmission power on link $l$ \\
\hline$\sigma_{0}(\mathrm{Watt} / \mathrm{Hz})$ & noise power spectrum density \\
\hline $\mathcal{F}_{j}$ & the set of video (library) stored at the node $j$ \\
\hline$h_{i j}, v_{i j}$ (bps) & hitting event indicator between the flow (user) $i$ and the node $j$ \\
\hline$C_{j}(\mathrm{bps})$ & computing capacity of MEC server \\
\hline$x_{q i}$ & the resolution indicator of the user $i$ \\
\hline $\mathcal{P}_{i}$ & the path set of the user $i$ \\
\hline $\mathcal{P}_{i j}$ & the path set starting from the node $j$ \\
\hline$p_{i j}^{k}$ & the $k$-th path of the flow $i$ starting from the node $j$ \\
\hline$r_{i j}^{k}$ & the data rate of path $p_{i j}^{k}$ \\
\hline$y_{i j}$ & the computing task assignment indicator \\
\hline$\Pi_{r}, \Pi_{x}, \Pi_{y}$ & local feasible sets \\
\hline$\mu, \lambda, \nu, \varpi$ & dual variables \\
\hline$z^{\lambda}, z^{\mu}$ & sub-gradients \\
\hline$\tau$ & the length of iteration step \\
\hline$[t]$ & the iteration step \\
\hline$\check{r}_{i j}^{k}, \check{r}_{i j}^{k, l}, \hat{r}_{i j}^{k}$ & local version of $r_{i j}^{k}$ \\
\hline$\rho$ & the predefined augmented Lagrangian parameter \\
\hline
\end{tabular}


coding schemes and the video content, which are varying with time. Nevertheless, since the purpose of this dissertation is to maximize the mobile network performance dynamically, we can consider the required data rate is a fixed value $v_{q}$ when we do the scheduling.

To evaluate the gain of the video quality, the measure of each video quality $q$ is defined as $s_{q}$. In this chapter, to adapt ubiquitous HD videos in next generation mobile networks, we refer the measure to U-vMOS proposed in $[38,39]$. As shown in Table 4.5, $q$ denotes the level of an available resolution shown in the second column. Elements in the third column of Table 4.5 are minimum bandwidth (data rate) requirements $v_{q}$ to guarantee the corresponding resolutions. The fourth column of Table 4.5 is the U-vMOS of dedicated video resolution, where higher values represent better satisfaction from users.

\section{Network and communication model}

In this chapter, as shown in Fig. 5.1, we consider a mobile edge computing and caching (MECC)-enabled HetNet with the backhaul network and the RAN. The backhaul network is assumed to be a mesh network connecting SBSs, MBSs and Gateways (GWs) by wired links with fixed capacities. Users are connected to BSs with wireless channels sharing total $W(\mathrm{~Hz})$ radio spectrum resource. This considered network is modeled by a directed graph $G(\mathcal{N}, \mathcal{L}) . \mathcal{N}$ includes network nodes (GWs and BSs) formed a set $\mathcal{J}$ and users formed a set $\mathcal{I}$. $\mathcal{L}$ comprised with wired and wireless links denoted by sets $\mathcal{L}^{w d}$ and $\mathcal{L}^{w l}$, respectively. $m_{l} \in \mathcal{N}$ and $n_{l} \in \mathcal{N}$ are used to denote the destination node and the source node of link $l$, respectively.

If link $l$ is a wired link, it is assumed to provide a fixed bandwidth capacity $B_{l}$. If link $l$ is a wireless link, the capacity depends on the ratio of the radio resource that the network allocates to this link. In this chapter, to simply our analysis, we do not 


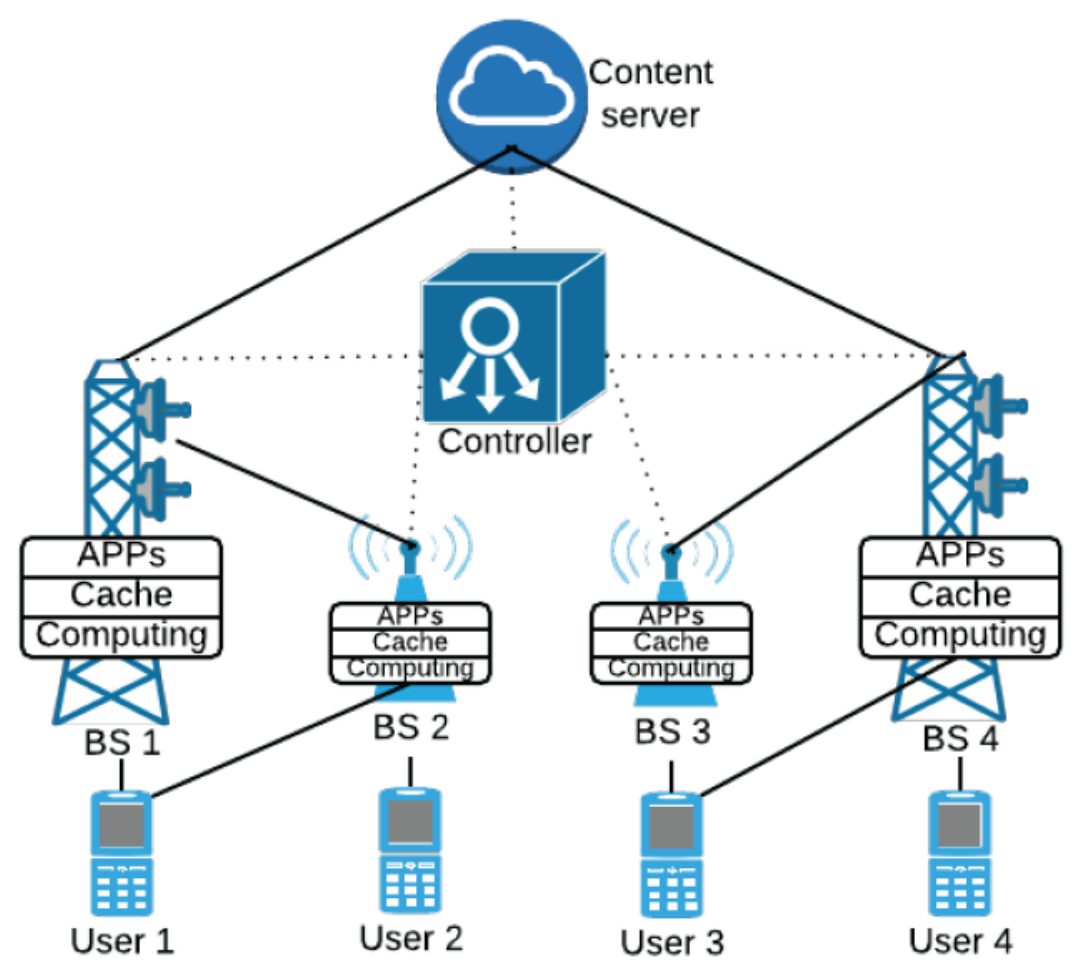

Figure 5.1: MEC-enabled software-defined mobile network.

consider any advanced interference management and power allocation schemes. Thus, by using the Shannon bound, the spectrum efficiency of wireless link $l$ is defined as

$$
\gamma_{l}=\log \left(1+\frac{g_{n_{l} m_{l}} p_{n_{l}}}{\sigma_{0}}\right),
$$

where $g_{n_{l} m_{l}}$ is the large-scale channel gain that includes pathloss and shadowing between the transmission node $n_{l}$ (the source of link $l$ ) and the receiving node $m_{l}$ (the destination of link $l$ ). We deploy the same model used in [81] to calculate the pathloss and apply shadowing. $p_{n_{l}}$ (Watt/Hz) is the normalized transmission power on link $l$. The fixed equal power allocation mechanism is used, which means transmission power $p_{n l}$ is the same for all frequencies. $\sigma_{0}$ is the power spectrum density of additive white 
Gaussian noise. Accordingly, the achievable data rate capacity of link $l$ is $R_{l}=W \gamma_{l}$.

Each data flow can be spitted to multiple paths as the user is assumed to be served by multiple BSs through BS cooperation [32] or multistream carrier aggregation. Moreover, demanded videos can be potentially retrieved from any nodes (GWs, BSs or the source server) where matched data are found, which means each user can download the data of video from different places. For example, in Fig. 5.1, the data flow of user 1 is split to two paths where one is from content source server to MBS 1 then to users 1 and another is directly from SBS 2.

\section{Caching and Computing Model}

The network is equipped with caching and computing functions on network nodes. We assume a subset $\mathcal{F}_{j}$ of $\mathcal{F}$ is stored at node $j$. It should be noted that node $j$ always caches the highest quality $Q$ of video file $f$ so that it can be transcoding to a lower quality. As we mentioned above, if video file $f_{i}$ demanded by user $i$ is found at node $j$, namely $f_{i} \in \mathcal{F}_{j}$, node $j$ becomes a candidate source. To indicate a hitting event between user $i$ and node $j$, we define $h_{i j}=1$ and $v_{i j}=v_{i}$ if $f_{i} \in \mathcal{F}_{j} . v_{i j}=v_{i}$ means the video requested by user $i$ can be potentially fully provided by node $j$ and $h_{i j}=1$ means a successful hitting event. If $f_{i} \notin \mathcal{F}_{j}, h_{i j}=+\infty$ that means there is no hitting event (infinite resource is required to process this video) and $v_{i j}=0$ means node $j$ cannot response the video request $i$.

If $h_{i j}=1$ and node $j$ is selected as one of source nodes of flow $i$, the video data needs to be transcoded to the required quality level except that the highest quality level is selected. However, unlike the powerful computing resource at the source server (e.g., the data center), due to the computing resource at each node, limited tasks can be activated at the same time. Similar to [40], we define the maximum mobile computing capacity as the number of encoded bits that can be processed per 
second, denoted by $C_{j}$ (bps). For example, a 500 Mbps computing capacity means 20 concurrent video processing tasks are allowed.

\subsubsection{Problem Formulation}

The network-assisted rate adaptation problem in a SDWN with MECC can be stated and formulated as follows.

\section{Video rate adaptation}

The purpose of this considered problem is to find an optimal video quality level for each user with considering network resources and the cached video distribution. We define a binary variable $x_{q i} \in\{0,1\}$ as the resolution indicator of user $i$. Specifically, if the $q$-th resolution of the video is selected by user $i, x_{q i}=1$; otherwise, $x_{q i}=0$.

\section{Path Selection and Resource Allocation}

To support video services demanded by users, an optimal path set for all data flows should be found by solving the proposed algorithm. Denote $\mathcal{P}_{i}$ as a path set including all candidate paths for user $i$ and $\mathcal{P}_{i j}$ as a subset of $\mathcal{P}_{i}$ including all candidate paths starting from node $j$. A path $p_{i j}^{k} \in \mathcal{P}_{i}$ means the $k$-th path of flow $i$ starting from node $j$ and the corresponding data rate of this path is denoted by $r_{i j}^{k}$ if $p_{i j}^{k}$ is selected. Thus the achievable data rate of flow $i$ is $\sum_{p_{i j}^{k} \in \mathcal{P}_{i}} r_{i j}^{k}$ that is the aggregated rate of all selected paths.

\section{Computing Scheduling}

As we mentioned in above, the computing resource on each node needs to be scheduled to video transcoding tasks. Thus, we define a binary variable $y_{i j} \in\{0,1\}$ as the computing task assignment indicator. If $y_{i j}=1$, node $j$ is able to trans-code the 
video demanded by user $i$ to a desired quality level; otherwise, the video data cannot be retrieved from node $j$.

\section{Proposed SDN-assisted Rate Adaptation Problem}

To improve the whole network utility by maximizing the overall mean U-vMOS, the SDN controller performs traffic engineering to assist users adaptively selecting optimal video quality levels. Thus, the proposed problem can be formed as follows.

$$
\max _{\mathbf{X}, \mathbf{R}, \mathbf{Y}} \quad U(\mathbf{X})=\frac{1}{|\mathcal{I}|} \sum_{i \in \mathcal{I}} \sum_{q=1}^{Q} s_{q} x_{q i}
$$

subject to

$$
\begin{array}{r}
r_{i j}^{k} \in \Re^{+}, \forall i, j, k, \\
x_{q i}, y_{i j} \in\{0,1\}, \forall q, i, j, k, \\
\sum_{q=1}^{Q} x_{q i}=1, \forall i \in \mathcal{I}, \\
\sum_{i \in \mathcal{I}} y_{i j} h_{i j} c_{i} \leq C_{j}, \forall j \in \mathcal{J},
\end{array}
$$

Flow conservation law:

$$
\sum_{p_{i j}^{k} \in \mathcal{P}_{l}} r_{i j}^{k} \leq B_{l}, \forall l \in \mathcal{L}^{w d}
$$




$$
\sum_{l \in \mathcal{L}^{w l}} \frac{\sum_{p_{i j}^{k} \in \mathcal{P}_{l}} r_{i j}^{k}}{\gamma_{l}} \leq W,
$$

$$
\sum_{p_{i j}^{k} \in \mathcal{P}_{i}} r_{i j}^{k}=\sum_{q} d_{q i} x_{q i}, \forall i \in \mathcal{I}
$$

Content constraint:

$$
\sum_{p_{i j}^{k} \in \mathcal{P}_{i j}} r_{i j}^{k} \leq v_{i j}\left(x_{Q i}+y_{i j}\right), \forall i \in \mathcal{I}, j \in \mathcal{J}, k \in \mathcal{K}
$$

where $\left\{x_{q i}\right\},\left\{y_{i j}\right\}$ and $\left\{r_{i j}^{k}\right\}$ are elements of $\mathbf{X}, \mathbf{Y}$ and $\mathbf{R}$, respectively. Constraint (5.2c) reflects that only one resolution level can be selected for one user. The computing capacity on each node $j$ is specified by the constraint (5.2d) where $c_{i}$ (bps) is the computing resource required for transcoding video $f_{i}$. The flow conservation law (FCL) of traffic engineering is claimed by constraints (5.2e) and (5.2f) where $\mathcal{P}_{l}$ is the set of paths that pass link $l$. (5.2e) means the allocated data rate of link $l$ for all passing path should be less than the link capacity. As the radio resource is shared by the whole RAN, (5.2f) enforces that the total allocated spectrum cannot exceed the available spectrum bandwidth. The demand constraint for every video flow is given by the $(5.2 \mathrm{~g})$. The constraint $(5.2 \mathrm{~h})$ requires that any candidate path starting from node $j$ can be selected only when it has the content in the cache $\left(h_{i j}=1\right)$ and the computing resource is assigned to transcode this content $\left(y_{i j}=1\right)$ or the highest quality is selected $\left(x_{Q i}=1\right)$.

Unfortunately, problem (5.2) is difficult to solve and implement based on the following observations: 
- The mix integer variables result in the problem a mix-integer linear problem (MILP) that generally is NP-complete.

- The complexity of solving (5.2) by using greedy or genetic methods will increase significantly with the increase of the number of users and (or) BSs.

- Video resolution, path selection, and resource scheduling are decided by different layers, network nodes and perform in different time scales.

- The necessary exchange of local information about the network and links affects the performance as overheads are introduced.

In the next section, we will develop a scheme to solve problem (5.2) so that a near-optimal solution can be found.

\subsection{Proposed SDN-assisted Video Rate Adapta- tion with MECC}

In this section, dual-decomposition method is deployed to simplify problem (5.2). An ADMM-based algorithm is designed to perform traffic engineering. An example of the proposed scheme is presented as well.

\subsubsection{Problem Decomposition}

This dissertation aims to give an efficient scheme to help video clients to select appropriate video resolutions while conducting resource scheduling to provision bandwidth and process video data. Observed from problem (5.2), the network tries to maximize the utility gained by video resolution. However, the video resolution is selected by the video client on each user. Moreover, MEC resource is assigned by each node based on 
the video distribution and requests, which leads the network hard to steer the traffic. Thus, the network needs to transfer some information to assist users and nodes when they perform video selection and processing so that the optimal network utility can obtained.

Those features of (5.2) motivate us to adopt dual-decomposition method so that the video quality selection and the MEC scheduling can be separated from the bandwidth provisioning of traffic engineering performed by the SDN controller. Before we form the partial Lagrangian function for problem (5.2), let us define independent local feasible sets $\Pi_{r}, \Pi_{x}$, and $\Pi_{y}$ for variables $\mathbf{R}, \mathbf{X}$ and $\mathbf{Y}$, respectively. Those feasible regions only subject to constraints that include one type of variables, which are shown as

$$
\begin{aligned}
& \Pi_{r}=\left\{\left\{r_{i j}^{k}\right\} \mid \Re^{+},(5.2 e),(5.2 f)\right\} . \\
& \Pi_{x}=\left\{\left\{x_{q i}\right\} \mid\{0,1\},(5.2 c)\right\} . \\
& \Pi_{y}=\left\{\left\{y_{i j}\right\} \mid\{0,1\},(5.2 d)\right\} .
\end{aligned}
$$

Fortunately, the coupled constraints are $(5.2 \mathrm{~g})$ and $(5.2 \mathrm{~h})$. Thus, by relaxing constraints (5.2g) and (5.2h) with dual variables $\left\{\mu_{i}\right\}$ and $\left\{\lambda_{i j}\right\}^{1}$, the Lagrangian can be

\footnotetext{
${ }^{1}$ Dual variables can be interpreted as costs of bandwidth and computing
} 
shown as:

$$
\begin{array}{rl}
\max _{\mathbf{X}, \mathbf{R}, \mathbf{Y}} & U(\mathbf{X})+\sum_{i \in \mathcal{I}} \mu_{i}\left[\sum_{p_{i j}^{k} \in \mathcal{P}_{i}} r_{i j}^{k}-\sum_{q=1}^{Q} v_{q i} x_{q i}\right] \\
& -\sum_{i \in \mathcal{I}, j \in \mathcal{J}} \lambda_{i j}\left[\sum_{p_{i j}^{k} \in \mathcal{P}_{i j}} r_{i j}^{k}-v_{i j}\left(x_{Q i}+y_{i j}\right)\right]
\end{array}
$$

s.t. $\mathbf{X} \in \Pi_{x}, \mathbf{Y} \in \Pi_{y}, \mathbf{R} \in \Pi_{r}$.

Thus, the original problem has been separated to two levels of optimization that are higher level for updating dual variables and low level for finding dual functions [112]. Accordingly, the dual problem (DP) then is:

$$
\text { DP : } \min _{\mu \in \mathbb{R}, \lambda \in \mathbb{R}^{+}} D(\mu, \lambda)=g_{x}(\mu, \lambda)+g_{r}(\mu, \lambda)+g_{y}(\lambda)
$$

where $g_{x}(\mu, \lambda), g_{r}(\mu, \lambda)$, and $g_{y}(\lambda)$ are dual functions obtained as the maximum value of the Lagrangians solved in following problems (5.8), (5.9) and (5.10) for given $\left\{\mu_{i}\right\}$ and $\left\{\lambda_{i j}\right\}$.

$$
\begin{aligned}
& g_{x}(\mu, \lambda)=\sup _{x_{q i} \in \Pi_{x}}\left\{\begin{array}{c}
U(\mathbf{X})-\sum_{i \in \mathcal{I}}^{I} \mu_{i} \sum_{q} v_{q i} x_{q i} \\
+\sum_{i \in \mathcal{I}, j \in \mathcal{J}} \lambda_{i j} v_{i j} x_{Q i}
\end{array}\right\}, \\
& g_{r}(\mu, \lambda)=\sup _{r_{i j}^{k} \in \Pi_{r}}\left\{\sum_{i \in \mathcal{I}} \sum_{p_{i j}^{k} \in \mathcal{P}_{i}}\left(\mu_{i}-\lambda_{i j}\right) r_{i j}^{k}\right\}, \\
& g_{y}(\mu, \lambda)=\sup _{y_{i j} \in \Pi_{y}}\left\{\sum_{i \in \mathcal{I}, j \in \mathcal{J}} \lambda_{i j} v_{i j} y_{i j}\right\}
\end{aligned}
$$


It is observed that $D(\mu, \lambda)$ is not a differentiable function due to the binary variables and candidate path sets. Thus, we can deploy sub-gradient method to solve the dual problem (5.7). Obviously, a sub-gradient of problem (5.7) for $\lambda_{i j}$ is:

$$
z_{i j}^{\lambda}=\sum_{p_{i j}^{k} \in \mathcal{P}_{i j}} r_{i j}^{k}-v_{i j}\left(x_{Q i}+y_{i j}\right)
$$

and for $\mu_{i}$ is

$$
z_{i}^{\mu}=\sum_{p_{i j}^{k} \in \mathcal{P}_{i}} r_{i j}^{k}-\sum_{q} v_{q i} x_{q i}
$$

According to dual decomposition [109], we thus can update $\mu_{i}$ and $\lambda_{i j}$ based on:

$$
\mu_{i}^{[t+1]}=\mu_{i}^{[t]}-\tau_{\mu}^{[t]} z_{i}^{\mu}
$$

and

$$
\lambda_{i j}^{[t+1]}=\left[\lambda_{i j}^{[t]}-\tau_{\lambda}^{[t]} z_{i j}^{\lambda}\right]^{+}
$$

where $\tau_{\mu}^{[t]}$ and $\tau_{\lambda}^{[t]}$ are the length of step at iteration step $[t]$.

Thus, if we are able to solve the inner problems (5.8), (5.9) and (5.10) in each iteration, the SDN controller can update dual variables and transfer them to nodes and users to assist them to find optimal solutions of their own variables $x_{q i}, r_{i j}^{k}$, and $y_{i j}$. This SDN-assisted optimization scheme is summarized in Table 5.2. In the remaining of this section, algorithms will be given to solve problems (5.8), (5.9) and (5.10). 
Table 5.2: Algorithm for video rate adaptation and scheduling

\section{SDN-assisted video rate adaptation and resource scheduling}

1: Initialize: Set stop criteria $\epsilon$ and maximum iterative steps $T$

2: SDN controller sets initial dual variables $\lambda_{i j}^{[0]}, \mu_{i}^{[0]}$

3: Users (video clients) select initial video quality levels $\mathbf{X}$ and nodes (network function and MEC) set initial resource allocation $\{\mathbf{R}, \mathbf{Y}\}$.

4: $t=0$

5: for $t \leq T$ or $\epsilon$ is not met do

6: SDN controller broadcasts dual variables $\lambda_{i j}^{[t]}, \mu_{i}^{[t]}$ to users and nodes

7: $\quad$ Each user selects the video quality by solving (5.8) given $\lambda_{i j}^{[t]}$ and $\mu_{i}^{[t]}$

8: $\quad$ Each node schedules the computing resources by solving (5.10) given $\lambda_{i j}^{[t]}$

9: The mobile provisions the bandwidth for flows by solving (5.9) given $\lambda_{i j}^{[t]}$ and $\mu_{i}^{[t]}$

10: The SDN controller computes the sub-gradient of $\lambda_{i j}$ and $\mu_{i}$ based on (5.11) and (5.12)

11: The SDN controller updates $\lambda_{i j}^{[t+1]}$ and $\mu_{i}^{[t+1]}$ according to (5.13) and (5.14)

12: $\quad$ d) $t=t+1$

13: end for

14: Update $\{\mathbf{X}, \mathbf{R}, \mathbf{Y}\}$

\subsubsection{Video Rate Adaptation Based on Network Information}

Observe that problem (5.8) can be decoupled to users where the local problem of each user is shown as

$$
\begin{aligned}
\max _{x_{q i} \in\{0,1\}} & \sum_{q=1}^{Q} s_{q} x_{q i}-\mu_{i} \sum_{q} v_{q i} x_{q i}+\sum_{j \in \mathcal{J}} \lambda_{i j} v_{i j} x_{Q i} \\
\text { s.t. } & \sum_{q=1}^{Q} x_{q i}=1 .
\end{aligned}
$$

The above problem can be solved with effortless due to that only one quality level can be selected. Thus, each user only needs to select the level maximizing the utility.

Here we focus on the analysis of the structure of the objective so that the assistant from the SDN controller can be understood. $\mu_{i}$ represents the bandwidth cost (revenue) of user $i$ given by the network. $\mu_{i}<0$ means the network can provide more bandwidth for the user by using a revenue to push the user select higher resolution. 
However, if $\mu_{i} \geq 0$, the network may be lack of enough resource to support a higher video quality. As $\lambda_{i j}$ is always non-negative, the function here is to encourage the user to select the best quality as it is cached at node $j$ when $v_{i j} \neq 0$.

\subsubsection{Computing Resource Scheduling Based on Network In- formation}

Similar to problem (5.8), problem (5.10) also can be decoupled to each node $j$ as follows.

$$
\begin{aligned}
& \max _{y_{i j} \in\{0,1\}} \sum_{i \in \mathcal{I}} \lambda_{i j} v_{i j} y_{i j} \\
& \text { s.t. } \quad \sum_{i \in \mathcal{I}} y_{i j} h_{i j} v_{i} \leq C_{j},
\end{aligned}
$$

Obviously, this is a 0-1 knapsack problem that is usually NP-complete. Thus, BnB method or dynamic programming can be used to solve this problem, but they are both computationally intensive and might not be practical for large-scale problem. Therefore, firstly, to ease the size of this problem so that common methods can be used, we form a set $\mathcal{I}_{j}^{+}$including every user who has non-zero gain $\lambda_{i j} v_{i j}$ or finite $h_{i j}$. Formally, $\mathcal{I}_{j}^{+}:=\left\{i \mid \lambda_{i j} v_{i j}>0, h_{i j}<\infty\right\}$. It is easy to see that $y_{i j}=0$ if $i \notin \mathcal{I}_{j}^{+}$. Thus, we only need to consider users that in $\mathcal{I}_{j}^{+}$, which leads to a reduction of the problem size.

If a special case is available, this scheme can be further improved. We assume that $v_{i}=v, \forall i \in \mathcal{I}_{j}^{+}$. Then, the maximum number of tasks that node $j$ can process is $c_{j}=\left\lfloor\frac{C_{j}}{v}\right\rfloor$ where $\lfloor\cdot\rfloor$ means taking the maximum integer value that is less than $\cdot$. By knowing $c_{j}$, the MEC is able to select the best $c_{j}$ tasks from $\mathcal{I}_{j}^{+}$. In a large degree, in practice, we can consider each video has the same average rate at the same quality level. 


\subsubsection{ADMM-based Algorithms of Bandwidth Provisioning}

This problem (5.9) is easy to solve theoretically as it is a linear problem. However, wired backhaul and radio access links are involved in this problem, which leads the solution hard to achieve in practice. Thus, a decentralized method is appropriate to cope with this problem. In this subsection, we propose to use ADMM as the tool to update $r_{i j}^{k}$. ADMM can be considered as a combination of the dual decomposition and the augmented Lagrangian methods [74,113]. To apply ADMM, problem (5.9) has to be decoupled to subproblems, thus we need firstly to separate the wired part and the wireless part by introducing some local variables.

We firstly separate the wired backhaul network and the radio access network, which is similar to studies in $[32,114]$. Denote the data rate of path $p_{i j}^{k}$ decided by the wired backhaul network is $\check{r}_{i j}^{k}$ and its peer decided by the RAN is $\hat{r}_{i j}^{k}$. Furthermore, the wired backhaul network can be decoupled to links, as capacities of wired links usually are independent with each other. $\check{r}_{i j}^{k, l}$ denotes that the data rate of path $p_{i j}^{k}$ allocated by link $l$. It should be noted that $\check{r}_{i j}^{k, l}$ does not mean $p_{i j}^{k}$ passing link $l . \check{r}_{i j}^{k, l}$ can be considered the opinion for $p_{i j}^{k}$ given by link $l$. Actually, all local varialbes of $r_{i j}^{k}$ is the opinion or recommendation from one part of the whole network. By defining $\check{r}_{i j}^{k, l}$ and $\hat{r}_{i j}^{k}$, problem (5.9) can be revised as

$$
\begin{aligned}
& \max _{\check{r}_{i j}^{k, l}, \hat{r}_{i j}^{k} \in \mathbb{R}^{+}} \frac{1}{2 L^{w d}} \sum_{l \in L^{w d}} \sum_{i \in \mathcal{I}} \sum_{p_{i j}^{k} \in \mathcal{P}_{i}}\left(\mu_{i}-\lambda_{i j}\right) \check{r}_{i j}^{k, l}+\frac{1}{2} \sum_{i \in \mathcal{I}} \sum_{p_{i j}^{k} \in \mathcal{P}_{i}}\left(\mu_{i}-\lambda_{i j}\right) \hat{r}_{i j}^{k} \\
& \text { s.t. } \sum_{i \in \mathcal{I}} \sum_{p_{i j}^{k} \in \mathcal{P}_{i}} \alpha_{i j}^{k, l} \check{r}_{i j}^{k, l} \leq B_{l}, \forall l \in \mathcal{L}^{w d}, \\
& \sum_{l \in \mathcal{L}^{w l}} \frac{\sum_{i \in \mathcal{I}} \sum_{p_{i j}^{k} \in \mathcal{P}_{i}} \alpha_{i j}^{k, l} \hat{r}_{i j}^{k}}{\gamma_{l}} \leq W, \\
& \check{r}_{i j}^{l, k}=\hat{r}_{i j}^{k}, \forall i, j, k, l .
\end{aligned}
$$




\section{Table 5.3: Agorithm for bandwidth provisioning}

\section{Decentralized bandwidth provisioning via ADMM}

1: Initialize:

2: Set stop criteria $\epsilon$ and maximum iterative steps $T$

3: Set primal and dual variables $\left\{\check{r}_{i j}^{k, l}, \hat{r}_{i j}^{k}, \nu_{i j}^{k, l}\right\}$

4: $t=0$

5: for $t \leq T$ or $\epsilon$ is not met do

6: The SDN controller of the RAN broadcast $\left\{\nu_{i j}^{k, l}\right\}$ and $\left\{\hat{r}_{i j}^{k}\right\}$ to each wired link $l$

7:

8: $\quad$ Each wired link $l$ solves following problem to update $\left\{\check{r}_{i j}^{k, l}\right\}$;

$$
\begin{aligned}
\max _{\check{r}_{i j}^{k, l} \in \mathbb{R}^{+}} & \frac{1}{2 L^{w d}} \sum_{i \in \mathcal{I}} \sum_{p_{i j}^{k} \in \mathcal{P}_{i}}\left(\mu_{i}-\lambda_{i j}\right) \check{r}_{i j}^{k, l}+\sum_{i \in \mathcal{I}} \sum_{p_{i j}^{k} \in \mathcal{P}_{i}} \nu_{i j}^{k, l}\left(\check{r}_{i j}^{k, l}-\hat{r}_{i j}^{k}\right) \\
& -\frac{\rho}{2} \sum_{i \in \mathcal{I}} \sum_{p_{i j}^{k} \in \mathcal{P}_{i}}\left(\check{r}_{i j}^{k, l}-\hat{r}_{i j}^{k}\right)^{2} \\
\text { s.t. } & \sum_{i \in \mathcal{I}} \sum_{p_{i j}^{k} \in \mathcal{P}_{i}} \alpha_{i j}^{k, l} \check{r}_{i j}^{k, l} \leq B_{l}, \forall l \in \mathcal{L}^{w d},
\end{aligned}
$$

9:

Each link $l$ send $\left\{\check{r}_{i j}^{k, l}\right\}$ to the SDN controller

10: The SDN controller of the RAN solves following problem to update $\left\{\check{r}_{i j}^{k, l}\right\}$;

$$
\begin{aligned}
\max _{\hat{r}_{i j}^{k} \in \mathbb{R}^{+}} & \frac{1}{2} \sum_{i \in \mathcal{I}} \sum_{p_{i j}^{k} \in \mathcal{P}_{i}}\left(\mu_{i}-\lambda_{i j}\right) \hat{r}_{i j}^{k}+\sum_{l \in L^{w d}} \sum_{i \in \mathcal{I}} \sum_{p_{i j}^{k} \in \mathcal{P}_{i}} \nu_{i j}^{k, l}\left(\check{r}_{i j}^{k, l}-\hat{r}_{i j}^{k}\right) \\
& -\frac{\rho}{2} \sum_{l \in L^{w d}} \sum_{i \in \mathcal{I}} \sum_{p_{i j}^{k} \in \mathcal{P}_{i}}\left(\check{r}_{i j}^{k, l}-\hat{r}_{i j}^{k}\right)^{2} \\
\text { s.t. } & \sum_{l \in \mathcal{L}^{w l}} \frac{\sum_{i \in \mathcal{I}} \sum_{p_{i j}^{k} \in \mathcal{P}_{i}} \alpha_{i j}^{k, l} \hat{r}_{i j}^{k}}{\gamma_{n_{l} m_{l}}} \leq W,
\end{aligned}
$$

11: Update $\nu_{i j}^{k, l}$ according to:

$$
\nu_{i j}^{k, l}(t+1):=\nu_{i j}^{k, l}(t)+\rho\left(\check{r}_{i j}^{k, l}-\hat{r}_{i j}^{k}\right), \forall l \in \mathcal{L}
$$

12: $\quad t=t+1$

13: end for

14: Output the optimal bandwidth provisioning policy $\left\{r_{i j}^{k}\right\}$. 
where $L^{w d}=\left|\mathcal{L}^{w d}\right|$ is the number of wired backhaul links. Our goal is to develop a decentralized bandwidth algorithm based on ADMM that takes the form of a decomposition-coordination procedure. As the first step of ADMM, we form the augmented Lagrangian function of (5.20) as

$$
\begin{aligned}
& \mathfrak{L}_{\rho}\left(x_{l}, r_{l}^{f}, x_{l, n}\right) \\
& =\sum_{l \in L^{w d}} \frac{1}{2 L^{w d}} \sum_{i \in \mathcal{I}} \sum_{p_{i j}^{k} \in \mathcal{P}_{i}}\left(\mu_{i}-\lambda_{i j}\right) \check{r}_{i j}^{k, l}+\frac{1}{2} \sum_{i \in \mathcal{I}} \sum_{p_{i j}^{k} \in \mathcal{P}_{i}}\left(\mu_{i}-\lambda_{i j}\right) \hat{r}_{i j}^{k} \\
& +\sum_{l \in L^{w d}} \sum_{i \in \mathcal{I}} \sum_{p_{i j}^{k} \in \mathcal{P}_{i}} \nu_{i j}^{k, l}\left(\check{r}_{i j}^{k, l}-\hat{r}_{i j}^{k}\right)-\frac{\rho}{2} \sum_{l \in L^{w d}} \sum_{i \in \mathcal{I}} \sum_{p_{i j}^{k} \in \mathcal{P}_{i}}\left(\check{r}_{i j}^{k, l}-\hat{r}_{i j}^{k}\right)^{2},
\end{aligned}
$$

where $\rho>0$ is the predefined augmented Lagrangian parameter.

As the augmented Lagrangian function is separable into wireless part and wired links. ADMM algorithm can be adopted. The algorithm is summarized in Alg. 5.3. Obviously, problems (5.17) and (5.18) are all quadratic programming problems that can be solved completed decentralized across all links and SDN controller at the RAN without effort. Detailed information will be given as follows.

Following the research in [32], $\check{r}_{i j}^{k, l}$ in problem (5.17) can be updated according to the first-order derivation of the Lagrange shown as follows.

$$
\check{r}_{i j}^{k, l}=\frac{1}{2 L^{w d}}\left[\frac{\left(\mu_{i}-\lambda_{i j}\right)+\nu_{i j}^{k, l}+\rho \hat{r}_{i j}^{k}-\varpi^{l} \alpha_{i j}^{k, l}}{\rho}\right]^{+},
$$

where $\varpi^{l} \geq 0$ is the Lagrange dual variable of the capacity constraint on link $l$. Similarly, $\hat{r}_{i j}^{k}$ in problem (5.18) is calculated as follows.

$$
\hat{r}_{i j}^{k}=\frac{1}{2 \rho}\left[\begin{array}{l}
\sum_{l \in L^{w d}}\left(\nu_{i j}^{k, l}+\rho \check{r}_{i j}^{k, l}\right) \\
+\varpi \sum_{l \in L^{w l}} \frac{\alpha_{i j}^{k, l}}{\gamma_{l}}-\left(\mu_{i}-\lambda_{i j}\right)
\end{array}\right]^{+},
$$




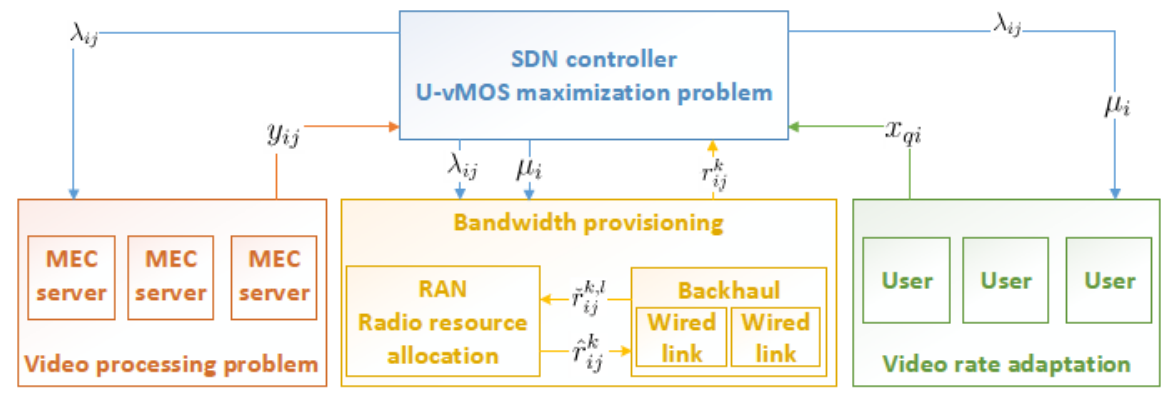

Figure 5.2: Structure and information exchange of the proposed scheme.

where $\varpi \geq 0$ is the Lagrange dual variable of the spectrum constraint on the RAN.

\subsubsection{Practical Implementation}

The overall structure and information exchange is given in Fig. 5.2. The master problem of U-vMOS maximization is solved at the SDN controller. The problem is decoupled to three parts, the computing scheduling problem solved decentralized at MEC servers, the bandwidth provisioning problem solved by the RAN and wired backhaul links, and best video resolutions selected by users. The SDN controller transfers $\lambda_{i j}$ and $\mu_{i}$ to network nodes and users to assist them to solve corresponding problem. After optimizing local variables, network elements feedback them to the SDN controller to help it to adjust $\lambda_{i j}$ and $\mu_{i}$.

As we further decouple the RAN and the wired backhaul network, information exchange between them is presented as well. Practically, the bandwidth provisioning problem may be logically solved by the SDN controller. This depends on the level of the deployment of the SDN infrastructure. If all nodes in the network are SDN-enabled, the bandwidth provisioning problem can be solved at the SDN controller. Otherwise, cooperation between SDN-enabled nodes and traditional nodes is necessary.

To illustrate the utilization of Alg. 5.2 into a SDWN, we give an instance presented 


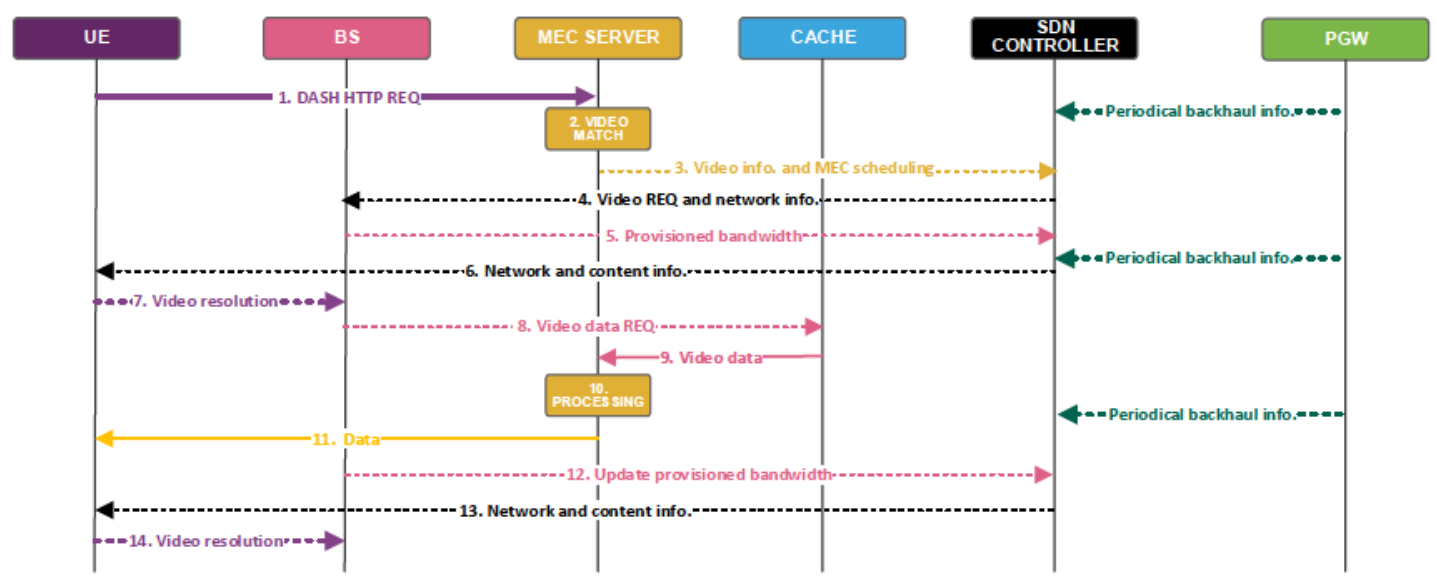

Figure 5.3: Signaling exchange in cache-enabled SDWNs.

by a signaling message exchange example in Fig. 5.3. It should be noted that video data can be retrieved from the source server and BS. In this example, we assume that the data will be retrieved from a serving BS.

As shown in Fig. 5.3, a video request (REQ) is sent by the user equipment (UE) and received by the MEC server (step 1). The MEC server seeks for matched videos in the local library (step 2). As the matched video is found, the MEC server provisions the computing resource to this potential task and sends info to the SDN controller (step 3). The SDN controller updates the network status according to feedbacks from BSs and the PGW. Video REQ and network assist information are delivered to the BS (step 4). The MEC server of the BS starts to reserve resource to compute potential task and corresponding bandwidth is provisioned by related links of the BS (step 5). After receiving this information from the BS, the SDN controller sends the network assist information to the UE to help it the select video resolution (step 6). The video resolution selection is sent to the BS and cache, then the video is processed to the desired quality level and transmitted to the UE (steps 7-11). This procedure is continue after the UE ends watching video (steps 12-14). 


\subsection{Simulation Results and Discussions}

In this section, simulation results are presented with different system configurations to demonstrate the performance of the proposed scheme.

\subsubsection{Parameters Setup}

\section{Mobile Network Configurations}

In the simulation, we consider a cellular network, consisting of one MBS, several SBSs and multiple active users, that covers a $250 \mathrm{~m}$-by-250m area. Transmission with single antenna for both transmitter and receiver is considered in this chapter. The remaining simulation parameters are summarized in Table 5.4. Values with $*$ are default values.

Table 5.4: Network parameters settings

\begin{tabular}{l|l}
\hline Network parameters & value \\
\hline \hline number of SBSs & $5-25,15^{*}$ \\
number of users & $5-25,15^{*}$ \\
frequency bandwidth $(\mathrm{MHz})$ & 20 \\
transmission power profile & SISO with maximum power; \\
& $49 \mathrm{dBm}(\mathrm{MBS}), 20 \mathrm{dBm}(\mathrm{SBS})$ \\
& pathloss:L(distance)=34+40log(distance); \\
propagation profile & lognormal shadowing: 8dB; \\
& no fast fading \\
power density of the noise & $-174 \mathrm{dBm} / \mathrm{Hz}$ \\
backhaul capacity (Mbps) & MBS to GW:100; \\
& SBS to MBS: 50 \\
\hline
\end{tabular}




\section{Video, Cache and MEC Server Configurations}

We assume that the total 1000 videos are in the video library $\mathcal{F}$. Each video $f$ can be encoded to 6 levels with constant bit rate (CBR), and has the same length of 600 seconds. Each level maps a resolution in Table 4.5.

Files in $\mathcal{F}$ have been sorted according to the popularity. We assume that the popularity of each video being requested follows a Zipf distribution with exponent 0.56 [43].

The $f$-th most popular video has a request probability of $\left(f^{-0.56}\right) /\left(\sum_{f^{\prime}=1}^{|\mathcal{F}|} f^{\prime-0.56}\right)$. The default cache capacity $S_{n}$ of a MBS is 200 video files, and default cache capacity of a SBS is 100 video files with the highest resolution, which leads the hitting rate of around $50 \%$ at MBS and $40 \%$ at SBSs. Least Frequently Used (LFU) caching policy is used at the MEC server to place/replace videos in caches, which means each BS stores the most $S_{n}$ popular video files.

The MEC computing capability of a MBS is set to $150 \mathrm{Mbps}$ that is equivalent to processing 6 videos simultaneously. Due to limitations of SBSs, the computing performance of a MEC server at SBS is only set to 50 Mbps equivalent to 2 videos.

\subsubsection{Performance Metrics and Schemes}

We evaluate the proposed system in three types of performance metrics, each of them focusing on three experiences with different network parameters, such as network load, BS density, computing resource, and cache storage capacity.

- $U$-vMOS is the objective of our proposed scheme. As pointed in [39], U-vMOS of 4.0 can be considered as a minimum requirements of the next generation mobile network.

- Ratio of HD users refers to the proportion of users that are able to select HD video (resolution higher than 1080p). 
- Average Load of MEC refers to the average working load of the MEC servers located at BS nodes.

In our evaluation, we increase the number of users and SBSs to represent the increasing network load and BS density, respectively. Meanwhile, to clear observe the effects of MEC resource, normalized computing resource and cache storage capacity are used to evaluate our proposed scheme. The term of normalized computing resource (cache storage capacity) means the ratio between the tested computing resource (cache storage capacity) to the default value.

In our simulations, we compare five different schemes shown as follows.

- $M E C C$ refers to our proposed scheme

- MECC-MBS refers to the scheme that has MEC and caches enabled at MBSs.

- Cache-only refers to the scheme that has cache enabled but MEC is not available. Therefore, videos in caches can be utilized only if the exact quality levels are matched.

- No MECC refers to the scheme that all data must be transferred from the source servers.

\subsubsection{Simulation Results}

\section{Video Quality}

In Fig. 5.4, the average U-vMOS of the overall users is presented with different network settings and MECC shemes. We can observe that the U-vMOS of the schemes with MECC is much higher than the schemes without MECC. Specifically, when the number of users increases shown in Fig. 5.4a, the average U-vMOSs decrease, as the network resources limit the performance. However, by deploying MECC, the average 


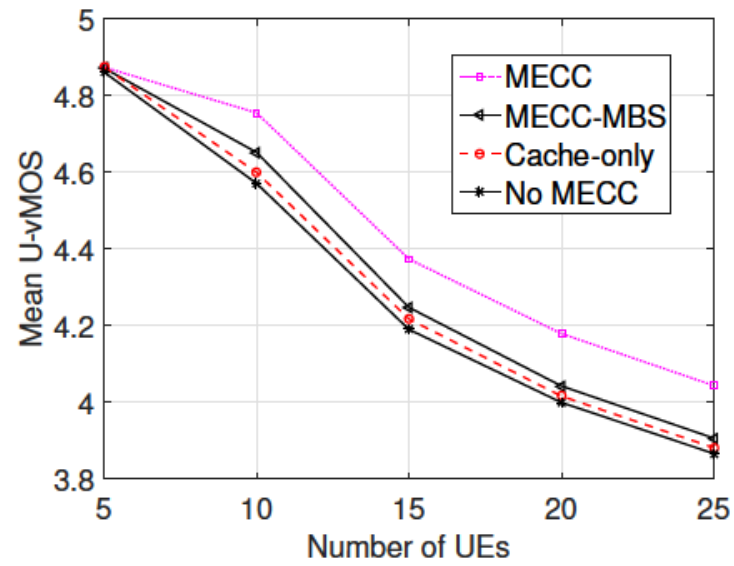

(a) Network load

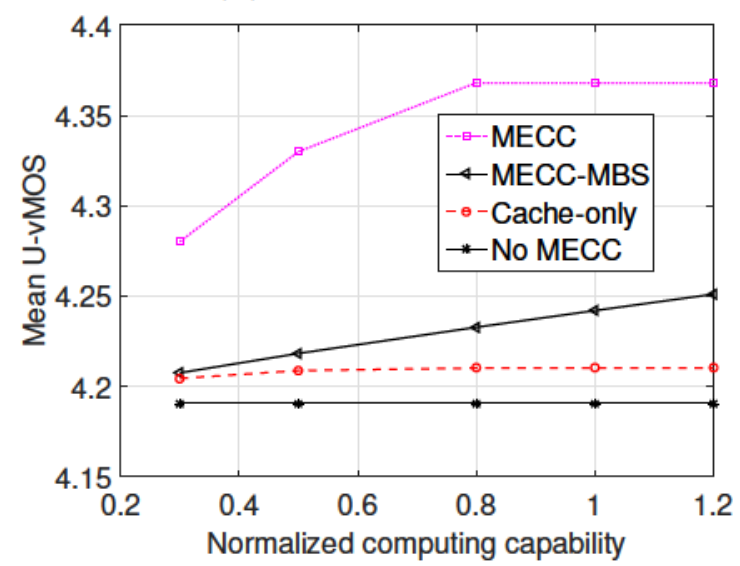

(c) Computing resource

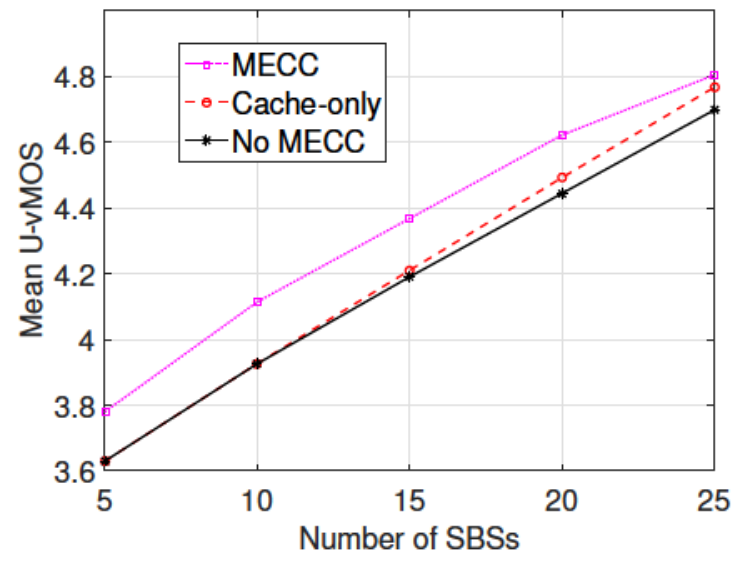

(b) BS density

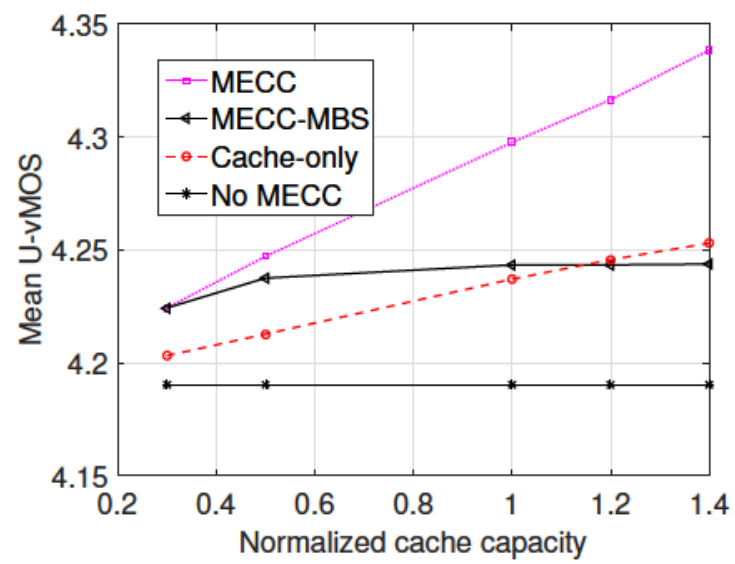

(d) Cache storage capacity

Figure 5.4: The mean U-vMoS with different network setups.

U-vMOS can reach 4 when 25 users are in the network, which means a user is able to have the resolution of $720 \mathrm{p}$ on average. To see the effect of the network density, we increase the number of SBSs in the same area as shown in Fig. 5.4b. Obviously, more SBSs lead to better performance for all cases as they can provide better coverage and channel conditions.

Figs. 5.4c and 5.4d show that increasing the capability of MEC servers and the capacity of caches can further improve the performance. It should be noted that Fig. 5.4c shows that the proposed MECC scheme becomes stable after a certain level of the capability of MEC servers, because other parameters, such as the hitting rate 


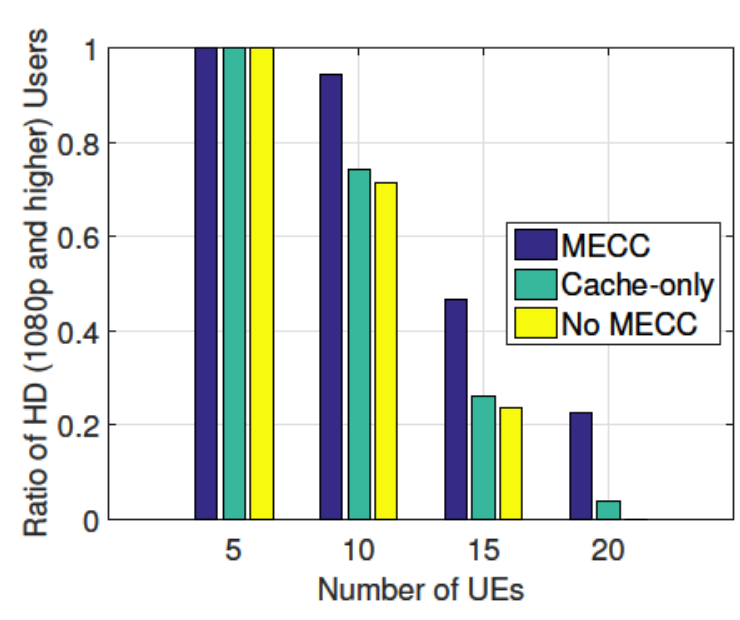

(a) Network load

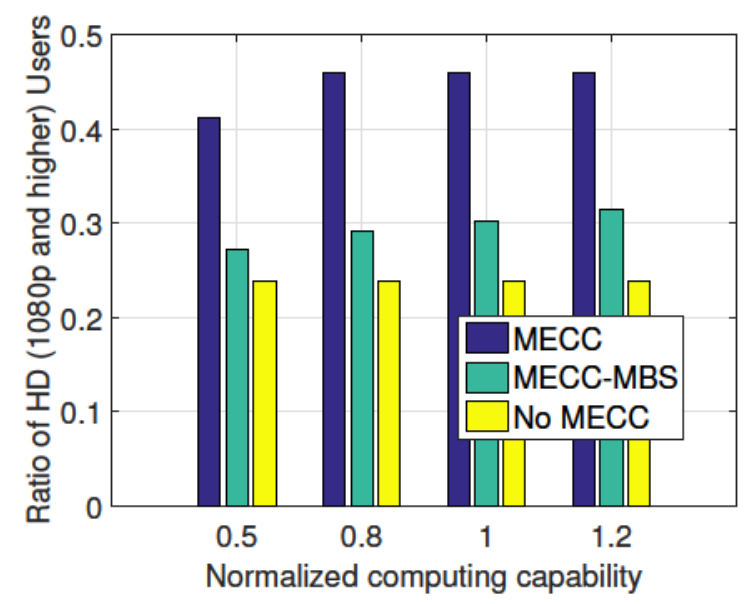

(c) Computing resource

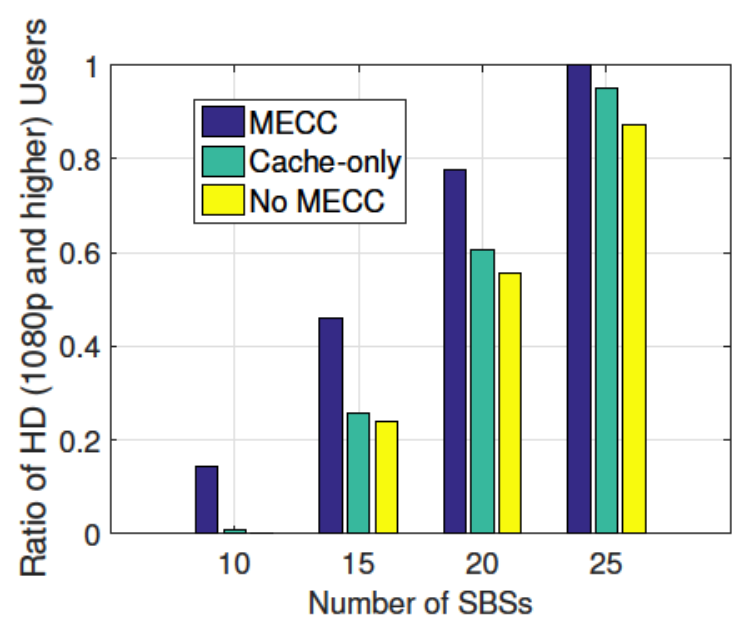

(b) BS density

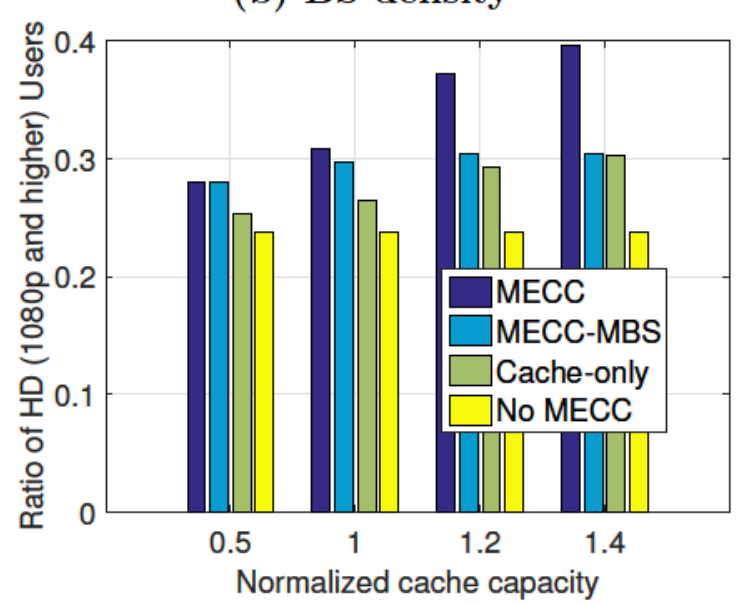

(d) Cache storage capacity

Figure 5.5: The distribution of video resolutions with different network setups.

of caches, bandwidth of both wired and wireless links, are dominated the limitation. In Fig. 5.4d, we can see that more videos stored at caches, which implicitly means higher hitting rate, give better chance to utilize the proposed MECC scheme.

In addition to the mean U-vMOS, it is also necessary to discuss the distribution of video resolutions of our proposed scheme. As shown in Fig. 5.5, overall, the trend of the distribution of video resolutions is similar to the mean U-vMOS. It can be seen that the ratio of 1080p and higher resolutions decreases with the load of the networks, 
and increases with the available network resource. The proposed scheme gets much higher ratio, up to $15 \%$, on the $1080 \mathrm{p}$ and higher resolutions compared to cache-only case and no MECC case. Note that in Figs. 5.5a and 5.5b, when the network load is high and network resource is very limited, the proposed MECC scheme still can provide around $20 \%$ and $13 \%$ of users with resolutions of 1080p and higher.

Some observations can be made from Figs. 5.4 and 5.5. Firstly, the MECC improves the performance of the network-assist video rate adaptation significantly on both average U-vMOS and HD videos. Secondly, if only MBSs can provide MEC, the gain of the proposed scheme is not as good as a traditional HetNet. Moreover, compared to traditional networks, in-network caching also can enhance the quality of video services. Furthermore, the capability of MEC servers may not always be the bottleneck of the system. Thus, in the next part, detailed tests are done on the load of MEC servers.

\section{The Load of MEC Servers}

As mentioned above, results shown in Fig. 5.6 compare the average load of MEC servers among schemes with different settings. To clear see the internal relationships, we give two companions, $M E C C$-MBS-only and MECC-SBS-only, where MEC servers are only deployed at SBSs and the MBS. We can apparently observe that the load of the MEC server at MBS is always full, which means the MEC at the MBS can be considered as the first choice of the proposed scheme because the MBS has larger coverage and the server has more capability (2 times larger than SBSs). Moreover, as shown in Fig. 5.6a, the increase of network loads leads higher computing loads as more tasks are needed to finish. Similarly, higher hitting rate also gives more opportunities to retrieve videos from network nodes, which give more tasks to servers, as shown in Fig. 5.6b. Both network settings in Figs. 5.6b and 5.6c can be treated as the 


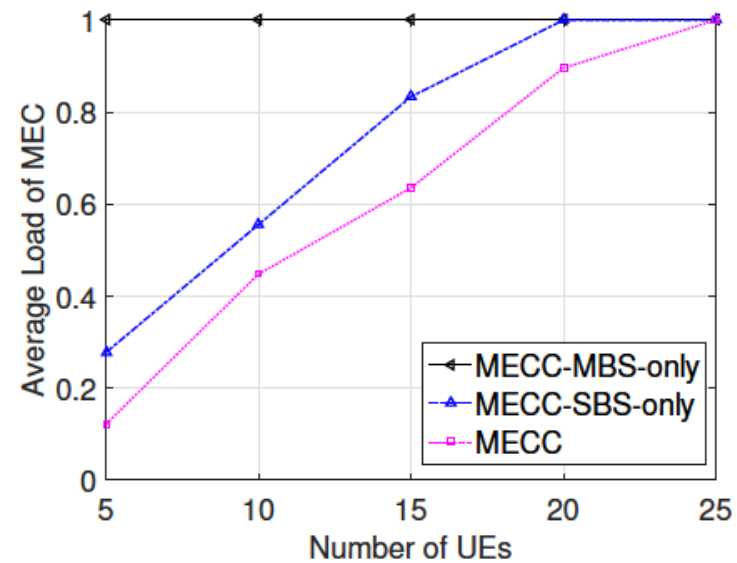

(a) Network load

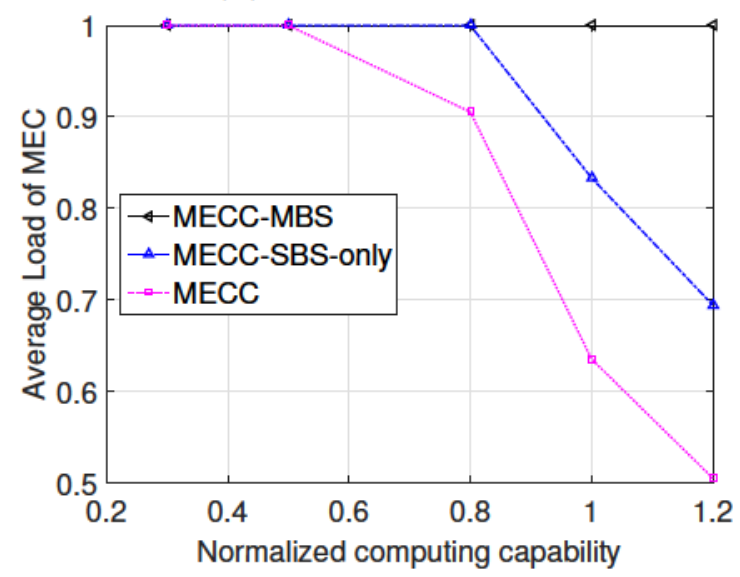

(c) Computing resource

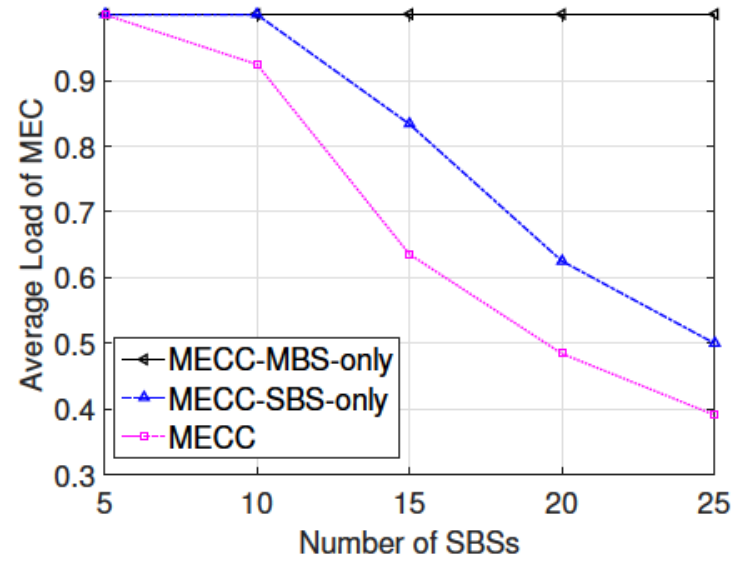

(b) BS density

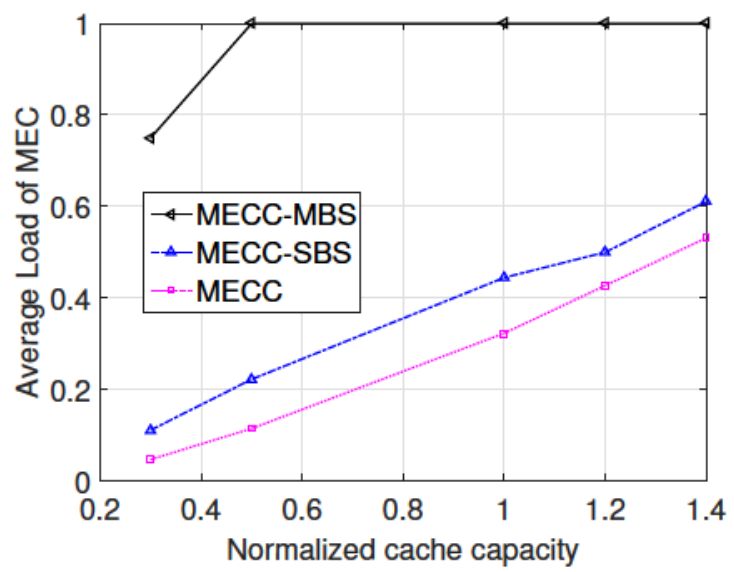

(d) Cache storage capacity

Figure 5.6: Average load of MEC servers with different network setups.

increase of available computing resources. Fig. 5.6b gives more servers as more SBSs are deployed, while Fig. 5.6c enhances each server.

\subsection{Conclusions}

In this chapter, we jointly studied the network-assisted video rate adaptation problem in a MEC-enable SDWN where in-network caching was deployed. an optimization problem was formulated with the objective of maximizing the average U-vMOS of a HetNet. Dual-decomposition method has been utilized to decouple video data rate, 
computing resource, and traffic engineering (bandwidth provisioning and path selection) so that those variable could be obtained independently. To avoid frequent exchange of network information, a decentralized algorithm based on ADMM was designed to solve the bandwidth provisioning problem. Simulation results were presented to show that our proposed scheme can significantly improve the mean U-vMOS. 


\section{Chapter 6}

\section{Conclusions and Future Works}

\subsection{Conclusions}

In this dissertation, we investigate performance improvements in software-defined and virtualized wireless networks with advanced convex optimization techniques.

- Firslty, we jointly studied wireless network virtualization and informationcentric networking in next generation cellular networks. We formulated the virtual resource allocation and in-network caching strategy as an optimization problem, which maximizes the utility of mobile virtual network operators. In addition, we developed an efficient ADMM-based distributed virtual resource allocation and in-network caching scheme. Simulation results were presented to show that the performance of backhaul alleviation can be substantially improved in the proposed scheme with in-network caching. The InPs, SPs and MVNOs can benefit from the proposed information-centric wireless network virtualization framework.

- Secondly, we jointly studied admission control and resource allocation for WNV. We proposed a two-stage VN embedding mechanism for enabling virtual network admission control and bandwidth provisioning. Firstly, we decoupled the 
admission control and physical resource leasing from physical resource allocation and bandwidth provisioning, as they are in different time scales. Then, we formulated the virtual network admission control and physical resource leasing as an robust optimization problem, which maximizes the utility of mobile virtual network provider. In addition, we took the uncertainty and fluctuation of flows rate into account and posed bandwidth provisioning as a set of chance constraints. Then, we approximated the admission control and bandwidth provisioning problems to convex problems by utilizing recent advances in robust optimization and the approximation technique. Simulation results were presented to show that the performance of physical resource reservation can be substantially improved and QoS requirements can be guaranteed.

- Moreover, we jointly studied radio resource allocation, dynamic caching and adaptive video resolution selection for cache-enabled SDWNs. We proposed a polynomial time algorithm to solve the joint problem and a signaling exchange mechanism for enabling dynamic caching in SDWNs. We took the dynamic caching and adaptive video resolution selection into account of the flow control problem in SDWNs and formulate a joint problem. Then, we transferred this problem by relaxation to a convex problem that could be solved efficiently, and an algorithm was designed. To implement our proposed algorithm into practical SDWNs, a signaling exchange diagram was designed. Simulation results were presented to show that the performance of our proposed scheme can improve the QoE in terms of delay and video quality as well as efficiency of physical resource utilization.

- At last, we jointly studied the network-assisted video rate adaptation problem in a MEC-enable SDWN where in-network caching was deployed. an optimization problem was formulated with the objective of maximizing the average U-vMOS 
of a HetNet. Dual-decomposition method has been utilized to decouple video data rate, computing resource, and traffic engineering (bandwidth provisioning and path selection) so that those variable could be obtained independently. To avoid frequent exchange of network information, a decentralized algorithm based on ADMM was designed to solve the bandwidth provisioning problem. Simulation results were presented to show that our proposed scheme can significantly improve the mean U-vMOS.

\subsection{Future Works}

A number of interesting research problems rise during the course of the investigations reported in this dissertation.

- Energy-efficient techniques are significant topics in next generation mobile networks, as energy consumption has become a primary concern in the design and operation of wireless communication systems [115]. As MEC and in-network caching are key components of 5G, energy efficiency has become a main concern for the design of those mechanisms. Energy-efficient MEC has been investigated from the aspect of computation offloading $[14,116,117]$. In the line of caching, some studies (e.g., [118-120]) focus on how to energy-efficiently utilize the cache and network resource to place contents. However, energy-efficient resource allocation that considers the utilization of both MEC and in-network caching has been largely ignored in the existing research.

- For the schemes presented, we assume that wireless nodes can use full power and advanced power allocation schemes are not considered. That means the potential benefit of power control is not fully investigated. Indeed, in wireless networks, the power control problem has been investigated for quite a long 
time. However, there are few studies consider power allocation schemes with virtualization, caching, MEC and SDWNs. Moreover, massive multiple-inputmultiple-output (mMIMO) has been proposed to be the key enabler of 5G. It is attractive to deploy advanced power allocation techniques and mMIMO in our proposed schemes.

- Unlike services provided by current mobile networks only focus on voice and data, the services supported by $5 \mathrm{G}$ can range from high data rate services (e.g., VR) to ultra-reliable low latency communication (e.g., vehicle communication) to mass machine type communication (e.g., IoT). In this dissertation, only traditional services that provision high data rate are considered. It is necessary to extend our works to other types of networks. Obviously, those lines of extension will not be straightforward, which may lead to significantly changes on the network architecture.

- The last promising direction is to explore the interaction of the advanced machine learning with wireless networks or computer networks in general. One question related to wireless communications is how to utilize machine learning to investigage the traffic in wireless networks. Furthermore, another promisiong application of machine learning in wireless network in how to design the infrastructure to support machine learning. 


\section{List of References}

[1] Cisco, "Cisco visual networking index: Global mobile data traffic forecast update 2015-2020," tech. rep., Feb. 2016.

[2] X. Wang, M. Chen, T. Taleb, A. Ksentini, and V. Leung, "Cache in the air: exploiting content caching and delivery techniques for 5G systems," IEEE Commun. Mag., vol. 52, pp. 131-139, Feb. 2014.

[3] X. Wang, P. Krishnamurthy, and D. Tipper, "Wireless network virtualization," in Proc. Int'l Conf. on Computing, Networking and Commun. (ICNC), (San Diego, CA), Jan. 2013.

[4] C. Liang and F. R. Yu, "Wireless network virtualization: A survey, some research issues and challenges," IEEE Commun. Surveys \& Tutorials, vol. 17, pp. 358-380, Firstquarter 2015.

[5] C. J. Bernardos, A. De La Oliva, P. Serrano, A. Banchs, L. M. Contreras, H. Jin, and J. C. Zúñiga, "An architecture for software defined wireless networking," IEEE Wireless Comm., vol. 21, pp. 52-61, June 2014.

[6] Y. C. Hu, M. Patel, D. Sabella, N. Sprecher, and V. Young, "White paper: Mobile edge computing: A key technology towards 5G," tech. rep., ETSI, Dec. 2015.

[7] B. Ahlgren, C. Dannewitz, C. Imbrenda, D. Kutscher, and B. Ohlman, "A survey of information-centric networking," IEEE Commun. Mag., vol. 50, no. 7, pp. 26-36, Jul. 2012.

[8] J. Choi, J. Han, E. Cho, T. T. Kwon, and Y. Choi, "A survey on contentoriented networking for efficient content delivery," IEEE Commun. Mag., vol. 49, no. 3, pp. 121-27, Mar. 2011. 
[9] S. Paul, J. Pan, and R. Jain, "Architectures for the future networks and the next generation internet: a survey," Comput. Commun., vol. 34, no. 1, pp. 2-42, Jan. 2011.

[10] "FP7 PURSUIT Project."

[11] "NSF Named Data Networking Project."

[12] G. Paschos, E. Baştuğ, I. Land, G. Caire, and M. Debbah, "Wireless caching: Technical misconceptions and business barriers," IEEE Comm. Mag., vol. 54, pp. 16-22, Aug. 2016.

[13] D. Liu, B. Chen, C. Yang, and A. F. Molisch, "Caching at the wireless edge: design aspects, challenges, and future directions," IEEE Comm. Mag., vol. 54, pp. 22-28, Sept. 2016.

[14] X. Chen, L. Jiao, W. Li, and X. Fu, "Efficient multi-user computation offloading for mobile-edge cloud computing," vol. 24, pp. 2795-2808, Oct. 2016.

[15] M. Patel, B. Naughton, C. Chan, N. Sprecher, S. Abeta, A. Neal, et al., "Mobileedge computing introductory technical white paper," tech. rep., ETSI, Sept. 2014.

[16] N. Chowdhury and R. Boutaba, "Network virtualization: state of the art and research challenges," IEEE Commun. Mag., vol. 47, pp. 20-26, July 2009.

[17] G. ETSI, "Network functions virtualisation (NFV): Use cases," Tech. Rep. 2013001, Mar. 2013.

[18] R. Mijumbi, J. Serrat, J.-L. Gorricho, N. Bouten, F. De Turck, and R. Boutaba, "Network function virtualization: State-of-the-art and research challenges," IEEE Commun. Surveys \& Tutorials, vol. 18, pp. 236-262, Firstquarter 2016.

[19] A. Fischer, J. Botero, M. Beck, H. De Meer, and X. Hesselbach, "Virtual network embedding: A survey," IEEE Commun. Surveys \& Tutorials, vol. to be published, 2013.

[20] T. K. Forde, I. Macaluso, and L. E. Doyle, "Exclusive sharing \& virtualization of the cellular network," in Proc. IEEE Symp. on New Frontiers in Dynamic Spectrum Access Net. (DySPAN), (Aachen), May 2011.

[21] H. Wen, P. K. Tiwary, and T. Le-Ngoc, Wireless Virtualization. Springer, 2013. 
[22] X. Costa-Perez, J. Swetina, G. Tao, R. Mahindra, and S. Rangarajan, "Radio access network virtualization for future mobile carrier networks," IEEE Commun. Mag., vol. 51, July 2013.

[23] R. Kokku, R. Mahindra, H. Zhang, and S. Rangarajan, "Nvs: a substrate for virtualizing wireless resources in cellular networks," IEEE/ACM Trans. Netw., vol. 20, pp. 1333-1346, Oct. 2012.

[24] C. Liang and F. R. Yu, "Wireless virtualization for next generation mobile cellular networks," IEEE Wireless Comm., vol. 22, pp. 61-69, Mar. 2015.

[25] H. Wen, P. K. Tiwary, and L.-N. Tho, "Current trends and perspectives in wireless virtualization," in Proc. Int'l Conference on Selected Topics in Mobile 8 Wireless Networking (MoWNeT), (Montreal, Canada), Aug. 2013.

[26] ONF, "Onf white paper: Software-defined networking: The new norm for networks," tech. rep., ONF, 2012.

[27] B. A. Nunes, M. Mendonca, X.-N. Nguyen, K. Obraczka, and T. Turletti, "A survey of software-defined networking: past, present, and future of programmable networks," IEEE Commun. Surveys $\&$ Tutorials, vol. 16, pp. 16171634, Aug. 2014.

[28] T. Chen, M. Matinmikko, X. Chen, X. Zhou, and P. Ahokangas, "Software defined mobile networks: concept, survey, and research directions," IEEE Commun. Mag., vol. 53, pp. 126-133, Nov. 2015.

[29] S. Sun, L. Gong, B. Rong, and K. Lu, "An intelligent SDN framework for 5G heterogeneous networks," IEEE Commun. Mag., vol. 53, pp. 142-147, Nov. 2015 .

[30] C. Yang, Z. Chen, B. Xia, and J. Wang, "When ICN meets C-RAN for HetNets: an SDN approach," IEEE Commun. Mag., vol. 53, pp. 118-125, Nov. 2015.

[31] F. Granelli, A. A. Gebremariam, M. Usman, F. Cugini, V. Stamati, M. Alitska, and P. Chatzimisios, "Software defined and virtualized wireless access in future wireless networks: scenarios and standards," IEEE Comm. Mag., vol. 53, pp. 26-34, June 2015.

[32] W.-C. Liao, M. Hong, H. Farmanbar, X. Li, Z.-Q. Luo, and H. Zhang, "Min flow rate maximization for software defined radio access networks," IEEE J. Sel. Areas Commun., vol. 32, pp. 1282-1294, June 2014. 
[33] A. Mendiola, J. Astorga, E. Jacob, and M. Higuero, "A survey on the contributions of software-defined networking to traffic engineering," IEEE Commun. Surveys 8 Tutorials, vol. PP, no. 99, pp. 1-1, 2016.

[34] H. Farmanbar and H. Zhang, "Traffic engineering for software-defined radio access networks," in Proc. IEEE Netw. Operations 8 Management Sym. (NOMS), pp. 1-7, May 2014.

[35] C. Liang, F. R. Yu, and X. Zhang, "Information-centric network function virtualization over 5G mobile wireless networks," Network, IEEE, vol. 29, pp. 68-74, Mar. 2015.

[36] Y. Wen, X. Zhu, J. J. P. C. Rodrigues, and C. W. Chen, "Cloud mobile media: Reflections and outlook," IEEE Trans. Multimedia, vol. 16, pp. 885-902, June 2014.

[37] Z. He, S. Mao, and S. Kompella, "Quality of experience driven multi-user video streaming in cellular cognitive radio networks with single channel access," IEEE Trans. Multimedia, vol. 18, pp. 1401-1413, July 2016.

[38] D. Schoolar, "Whitepaper: Mobile video requires performance and measurement standards," 2015.

[39] Huawei Technologies Co.,Ltd, "Whitepaper: 4.5G, opening Giga mobile world, empowering vertical markets," 2016.

[40] H. A. Pedersen and S. Dey, "Enhancing mobile video capacity and quality using rate adaptation, RAN caching and processing," ACM/IEEE Trans. Networking, vol. 24, pp. 996-1010, May 2016.

[41] Y. Chen, K. Wu, and Q. Zhang, "From QoS to QoE: a tutorial on video quality assessment," IEEE Commun. Surveys \& Tutorials, vol. 17, pp. 1126-1165, Oct. 2015.

[42] N.-D. Dao, H. Zhang, H. Farmanbar, X. Li, and A. Callard, "Handling realtime video traffic in software-defined radio access networks," in Proc. IEEE ICC workshops, pp. 191-196, June 2015.

[43] N. Golrezaei, K. Shanmugam, A. G. Dimakis, A. F. Molisch, and G. Caire, "Femtocaching: Wireless video content delivery through distributed caching helpers," in Proc. IEEE INFOCOM, pp. 1107-1115, Mar. 2012. 
[44] T. X. Tran, P. Pandey, A. Hajisami, and D. Pompili, "Collaborative multibitrate video caching and processing in mobile-edge computing networks," arXiv preprint arXiv:1612.01436, 2016.

[45] M. S. ElBamby, M. Bennis, W. Saad, and M. Latva-aho, "Content-aware user clustering and caching in wireless small cell networks," in Int. Sym. onWireless Commun. Sys. (ISWCS), pp. 945-949, Aug. 2014.

[46] A. Liu and V. K. Lau, "Mixed-timescale precoding and cache control in cached mimo interference network," IEEE Trans. Signal Processing, vol. 61, pp. 63206332, Sept. 2013.

[47] B. Dai and W. Yu, "Sparse beamforming and user-centric clustering for downlink cloud radio access network," IEEE Access, vol. 2, pp. 1326-1339, Nov. 2014.

[48] A. Abboud, E. Bastug, K. Hamidouche, and M. Debbah, "Distributed caching in 5G networks: An alternating direction method of multipliers approach," in Proc. IEEE Workshop on Signal Processing Advances in Wireless Commun. (SPAWC), pp. 171-175, July 2015.

[49] H. Li, Z. Wang, and D. Hu, "Joint wireless and backhaul load balancing in cooperative caches enabled small-cell networks," in Proc. IEEE Symp. Personal, Indoor \& Mobile Radio Commun. (PIMRC), pp. 1889-1894, Sept. 2015.

[50] K. Poularakis, G. Iosifidis, and L. Tassiulas, "Joint caching and base station activation for green heterogeneous cellular networks," in Proc. IEEE Int. Conf. Commun. (ICC), pp. 3364-3369, June 2015.

[51] V. A. Siris, X. Vasilakos, and G. Polyzos, "Efficient proactive caching for supporting seamless mobility," in Proc. IEEE 15th Int. Sym. on a World of Wireless, Mobile and Multimedia Netw. (WoWMoM), pp. 1-6, June 2014.

[52] S. Singh, O. Oyman, A. Papathanassiou, D. Chatterjee, and J. G. Andrews, "Video capacity and QoE enhancements over LTE," in Proc. IEEE Int. Conf. Commun. (ICC), pp. 7071-7076, June 2012.

[53] D. De Vleeschauwer, H. Viswanathan, A. Beck, S. Benno, G. Li, and R. Miller, "Optimization of HTTP adaptive streaming over mobile cellular networks," in Proc. IEEE INFOCOM, pp. 898-997, Apr. 2013. 
[54] A. Argyriou, D. Kosmanos, and L. Tassiulas, "Joint time-domain resource partitioning, rate allocation, and video quality adaptation in heterogeneous cellular networks," IEEE Trans. Multimedia, vol. 17, pp. 736-745, May 2015.

[55] A. El Essaili, D. Schroeder, E. Steinbach, D. Staehle, and M. Shehada, "QoEbased traffic and resource management for adaptive HTTP video delivery in LTE," IEEE Trans. Circ. Sys. Video Tech., vol. 25, pp. 988-1001, June 2015.

[56] V. Joseph, S. Borst, and M. I. Reiman, "Optimal rate allocation for video streaming in wireless networks with user dynamics," ACM/IEEE Trans. Networking, vol. 24, pp. 820-835, Mar. 2016.

[57] M. Seufert, S. Egger, M. Slanina, T. Zinner, T. Hoßfeld, and P. Tran-Gia, "A survey on quality of experience of HTTP adaptive streaming," IEEE Commun. Surveys $\&$ Tutorials, vol. 17, pp. 469-492, Sept. 2015.

[58] H. Ahlehagh and S. Dey, "Video-aware scheduling and caching in the radio access network," IEEE/ACM Trans. Netw., vol. 22, pp. 1444-1462, Oct. 2014.

[59] J. Zhu, J. He, H. Zhou, and B. Zhao, "Epcache: In-network video caching for LTE core networks," in Proc. Int'l Conf. Wireless Communications 83 Signal Processing (WCSP), pp. 1-6, Oct. 2013.

[60] A. Liu and V. K. Lau, "Exploiting base station caching in mimo cellular networks: Opportunistic cooperation for video streaming," IEEE Trans. Signal Processing, vol. 63, pp. 57-69, Jan. 2015.

[61] R. Yu, S. Qin, M. Bennis, X. Chen, G. Feng, Z. Han, and G. Xue, "Enhancing software-defined RAN with collaborative caching and scalable video coding," in Proc. IEEE Int. Conf. Commun. (ICC), pp. 1-6, May 2016.

[62] C. Ge, N. Wang, S. Skillman, G. Foster, and Y. Cao, "QoE-driven dash video caching and adaptation at 5G mobile edge," in Proc. ACM Conference on Information-Centric Networking, pp. 237-242, Sept. 2016.

[63] L. Peterson, T. Anderson, D. Blumenthal, D. Casey, D. Clark, D. Estrin, J. Evans, D. Raychaudhuri, M. Reiter, J. Rexford, et al., "GENI design principles," IEEE Computer, vol. 39, pp. 102-105, Sept. 2006.

[64] L. Sarakis, T. Zahariadis, H.-C. Leligou, and M. Dohler, "A framework for service provisioning in virtual sensor networks," EURASIP J. on Wireless Commun. \& Netw., vol. 2012, no. 1, pp. 1-19, 2012. 
[65] L. Xia, S. Kumar, X. Yang, P. Gopalakrishnan, Y. Liu, S. Schoenberg, and $\mathrm{X}$. Guo, "Virtual wifi: bring virtualization from wired to wireless," $A C M S I G$ PLAN Not., vol. 46, pp. 181-192, Mar. 2011.

[66] K. Pentikousis, W. Yan, and H. Weihua, "Mobileflow: Toward software-defined mobile networks," IEEE Commun. Mag., vol. 51, pp. 44 - 53, July 2013.

[67] Y. Zaki, LTE Optimization and Mobile Network Virtualization. Springer, 2012.

[68] G. Liu, F. R. Yu, H. Ji, and V. Leung, "Distributed resource allocation in fullduplex relaying networks with wireless virtualization," in Proc. IEEE Globecom, pp. 4959-4964, Dec. 2014.

[69] M. I. Kamel, L. B. Le, and A. Girard, "Lte wireless network virtualization: Dynamic slicing via flexible scheduling," in Proc. IEEE Veh. Tech. Conf. (VTC) - Spring, pp. 1-5, 2014.

[70] M. Yang, Y. Li, J. Liu, D. Jin, J. Yuan, and L. Zeng, "Opportunistic spectrum sharing for wireless virtualization," in Proc. IEEE Wireless Commun. \& Networking Conf. (WCNC), pp. 1803-1808, 2014.

[71] S. Agarwal, M. Kodialam, and T. Lakshman, "Traffic engineering in software defined networks," in Proc. IEEE INFOCOM, pp. 2211-2219, Apr. 2013.

[72] L.-H. Huang, H.-C. Hsu, S.-H. Shen, D.-N. Yang, and W.-T. Chen, "Multicast traffic engineering for software-defined networks," in Proc. IEEE INFOCOM, pp. 1-9, Apr. 2016.

[73] H. Huang, S. Guo, W. Liang, K. Li, B. Ye, and W. Zhuang, "Near-optimal routing protection for in-band software-defined heterogeneous networks," IEEE J. Sel. Areas Commun., vol. 34, pp. 2918-2934, Nov. 2016.

[74] S. Boyd, N. Parikh, E. Chu, B. Peleato, and J. Eckstein, "Distributed optimization and statistical learning via the alternating direction method of multipliers," Foundations and Trends in Machine Learning, vol. 3, pp. 1-122, Jan. 2011.

[75] 3GPP, "Evolved Universal Terrestrial Radio Access (E-UTRA); Physical channels and modulation," TS 23.211 V12.2.0, 3rd Generation Partnership Project (3GPP), Jul. 2014.

[76] D. W. K. Ng, E. S. Lo, and R. Schober, "Energy-efficient resource allocation in multi-cell ofdma systems with limited backhaul capacity," IEEE Trans. Wireless Commun., vol. 11, pp. 3618-3631, Sept. 2012. 
[77] P. Blasco and D. Gunduz, "Learning-based optimization of cache content in a small cell base station," arXiv preprint arXiv:1402.3247, Feb. 2014.

[78] M. Fiore, F. Mininni, C. Casetti, and C. Chiasserini, "To cache or not to cache?," in Proc. IEEE INFOCOM, pp. 235-243, Apr. 2009.

[79] C. Fricker, P. Robert, J. Roberts, and N. Sbihi, "Impact of traffic mix on caching performance in a content-centric network," in Proc. IEEE INFOCOM, pp. 310-315, Mar. 2012.

[80] D. Bethanabhotla, O. Y. Bursalioglu, H. C. Papadopoulos, and G. Caire, "User association and load balancing for cellular massive mimo," in Proc. Information Theory and Applications Workshop (ITA), pp. 1-10, Feb. 2014.

[81] Q. Ye, B. Rong, Y. Chen, M. Al-Shalash, C. Caramanis, and J. G. Andrews, "User association for load balancing in heterogeneous cellular networks," IEEE Trans. Wireless Commun., vol. 12, pp. 2706-2716, June 2013.

[82] D. Fooladivanda and C. Rosenberg, "Joint resource allocation and user association for heterogeneous wireless cellular networks," IEEE Trans. Wireless Commun., vol. 12, pp. 248-257, Jan. 2013.

[83] S. Gortzen and A. Schmeink, "Optimality of dual methods for discrete multiuser multicarrier resource allocation problems," IEEE Trans. Wireless Commun., vol. 11, pp. 3810-3817, Oct. 2012.

[84] S. Boyd and L. Vandenberghe, Convex Optimization. Cambridge university press, 2009.

[85] J. G. Andrews, H. Claussen, M. Dohler, S. Rangan, and M. C. Reed, "Femtocells: Past, present, and future," IEEE J. Sel. Areas Commun., vol. 30, pp. 497508, Apr. 2012.

[86] 3GPP, "Evolved Universal Terrestrial Radio Access (E-UTRA); Further advancements for E-UTRA physical layer aspects," TR 36.814 V9.0.0, 3rd Generation Partnership Project (3GPP), Mar. 2010.

[87] H. J. Kushner and P. A. Whiting, "Convergence of proportional-fair sharing algorithms under general conditions," IEEE Trans. Wireless Commun., vol. 3, pp. 1250-1259, Apr. 2004.

[88] Y. Zaki, L. Zhao, C. Goerg, and A. Timm-Giel, "LTE mobile network virtualization," Mobile Net. \& Applications, vol. 16, pp. 424-432, Aug. 2011. 
[89] Y. Fang and Y. Zhang, "Call admission control schemes and performance analysis in wireless mobile networks," IEEE Trans. Veh. Technol., vol. 51, pp. 371382, Mar. 2002.

[90] F. Yu, V. Krishnamurthy, and V. C. M. Leung, "Cross-layer optimal connection admission control for variable bit rate multimedia traffic in packet wireless CDMA networks," IEEE Trans. Signal Proc., vol. 54, pp. 542-555, Feb. 2006.

[91] D. Bertsimas, D. B. Brown, and C. Caramanis, "Theory and applications of robust optimization," SIAM review, vol. 53, no. 3, pp. 464-501, 2011.

[92] A. Ben-Tal and A. Nemirovski, "Robust solutions of linear programming problems contaminated with uncertain data," Mathematical programming, vol. 88, no. 3, pp. 411-424, 2000.

[93] B. K. Chalise and L. Vandendorpe, "Optimization of mimo relays for multipoint-to-multipoint communications: Nonrobust and robust designs," Signal Processing, IEEE Transactions on, vol. 58, no. 12, pp. 6355-6368, 2010.

[94] M. Hasan, E. Hossain, and D. I. Kim, "Resource allocation under channel uncertainties for relay-aided device-to-device communication underlaying lte-a cellular networks," IEEE Trans. Wireless Commun., vol. 13, no. 4, 2014.

[95] L. Wang, M. Sheng, Y. Zhang, X. Wang, and C. Xu, "Robust energy efficiency maximization in cognitive radio networks: The worst-case optimization approach," IEEE Trans. Commun., vol. 63, pp. 51-65, Jan 2015.

[96] S. Yun and C. Caramanis, "System-level optimization in wireless networks: managing interference and uncertainty via robust optimization," ACM/IEEE Trans. Networking, vol. 20, pp. 339-352, Apr. 2012.

[97] N. Y. Soltani, S.-J. Kim, and G. B. Giannakis, "Chance-constrained optimization of ofdma cognitive radio uplinks," IEEE Trans. Wireless Commun., vol. 12, pp. 1098-1107, Mar. 2013.

[98] A. Ben-Tal and A. Nemirovski, "Selected topics in robust convex optimization," Mathematical Programming, vol. 112, pp. 125-158, Mar. 2008.

[99] A. Nemirovski and A. Shapiro, "Convex approximations of chance constrained programs," SIAM Journal on Optimization, vol. 17, pp. 969-996, Nov. 2006.

[100] M. Grant and S. Boyd, "CVX: Matlab software for disciplined convex programming, version 2.1," Mar. 2014. 
[101] G. Liu, F. R. Yu, H. Ji, and V. C. Leung, "Energy-efficient resource allocation in cellular networks with shared full-duplex relaying," IEEE Trans. Veh. Tech., vol. 64, pp. 3711-3724, Aug. 2015.

[102] J. Li, Y. Chen, Z. Lin, W. Chen, B. Vucetic, and L. Hanzo, "Distributed caching for data dissemination in the downlink of heterogeneous networks," IEEE Trans. Commun., vol. 63, pp. 3553-3568, Oct. 2015.

[103] M. Tao, E. Chen, H. Zhou, and W. Yu, "Content-centric sparse multicast beamforming for cache-enabled cloud RAN," IEEE Trans. Wireless Commun., vol. 15, pp. pp-6118, Sept. 2016.

[104] C. Liang, F. Yu, H. Yao, and Z. Han, "Virtual resource allocation in information-centric wireless networks with virtualization," IEEE Trans. Veh. Tech., vol. 65, pp. 9902-9914, Dec. 2016.

[105] D. Bethanabhotla, O. Y. Bursalioglu, H. C. Papadopoulos, and G. Caire, "Optimal user-cell association for massive mimo wireless networks," IEEE Trans. Wireless Commun., vol. 15, pp. 1835-1850, Mar. 2016.

[106] J. Qiao, Y. He, and X. S. Shen, "Proactive caching for mobile video streaming in millimeter wave 5G networks," IEEE Trans. Wireless Commun., vol. 15, pp. 7187-7198, Oct. 2016.

[107] Y. Zhang, "User mobility from the view of cellular data networks," in Proc. IEEE INFOCOM, pp. 1348-1356, May 2014.

[108] G. Li and H. Liu, "Downlink radio resource allocation for multi-cell OFDMA system," IEEE Trans. Wireless Commun., vol. 5, pp. 3451-3459, Dec. 2006.

[109] S. Boyd, L. Xiao, A. Mutapcic, and J. Mattingley, "Notes on decomposition methods," Notes for EE364B, Stanford University, pp. 1-36, 2007.

[110] P. Si, H. Yue, Y. Zhang, and Y. Fang, "Spectrum management for proactive video caching in information-centric cognitive radio networks," IEEE J. Sel. Areas Commun., vol. 34, pp. 2247-2259, Aug. 2016.

[111] J. Eckstein, "Augmented lagrangian and alternating direction methods for convex optimization: A tutorial and some illustrative computational results," RUTCOR Research Reports, vol. 32, 2012. 
[112] D. P. Palomar and M. Chiang, "A tutorial on decomposition methods for network utility maximization," IEEE J. Sel. Areas Commun., vol. 24, pp. 14391451, Aug. 2006.

[113] Q.-D. Vu, L.-N. Tran, M. Juntti, and E.-K. Hong, "Energy-efficient bandwidth and power allocation for multi-homing networks," IEEE Trans. Signal Proc., vol. 63, pp. 1684-1699, June 2015.

[114] H. Farmanbar and H. Zhang, "Cross-layer traffic engineering for softwaredefined radio access networks," in Proc. IEEE Int. Conf. Commun. (ICC), pp. 3411-3416, June 2015.

[115] S. Buzzi, I. Chih-Lin, T. E. Klein, H. V. Poor, C. Yang, and A. Zappone, "A survey of energy-efficient techniques for 5G networks and challenges ahead," IEEE J. Sel. Areas Commun., vol. 34, pp. 697-709, Apr. 2016.

[116] Y. Mao, J. Zhang, and K. B. Letaief, "Dynamic computation offloading for mobile-edge computing with energy harvesting devices," IEEE J. Sel. Areas Commun., vol. 34, pp. 3590-3605, Dec. 2016.

[117] C. You, K. Huang, H. Chae, and B.-H. Kim, "Energy-efficient resource allocation for mobile-edge computation offloading," IEEE Trans. Wireless Commun., vol. PP, no. 99, pp. 1-1, 2016.

[118] J. Llorca, A. M. Tulino, M. Varvello, J. Esteban, and D. Perino, "Energy efficient dynamic content distribution," IEEE J. Sel. Areas Commun., vol. 33, pp. 2826-2836, Dec. 2015.

[119] D. Liu and C. Yang, "Energy efficiency of downlink networks with caching at base stations," IEEE J. Sel. Areas Commun., vol. 34, pp. 907-922, Apr. 2016.

[120] F. Gabry, V. Bioglio, and I. Land, "On energy-efficient edge caching in heterogeneous networks," IEEE J. Sel. Areas Commun., vol. 34, pp. 3288-3298, Dec. 2016.

[121] M. Leinonen, M. Codreanu, and M. Juntti, "Distributed joint resource and routing optimization in wireless sensor networks via alternating direction method of multipliers," IEEE Trans. Wireless Commun., vol. 12, pp. 5454-5467, Nov. 2013. 


\section{Appendix A}

\section{Introduction to ADMM with Consensus}

\section{Constraint}

$\operatorname{ADMM}[74,111]$ is a simple but powerful algorithm that is well suited to distributed convex optimization. One of the important properties of ADMM is its quick convergence to a modest accuracy of the optimal solution. It has been successfully used in many cases, such as in statistical learning problems, engineering design, multi-period portfolio optimization, time series analysis, network flow, and scheduling [74,111,121]. It takes the form of a decomposition-coordination procedure, in which the solutions to small local subproblems are coordinated to find a solution to a large global problem. Furthermore, ADMM can be viewed as an attempt to blend the benefits of dual decomposition and augmented Lagrangian methods for constrained optimization [74], [111]. Generally, ADMM is able to solve

$$
\begin{aligned}
& \min _{\mathbf{x}, \mathbf{z}} f(\mathbf{x})+g(\mathbf{z}) \\
& \text { s.t. } \quad \mathbf{A} \mathbf{x}+\mathbf{B z}=\mathbf{c},
\end{aligned}
$$

where $\mathbf{x} \in \mathbb{R}^{q \times 1}, \mathbf{z} \in \mathbb{R}^{r \times 1}, \mathbf{A} \in \mathbb{R}^{p \times q}, \mathbf{B} \in \mathbb{R}^{p \times r}$ and $\mathbf{c} \in \mathbb{R}^{p \times 1}$. There are two basic forms for the ADMM algorithm, namely the unscaled form and the scaled form. In 
the unscaled form, the augmented Lagrangian is given as follows.

$$
\begin{aligned}
L_{\rho}(\mathbf{x}, \mathbf{y}, \mathbf{z}) & =f(\mathbf{x})+g(\mathbf{z})+\mathbf{y}^{T}(\mathbf{A} \mathbf{x}+\mathbf{B z}-\mathbf{c}) \\
& +(\rho / 2)\|\mathbf{A} \mathbf{x}+\mathbf{B} \mathbf{z}-\mathbf{c}\|_{2}^{2},
\end{aligned}
$$

where $\mathbf{y} \in \mathbb{R}^{p \times 1}$ is the dual variable vector, $\rho>0$ is the predefined augmented Lagrangian parameter and $\|\cdot\|_{2}$ is an Euclidean norm operator. Accordingly, the unscaled ADMM algorithm consists of the following iterations:

$$
\begin{array}{r}
\mathbf{x}^{t+1}:=\arg \min _{\mathbf{x}} L_{\rho}\left(\mathbf{x}, \mathbf{y}^{t}, \mathbf{z}^{t}\right), \\
\mathbf{z}^{t+1}:=\arg \min _{\mathbf{z}} L_{\rho}\left(\mathbf{x}^{t+1}, \mathbf{y}^{t}, \mathbf{z}\right), \\
\mathbf{y}^{t+1}:=\mathbf{y}^{t}+\rho\left(\mathbf{A x}^{t+1}+\mathbf{B} \mathbf{z}^{t+1}-\mathbf{c}\right),
\end{array}
$$

where $t$ is the iteration index.

Let us consider another problem with local variables $x_{i} \in \mathbb{R}^{n}$ and a common global variable $z$ :

$$
\begin{array}{ll}
\min _{x_{i} \in \mathbb{R}^{n}} & \sum_{i=1}^{N} f_{i}\left(x_{i}\right) \\
\text { s.t. } & x_{i}-z=0, \forall i .
\end{array}
$$

This is called the global consensus problem, since the constraint is that all the local variables should agree, i.e., be equal. ADMM for problem (A.6) can be derived directly from the augmented Lagrangian:

$$
\begin{array}{r}
\mathfrak{L}_{\rho}\left(x_{1}, \ldots x_{N}, y, z\right)= \\
\sum_{i}^{N} f_{i}\left(x_{i}\right)+\left(y_{i}\right)^{T}\left(x_{i}-z\right)+\frac{\rho}{2}\left\|x_{i}-z\right\|_{2}^{2} .
\end{array}
$$


The resulting ADMM algorithm is the following:

$$
\begin{gathered}
x_{i}^{[t+1]}:= \\
\arg \min \left\{f_{i}\left(x_{i}\right)+\left(y_{i}\right)^{T}\left(x_{i}-z\right)+\frac{\rho}{2}\left\|x_{i}-z\right\|_{2}^{2}\right\} . \\
z^{[t+1]}:=\arg \min \sum_{i}^{I}\left\{\left(y_{i}\right)^{T}\left(x_{i}-z\right)+\frac{\rho}{2}\left\|x_{i}-z\right\|_{2}^{2}\right\} . \\
y^{[t+1]}=y^{[t]}+\rho\left(x_{i}^{[t+1]}-z^{[t+1]}\right) .
\end{gathered}
$$

The first and last steps are carried out independently for each $i=1, \ldots, N$. In the literature, the processing element that handles the global variable $z$ is sometimes called the central collector or the fusion center. The dual variables are separately updated to drive the variables into consensus, and quadratic regularization helps pull the variables toward their average value while still attempting to minimize each local $f_{i}$. We can interpret consensus ADMM as a method for solving problems in which the objective and constraints are distributed across multiple processors. Each processor only has to handle its own objective and constraint term, plus a quadratic term which is updated each iteration. The quadratic terms (or more accurately, the linear parts of the quadratic terms) are updated in such a way that the variables converge to a common value, which is the solution of the full problem. 


\section{Appendix B}

\section{Computational Complexity Analysis}

The complexity of Alg. 4.2 can be evaluated as follows. Firstly, the number of variables is $I J$ and the number of dual variables is $(I+1) J$, thus the elementary

steps needed for calculating $c_{i j}^{[t+1]}, \mu_{j}^{[t+1]}$ and $\nu_{i j}^{[t+1]}$ are $(2 I+1) J$. Secondly, the maximum loops of iterations for the calculation are $T$ loops. Therefore, as other steps in Alg. 4.2 is independent from $I$ and $J$, Alg. 4.2 runs in polynomial time with the time complexity of $\mathcal{O}(T(2 I+1) J)=\mathcal{O}(I J)$. Similarly, if Alg. 4.2 is running in a distributed manner at each BS. The number of variables and dual variables at each BS are at most $I$ and $(1+I)$ respectively. Thus, the elementary steps for calculation are $(2 I+1)$, which means the time complexity of each BS is $\mathcal{O}(I)$.

The complexity for solving the problem (4.15) can be evaluated similarly to the above if we use dual methods (e.g., primal-dual interior method). The total number of variables is $Q I+I J+J^{2}$ and the number of dual variables is $J^{2}+J+I+1+I+Q I$. As a result, the elementary steps needed for calculating those variables at most are $T^{\prime}\left(2 Q I+2 J^{2}+I J+2 I+J+1\right)$ where $T^{\prime}$ is them maximum iterations, which means the time complexity for solving problem (4.15) is $\mathcal{O}(\max \{I, J\} J$ ) (a polynomial time algorithm).

In Alg. 4.3, the number of bandwidth prises is $J$, which means $J$ steps are needed 
to update $\lambda_{j}$. As we explained above, the time complexity for updating $r_{l j}^{f,[k+1]}, r_{i j}^{a,[k+1]}$, and $x_{i q}^{[k+1]}$ are $\mathcal{O}(\max \{I, J\} J)$ and for $c_{i j}^{[t+1]}$ are $\mathcal{O}(I J)$, respectively. Consequently, Alg. 4.3 obtains the solution with the the time complexity of $\mathcal{O}(\max \{I, J\} J)$ as the complexities of rounding $\left\{c_{i j}\right\}$ and $\left\{x_{i q}\right\}$ up are just $\mathcal{O}(I J)$ and $\mathcal{O}(Q I)$ (usually $Q \ll J)$. 